RIKEN-TH-70

hep-th/0604211

\title{
Improved Perturbation Method and its Application to the IIB Matrix Model
}

\author{
T. Aoyama and Y. Shibusa \\ Theoretical Physics Laboratory, RIKEN, Wako, 351-0198, Japan
}

\begin{abstract}
We present a new scheme for extracting approximate values in "the improved perturbation method", which is a sort of resummation technique capable of evaluating a series outside the radius of convergence. We employ the distribution profile of the series that is weighted by $n$ th-order derivatives with respect to the artificially introduced parameters. By those weightings the distribution becomes more sensitive to the "plateau" structure in which the consistency condition of the method is satisfied. The scheme works effectively even in such cases that the system involves many parameters. We also propose that this scheme has to be applied to each observable separately and be analyzed comprehensively.

We apply this scheme to the analysis of the IIB matrix model by the improved perturbation method obtained up to eighth order of perturbation in the former works. We consider here the possibility of spontaneous breakdown of Lorentz symmetry, and evaluate the free energy and the anisotropy of space-time extent. In the present analysis, we find an $\mathrm{SO}(10)$-symmetric vacuum besides the $\mathrm{SO}(4)$ - and $\mathrm{SO}(7)$-symmetric vacua that have been observed. It is also found that there are two distinct $\mathrm{SO}(4)$-symmetric vacua that have almost the same value of free energy but the extent of space-time is different. From the approximate values of free energy, we conclude that the $\mathrm{SO}(4)$-symmetric vacua are most preferred among those three types of vacua.

PACS numbers: $02.30 . \mathrm{Mv}, 11.25 .-\mathrm{w}, 11.25 . \mathrm{Yb}, 11.30 . \mathrm{Cp}, 11.30 . \mathrm{Qc}$
\end{abstract}




\section{INTRODUCTION}

String theory is the unique theory that contains massless spin-two particles, i.e. gravitons [1], and thus it is considered to provide an unified microscopic description of the universe including gravitational interactions. For this reason the string theory has been subjected to intensive studies. However, it is recognized that the perturbative string theory fails to single out our universe as the unique vacuum of the theory [2]. Therefore we are forced to pursue non-perturbative formulations. The IIB matrix model (also called the IKKT matrix model) is proposed as a constructive formulation of the superstring theory [3, 4]].

A significant feature of the IIB matrix model is that the space-time itself is expressed by the eigenvalue distribution of 10 bosonic matrices, and thus it is treated as a dynamical variable of the model. The origin of our four-dimensional space-time can be argued in the context of the IIB matrix model as a spontaneous breakdown of Lorentz symmetry. In this regard, we have to understand non-perturbative properties of the model.

The mechanism of the spontaneous breakdown of Lorentz symmetry in reduced matrix models has been examined in various approaches. It has been recognized from those works that the fermionic part of the action plays a crucial role [5, 6, 7, $8,9,10,11]$.

Unveiling dynamical aspects of the model is, in general, quite a difficult problem. The MonteCarlo method is a powerful tool for exploring such non-perturbative properties of a model. However, it is not applicable (or at least quite difficult to apply) to the IIB matrix model due to complex phase of the action derived from the fermionic part ${ }^{1}$. The improved perturbation method (also called the Gaussian expansion method) is an alternative approach. It is considered as a sort of variational method [13, 14, 15, 16, 17, 18]. It has been successfully applied to various models [19, 20, 21, 22], and applications to matrix models were done in Refs. [23, 24, 25].

The application to the IIB matrix model was first achieved in Ref. [26] in which various patterns of symmetry breaking that preserve $\mathrm{SO}(d)$ subgroup of the original ten-dimensional rotational symmetry (ansatz) were examined, and as a conclusion, four-dimensional universe is the most preferred among them based on the comparison of free energy. The Gaussian expansion method was reformulated as an improvement of perturbative series expansion in Ref. [27]. The improved Taylor expansion (ITE), as it is referred, opened a way toward more general applications that

\footnotetext{
${ }^{1}$ A novel technique called the factorization method is proposed to resolve the complex action problem in the Monte Carlo simulations [11, 12].
} 
incorporate quadratic and other types of interactions. The ITE prescription was employed for the IIB matrix model up to fifth order of perturbation in Ref. [27]. It has been proceeded to even higher orders and extended ansatz [28, 29, 30]. The mechanism of the spontaneous symmetry breakdown is further examined in a simplified model via the Gaussian expansion method in Ref. [31].

In the present paper, we investigate the non-perturbative solutions of the IIB matrix model by this technique (which we call "the improved perturbation method" here). We focus on the possibility that the original $\mathrm{SO}(10)$ symmetry of the IIB matrix model may be spontaneously broken to result in our universe which spreads in four directions and has $\mathrm{SO}(4)$ rotational symmetry ${ }^{2}$. In such cases it is important to see that the method is applicable to the models that exhibit phase transitions. In the former work [22], we applied this method to the Ising model and found that the improved perturbation method extracts the information of the ordered phase from an expansion about the vacuum in the disordered phase. It is also observed that an unstable vacuum is identified even though it has larger value of free energy than that of the stable vacuum. We expect that the improved perturbation method reveals different patterns of symmetry breaking that may be developed as unstable or metastable vacua.

The improved perturbation method is considered as a sort of resummation of perturbative series by introducing the artificial parameters into the model. The approximate value of the series is obtained by evaluating it in the region of the artificial parameter space that realizes the principle of minimal sensitivity [16]. We call such a region as "plateau", in which the dependence on those parameters would vanish effectively and the exact value would be reproduced. It works as a consistency condition for the parameters.

The concept of plateau is rather obscure, and there is not yet any proper treatment of mathematical rigor. We need a practical scheme for extracting the approximate value of the series. In addition, it should be free from the ambiguity due to the subjectivity of recognition as much as possible. In the former works, mainly two approaches have been propounded. One is to take the values at the extrema of the improved series with respect to the artificial parameters as approximate values [26]. The other is to take the values which corresponds to the mode of distribution of the evaluated values around the accumulation of extrema. The latter is called the histogram technique [32, 33].

In this article we propose a new prescription which enables us to extract a good approxima-

\footnotetext{
${ }^{2}$ We perform the Wick rotation to the IIB matrix model and discuss in Euclidean space-time.
} 
tion from the improved series. It is achieved by incorporating the appropriate weightings in the histograms that are given by the inverse of derivatives. This method will be applicable to various models which have many parameters.

It has also been customary to evaluate the various observables based on the information of plateau of the free energy. On extracting the approximate value of any observable, the information of minimal sensitivity of only improved free energy is taken into account. To be more precise, one takes the value of improved perturbative series of an observable at extrema of improved free energy with respect to the artificial parameters as approximate values of that observable. We insist that the improved perturbation method has to be applied independently for each observable of interest.

We apply the new scheme to the analysis of the IIB matrix model by means of the improved perturbation method. It is based on the improved series that is obtained in former works up to eighth order of perturbation for $\mathrm{SO}(d)$-preserving configurations as ansatz ( $d=4$ and 7) [29].

This paper is organized as follows. In Section $\amalg$ we provide a description of the resummation technique called the improved perturbation method, including a review of the method. In Section III, we propose a new prescription which extracts the approximate value from improved perturbative series. In Section IV, we apply the improved perturbation to the IIB matrix model and survey the non-perturbative solutions with various patterns of symmetry breaking. Section $\nabla$ is devoted to the conclusion. In Appendix A, we present a detail of analysis performed in Section IV

\section{IMPROVED PERTURBATION}

It is generally believed that the perturbative expansion (also including $\frac{1}{N}$-expansion and $\varepsilon$ expansion) is asymptotic, and diverges beyond some finite orders. Nevertheless, what we can evaluate for most of the theories are only those series expansions, from which we have to draw physical information of the theory. Therefore, we need a method to estimate the exact value from the series with the parameter (coupling constants, and so on) outside the original convergence radius. For this purpose we introduce here a method which has been successfully applied to the IIB matrix model and other general models. 


\section{A. Prescription}

We implicitly use $\lambda$ as coupling constant and $m$ as parameters of the model collectively such as masses. Let us assume that the observables of a theory would be exactly described by a function $F(\lambda, m)$. Perturbation theory provides an expansion of $F$ as a power series of $\lambda$ about $\lambda=0$, with $n$th coefficient denoted as $f_{n}(m)$ :

$$
F(\lambda, m)=\sum_{n=0}^{\infty} \lambda^{n} f_{n}(m) .
$$

In the actual cases, we only have finite portion of the series up to order $N$,

$$
F^{N}(\lambda, m)=\sum_{n=0}^{N} \lambda^{n} f_{n}(m) .
$$

The question is whether we can presume the exact value $F(\lambda, m)$ at the given parameters $\lambda$ and $m$ from the series $F^{N}$ above. In many cases the convergence radius for $\lambda$ is zero, so we can not expect that $F^{N}$ gives a reliable approximation.

Now we consider a modification of the series along the following prescriptions. First we perform a shift of parameters:

$$
\begin{aligned}
& \lambda \longrightarrow g^{p} \lambda, \\
& m \longrightarrow m_{0}+g^{q}\left(m-m_{0}\right),
\end{aligned}
$$

where we have introduced $g$ as a formal expansion parameter, and $m_{0}$ as a set of artificial parameters. $p$ and $q$ are taken arbitrarily. We deform the series by the substitution (3), and then we reorganize the series in terms of $g$, drop the $O\left(g^{M+1}\right)$ terms, and finally set $g$ to 1 . We obtain the improved perturbative series $\widetilde{F}^{N}$ as

$$
F^{N}(\lambda, m) \longrightarrow \widetilde{F}^{N}\left(\lambda, m ; m_{0}\right)=\left.F^{N}\left(g^{p} \lambda, m_{0}+g^{q}\left(m-m_{0}\right)\right)\right|_{g^{M}, g \rightarrow 1}
$$

Here, we adopt a notation $\left.\right|_{g^{M}, g \rightarrow 1}$ to represent the operation that we disregard the $O\left(g^{M+1}\right)$ terms and then put $g$ to 1 .

It should be noted that by simply setting $g=1$, the modification itself becomes trivial and the series would be independent of the parameters $m_{0}$. However, due to dropping the $O\left(g^{M+1}\right)$ terms, the deformed series does depend on $m_{0}$.

To turn the argument around, we adopt here the principle of minimal sensitivity that the exact value $F$ will be reproduced when the improved series depends least on the artificial parameters $m_{0}$. 
It provides a sort of consistency condition on $m_{0}$; by tuning the parameters to the solution of the condition, we will have a good approximation of $F$.

In the above we have introduced the arbitrary parameters $p, q, M$. If the region emerges in the parameter space of $m_{0}$ that realizes minimal sensitivity, the approximate value must be independent of the choice of parameters $p, q, M$. However, with the limited orders of perturbation, the signal of minimal sensitivity is often weak in actual cases. So we have to find the optimal values of parameters $p, q, M$ in order to make the signal clear. We have to keep in mind that large values of $p$ and $q$ turn to throw away the information of higher order terms of perturbative series, and taking larger value of $M$ than that of $N$ is meaningless. Therefore, we set the parameters as $p=q=1$ and $M=N$ throughout this paper.

\section{B. Example}

For example, we apply the improved perturbation method to a simple 1-dimensional function,

$$
F(\lambda, m)=\frac{1}{1+\lambda m}
$$

and try to estimate $F(\lambda, m)$ at $\lambda=1$ and $m=3 / 2$.

The Taylor expansion about $\lambda=0$ up to order $N$ gives the following series:

$$
F^{N}(\lambda, m)=\sum_{n=0}^{N}(-1)^{n} \lambda^{n} m^{n}
$$

This series at $N \rightarrow \infty$ has finite convergence radius $|\lambda m|<1$. The situation is depicted in Fig. 1(a) where $F^{N}$ of various $N$ are shown as a function of $m$ when $\lambda=1$.

Now we deform the series along the prescription in the previous section, to obtain the improved series $\widetilde{F}^{N}$ :

$$
\widetilde{F}^{N}\left(\lambda, m ; m_{0}\right)=\left.\sum_{n=0}^{N}(-1)^{n}(\lambda g)^{n}\left(m_{0}+g\left(m-m_{0}\right)\right)^{n}\right|_{g^{N}, g \rightarrow 1} .
$$

Here, $\left.\right|_{g^{N}, g \rightarrow 1}$ denotes disregard for the $O\left(g^{N+1}\right)$ terms followed by setting $g=1$.

The improved series behaves as shown in Fig. 1(b), when the artificial parameter $m_{0}$ is taken to be 1.1. In this case, the functions $\widetilde{F}^{N}\left(\lambda=1, m ; m_{0}=1.1\right)$ become close to the exact value in the region $m \sim 1.5$, and we will have a good approximation of the original function $F$ at $m=3 / 2$.

To find the optimum of parameter $m_{0}$, we consider $\widetilde{F}^{N}\left(\lambda, m ; m_{0}\right)$ as a function of $m_{0}$ with $m$ and other original parameters fixed to the specified values, (e.g. $\lambda=1$ and $m=3 / 2$ in this case). 


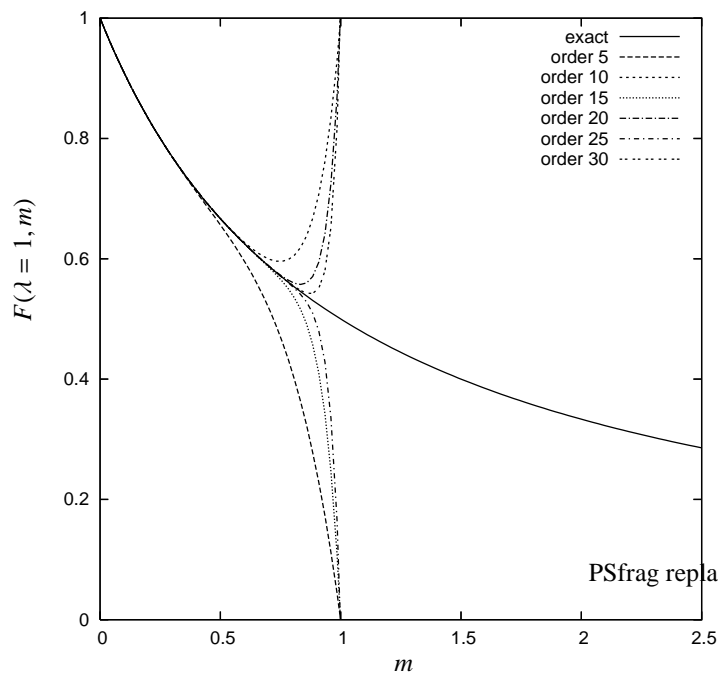

(a)

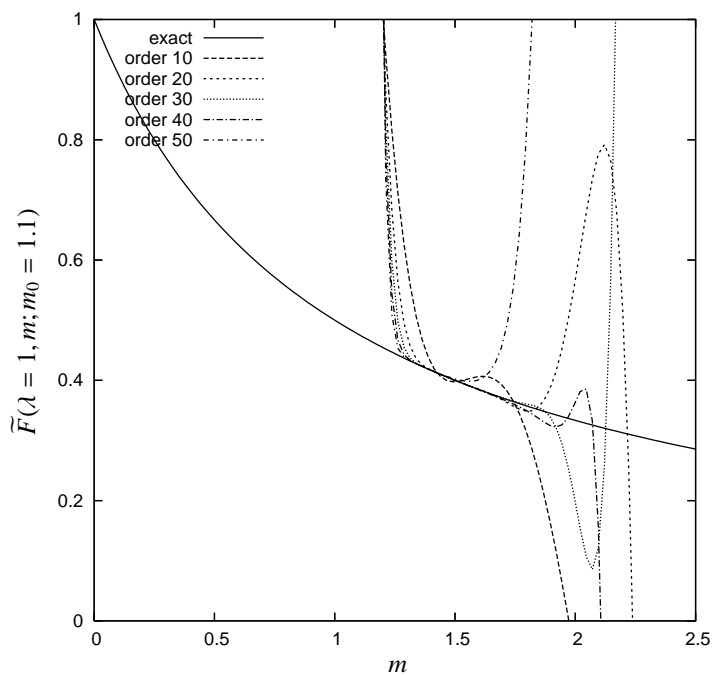

(b)

FIG. 1: (a) Taylor series of $F(\lambda, m)=\frac{1}{1+\lambda m}$ and (b) improved series at $m_{0}=1.1$.

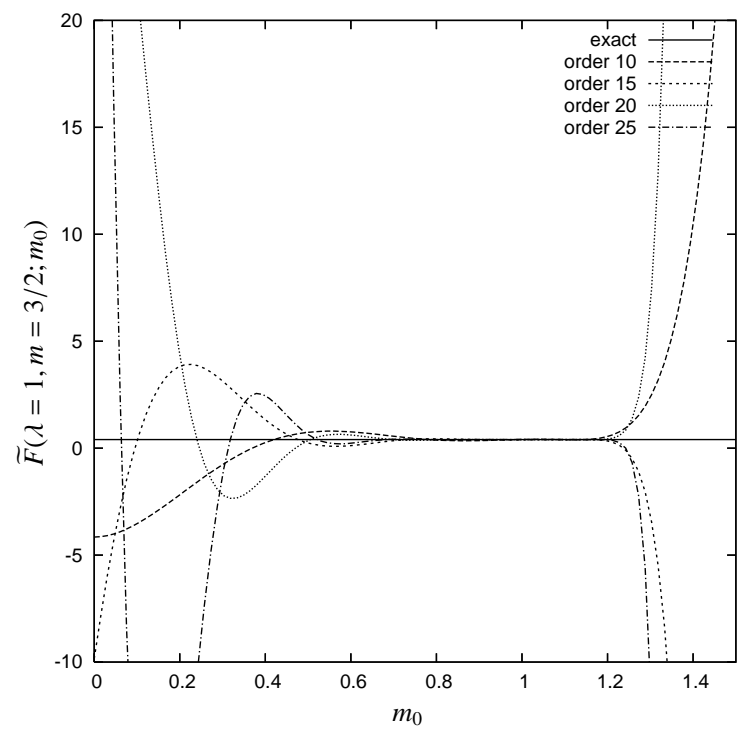

FIG. 2: Improved series as a function of artificial parameter $m_{0}$.

It can be seen that in some region of parameter $m_{0}=0.7 \sim 1.1, \widetilde{F}^{N}$ stays stable and gives good approximation to the exact value, $F=0.4$ (Fig. 2). We call such a stable region as "plateau", where the principle of minimal sensitivity is realized and the exact value will be reproduced. 


\section{Properties of improved perturbative series}

\section{Details}

We will elucidate the concrete prescription of the improved perturbation method.

The original perturbative series $F^{N}(\lambda, m)$ up to order $N$ is given in the following expression, where $f_{n}(m)$ is the $n$th order coefficient:

$$
F^{N}(\lambda, m)=\sum_{n=0}^{N} \lambda^{n} f_{n}(m)
$$

By the shift of the parameters,

$$
\begin{aligned}
& \lambda \longrightarrow g \lambda, \\
& m \longrightarrow m_{0}+g\left(m-m_{0}\right),
\end{aligned}
$$

$F^{N}$ is deformed as,

$$
\begin{aligned}
F^{N}(\lambda, m) & \longrightarrow \sum_{n=0}^{N}(g \lambda)^{n} f_{n}\left(m_{0}+g\left(m-m_{0}\right)\right) \\
& =\sum_{k=0}^{\infty} g^{k} \sum_{n=0}^{\min (N, k)} \lambda^{n} \frac{1}{(k-n) !}\left(m-m_{0}\right)^{k-n} f_{n}^{(k-n)}\left(m_{0}\right),
\end{aligned}
$$

Here, each coefficient $f_{n}$ is expanded in Taylor series about $m=m_{0}$, with $f_{n}^{(k)}$ as $k$ th derivative of $f_{n}(m)$ with respect to $m$. The series is then reorganized in powers of $g$. Then we drop the $O\left(g^{N+1}\right)$ terms, and finally set $g$ to 1 . As a result, we will have the improved series $\widetilde{F}^{N}$ :

$$
\widetilde{F}^{N}=\sum_{n=0}^{N} \lambda^{n} \sum_{k=0}^{N-n} \frac{1}{k !}\left(m-m_{0}\right)^{k} f_{n}^{(k)}\left(m_{0}\right) .
$$

It turns out that the improved perturbation method replaces each coefficient of the original series, $f_{n}(m)$, by its Taylor expansion about a shifted point $m_{0}$,

$$
f_{n}(m) \longrightarrow \widetilde{f}_{n}\left(m ; m_{0}\right)=\sum_{k=0}^{N-n} \frac{1}{k !}\left(m-m_{0}\right)^{k} f_{n}^{(k)}\left(m_{0}\right)
$$

It should also be noted that the expansion in eq. (12) is taken only up to $(N-n)$ th order. This feature works to suppress the large fluctuations coming from higher order coefficients, which have caused divergent behavior of the original series. Thus we could expect better estimates by this improved perturbation method.

The important point is that the reorganized series should be truncated at finite order $N$. If we kept all the terms and if we could switch the order of two summations in eq. (10), the procedure should become trivial, only to obtain the original series. 


\section{Convergence}

The convergence property of the improved series with respect to $\lambda$ is, however, not affected significantly. The ratio of $(n+1)$ th coefficient against $n$th one,

$$
\frac{\widetilde{f}_{n+1}}{\widetilde{f_{n}}}=\frac{\sum_{k=0}^{N-n-1} \frac{1}{k !}\left(m-m_{0}\right)^{k} f_{n+1}^{(k)}\left(m_{0}\right)}{\sum_{k=0}^{N-n} \frac{1}{k !}\left(m-m_{0}\right)^{k} f_{n}^{(k)}\left(m_{0}\right)}
$$

has no particular structures such as singularities, at least in obvious manner. There, the parameters $m_{0}$ are determined according to the following argument; minimal sensitivity condition will be realized ideally when $p$ th or lower derivatives of $\widetilde{F}^{N}$ with respect to the parameters $m_{0}$ become zero:

$$
\begin{aligned}
0 & =\frac{d^{p}}{d m_{0}^{p}} \widetilde{F}^{N}\left(\lambda, m ; m_{0}\right) \\
& =\sum_{n=0}^{N} \lambda^{n} \sum_{q=0}^{p-1}\left(\begin{array}{c}
p-1 \\
q
\end{array}\right)(-)^{q} \frac{1}{(N-n-q) !}\left(m-m_{0}\right)^{N-n-q} f_{n}^{(N-n+p-q-1)}\left(m_{0}\right) .
\end{aligned}
$$

We will examine the condition further in later sections.

\section{Limitations}

As mentioned before, the improved perturbation method does not much alter the convergence properties. There are some class of functions such that the improved series actually is convergent in the "plateau" region, and the procedure of improved perturbation works as a sort of analytical continuation. For example, the function $\frac{1}{1+\lambda m}$ discussed in the previous subsection belongs to this class[34]. However, most of the series that appear in physics do not have the property like this.

If the original series is asymptotic or is evaluated at outside of convergence region, the improved series may also show the divergent behavior at excessively high orders. We will illustrate this feature by a simple example. Consider an ordinary function $F$,

$$
F(\lambda, m)=\frac{1}{1+\lambda m}+\frac{1}{1+\lambda m+(\lambda m)^{3}},
$$

and generate a Taylor series about $\lambda=0$ up to $\operatorname{order} N$. The radius of convergence of the original series is $(\lambda m) \sim 0.7$. Then, we apply the improved perturbation method to this series. As shown in Fig. 3(a), the improved series at $\lambda=1, m=3 / 2$ fluctuates violently with respect to $m_{0}$. The deviation from the exact value grows larger as the order increases. (The artificial parameter $m_{0}$ is 


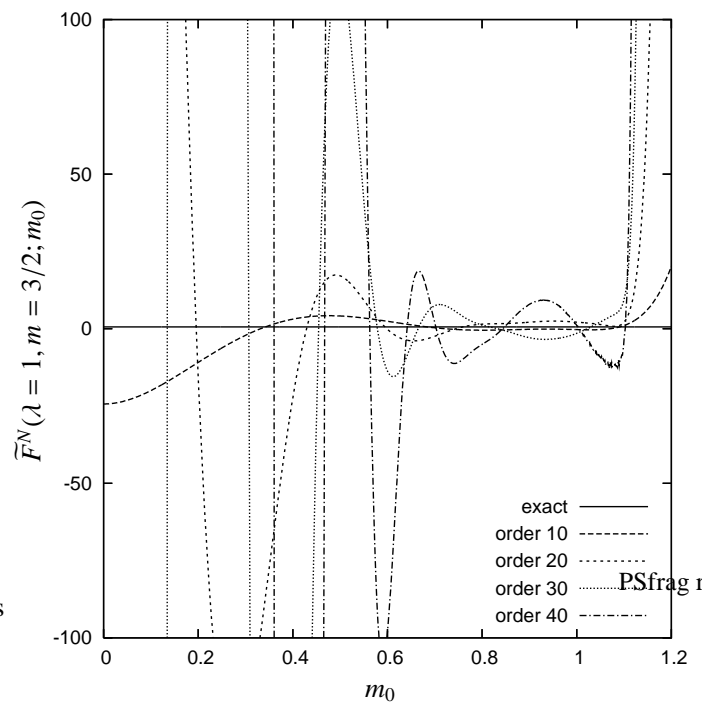

(a)

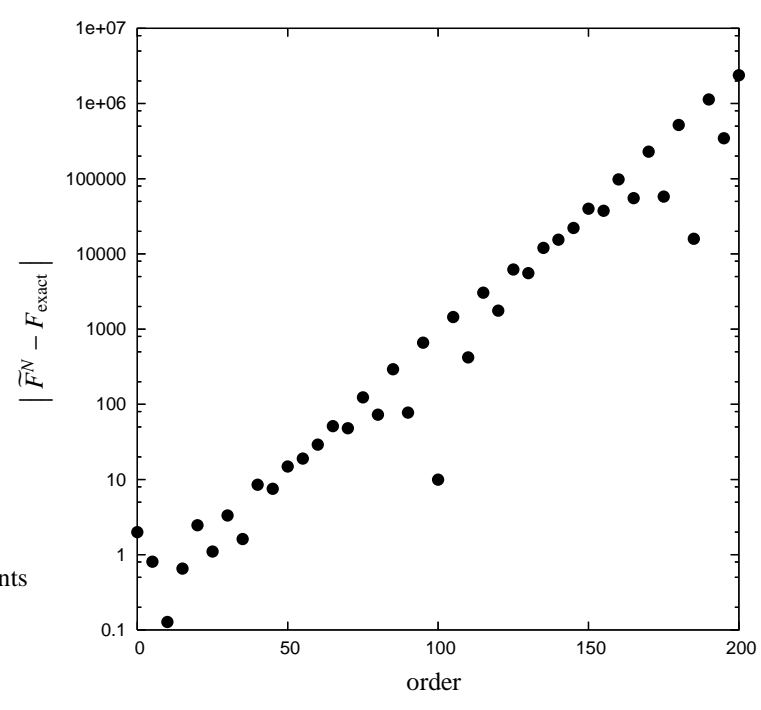

(b)

FIG. 3: (a) Large fluctuation of $\tilde{F}$ as a function of $m_{0}$. (b) Deviation between $\tilde{F}^{N}$ and the exact value. $\left(m_{0}\right.$ is chosen to be 0.95 .)

chosen to be $m_{0}=0.95$.) The improved perturbation method fails to reproduce the exact result in this case at high enough orders (Fig. 3(b)).

In order to obtain a reliable estimate by the improved perturbation method, we have to combine various information of the series of different orders, and examine synthetically the whole profile of the series.

\section{4. $\quad$ Ring properties}

In this section, we examine how the improved perturbation method affects the function ring $C^{\infty}$. We are especially interested in the derivative operation, for the expectation value of an operator is related to the derivative of the free energy with the corresponding source term.

Usually we can introduce an equivalence relation into a set of elements of function ring, and classify them into equivalence classes. Now we insist that the plateau criteria works for such classification; two elements of the function ring are equivalent if there exists intersection between "plateau" of each function where minimal sensitivity is realized.

First we consider the add operation. Assume that we have two functions, $F$ and $G$, and the improved series, $\widetilde{F}^{N}$ and $\widetilde{G}^{N}$, respectively. The procedure to obtain the improved series is basically Taylor expansion, and thus linear. If $\widetilde{F}^{N}$ and $\widetilde{G}^{N}$ have intersecting region where minimal 
sensitivity condition for each series is realized simultaneously, then the improved series of sum of the functions, $(\widetilde{F+G})^{N}$ also stays stable and reproduces the exact value there.

On the other hand, when $\widetilde{F}^{N}$ and $\widetilde{G}^{N}$ do not have overlapped parameter region, the sum $(\widetilde{F+G})^{N}$ does not in general bear stable region in their plateaux.

Thus, the improved perturbation method preserves the add operation among the elements of function ring that belong to the same equivalence class based on the presence of intersection of "plateau".

Unlike the case of the add operation, there is no reliable discussion on the product operation of function ring. However, from several examples, it seems that the procedure also preserves the product operation among the elements of the same equivalence class.

Next we consider the derivative operation. Similar to the ordinary function, the derivative of the improved series, $\widetilde{F}^{N}\left(\lambda, m ; m_{0}\right)$, with respect to $m$ will be defined as follows:

$$
\frac{d}{d m} \widetilde{F}^{N}\left(\lambda, m ; m_{0}\right)=\lim _{\epsilon \rightarrow 0} \frac{\widetilde{F}^{N}\left(\lambda, m+\epsilon ; m_{0}\right)-\widetilde{F}^{N}\left(\lambda, m ; m_{0}\right)}{\epsilon} .
$$

We will designate as $\mathrm{R}(\lambda, m)$ the region in the parameter space $m_{0}$ where the minimal sensitivity condition is satisfied. We also call it as "region of minimal sensitivity" below.

If two regions of minimal sensitivity for physical parameters at $m+\epsilon$ and $m$ have overlap,

$$
\mathrm{R}(\lambda, m+\epsilon) \cap \mathrm{R}(\lambda, m) \neq \emptyset,
$$

we would conclude from the argument for the add operation, that the derivative function defined in eq. (16) bears the region of minimal sensitivity in the intersection of the two regions. In short, for a function and its derivative, the region of minimal sensitivity will be expected to emerge somehow close to each other.

Otherwise, it may happen that the region of minimal sensitivity of $\widetilde{F}^{N}$ and its derivatives are uncorrelated. Then, in what situation does the overlap vanish, i.e. $\mathrm{R}(\lambda, m+\epsilon) \cap \mathrm{R}(\lambda, m)=\emptyset$ ? This question is deeply related to the critical behavior of the underlying physical model. In usual cases, free energy and various expectation values are regular with respect to moduli parameters, and the region $\mathrm{R}(\lambda, m)$ evolves regularly as well. However, at singular points of moduli space such as those where the symmetry of the model is enhanced, $\mathrm{R}(\lambda, m)$ should emerge in a quite different manner; thus even for infinitesimally small deviation of moduli parameter, $\mathrm{R}(\lambda, m)$ and $\mathrm{R}(\lambda, m+\epsilon)$ may not have overlaps.

Such a situation often occurs in the physical models of our interests. For example, in Ising model, the region of minimal sensitivity drastically changes between high temperature phase and 
low temperature phase. In the IIB matrix model (which will be examined extensively in later sections), we are interested in the massless region, where the parameters become singular. In these cases, the free energy and the expectation values of various observables show different behavior, and therefore we have to treat them separately.

\section{PLATEAU CONDITIONS}

In this section, we will elucidate the criteria how to determine desirable regions of artificial parameters, and to estimate the approximate value from the improved series. A guiding principle, minimal sensitivity, will be realized in regions in the parameter space where the improved function stays rather stable. We call such region as plateau.

The subject of this section is to develop a concrete and objective scheme for identifying plateau. Besides, the notion, "stable" is obscure, and so the procedure may also provide an inductive definition of plateau itself.

\section{A. Minimal sensitivity and plateau}

The improved perturbation method introduces artificial parameters, $m_{0}$, which we need to determine by some means. Here we adopt as a guiding principle, minimal sensitivity, that the improved function should depend least on those parameters; this is because $m_{0}$ were originally introduced as the nominal shift of parameters, though the dependence on them appeared due to dropping the $O\left(g^{N+1}\right)$ terms. If there exists a region in the parameter space $m_{0}$ where the dependence on $m_{0}$ vanishes effectively, the exact value should be reproduced there. In the following we denote simply by $\widetilde{F}^{N}$ the improved series $\widetilde{F}^{N}\left(\lambda=\lambda_{*}, m=m_{*} ; m_{0}\right)$ where we substitute each desirable values $\lambda_{*}$ and $m_{*}$ for $\lambda$ and $m$. This series is function of artificial parameters $m_{0}$.

The principle of minimal sensitivity is realized through the emergence of the region in the parameter space where the improved series stays stable against any variation of artificial parameters; we call such a region as "plateau". What we have to do for determining $m_{0}$ is to identify where the improved series becomes stable.

When the number of parameters are one (or at most two), the stable region of the function will be pointed out by just drawing a graph of it. However, the visualization will not be possible for the cases with more than two parameters. Moreover, even though it is possible, it may sometimes 
lead to misjudgment by changing the scale arbitrarily. Therefore we need a concrete scheme for identifying the plateau applicable to multi-parameter space, which is not affected by intuitive guesses.

\section{B. Identifying plateau}

The ideal realization of the principle of minimal sensitivity may have such a property that the improved series is totally independent of the artificial parameters $m_{0}$ in a region. It involves the situation that all orders of derivatives of the improved series with respect to $m_{0}$ are zero in the region. However, such an ideal "plateau" is not realized in the actual cases because we have only finite order of series. Typical profile of the improved series that forms plateau exhibits a flat region in which the series fluctuates bit by bit; it would accompany a number of local maxima and minima there. Thus, to turn the argument around, we consider the accumulations of extrema as indications of plateau (or its candidates).

It should be noted that there is also a case when the series becomes stable without forming any extrema in that region; we may miss such plateau by the above speculation. We will discuss this type of plateau in later section.

\section{Cluster identification}

Assume that we have already found extrema of the improved series in the (multi-dimensional) parameter space. Next issue is to identify the accumulation among them. We denote the coordinate of $i$ th extrema by $\vec{x}_{i}$, and the distance between $i$ th and $j$ th extrema by $d_{i j}$. The definition of distance in the parameter space will be presented later.

The distribution $\rho(r)$ of the distances $d_{i j}$ shows characteristic behavior according to the presence of clusters of points. Here, $\rho(r)$ is given by the number of pairs $(i, j)$ whose distance $d_{i j}$ falls on to $r<d_{i j}<r+\delta r$. As depicted in Fig. 4, when one or more clusters of points exist, $\rho(r)$ has a large peak near $r=0$ and some small bumps at larger $r$. Let $n$ the number of points in the cluster. Then the peak height is proportional to $n^{2}$, while those of other bumps are of order $n$. If there are no such clusters and the points are scattered rather uniformly, $\rho(r)$ shows continuous distribution. This concept can be extended straightforwardly to the case in which more than one cluster are formed. From those distinctive properties, we are able to identify the formation of clusters and the 

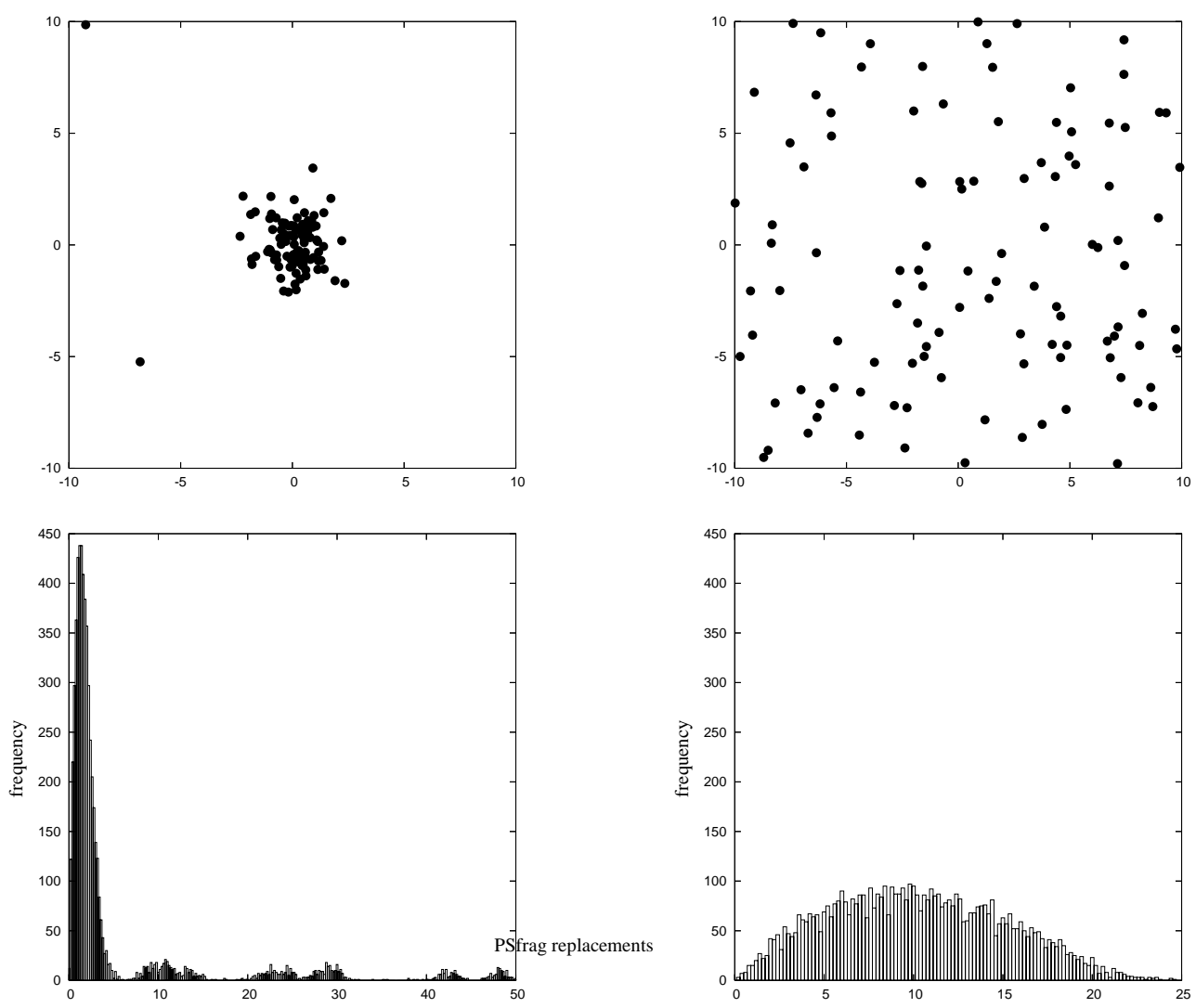

(a)

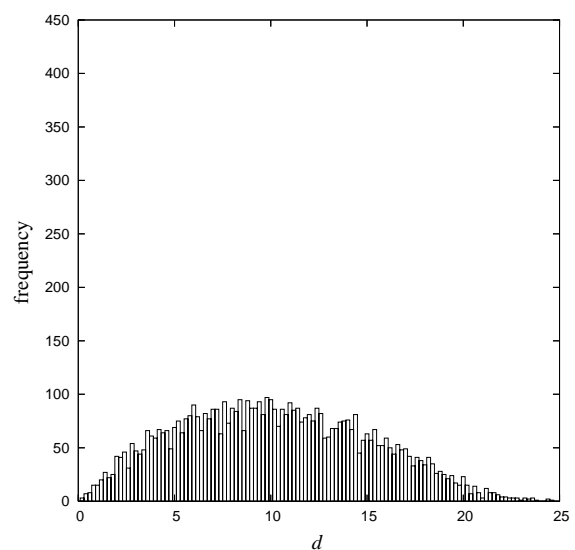

(b)

FIG. 4: Distributed points (above) and distributions of distance between points (below) for each case: (a) forming the cluster, and (b) straggling uniformly.

set of points belonging to them.

There is no intrinsic definition of distance $d_{i j}$ in multi-dimensional parameter space. Therefore we have to choose a suitable definition. It introduces metric space and must obey the triangle inequality.

A naïve choice is as follows:

$$
d_{i j}^{2}=\left|\vec{x}_{i}-\vec{x}_{j}\right|^{2}
$$

As another choice, if we can define a reasonable metric $g_{a b}$ from some argument such as dimensional analysis, we will have,

$$
d_{i j}=\int_{\text {geodesic }} \sqrt{-g} d s,
$$

along geodesic between $\vec{x}_{i}$ and $\vec{x}_{j}$. The geodesic equation may not be solved nor have any solution; 
we would alternatively choose a linear interpolation with weights by metric as,

$$
d_{i j}=\sum_{k} \sqrt{-g\left(t_{k}\right)}\left|\vec{x}\left(t_{k}\right)-\vec{x}\left(t_{k+1}\right)\right|, \quad \vec{x}\left(t_{k}\right)=\vec{x}_{i}+\frac{k}{N}\left(\vec{x}_{j}-\vec{x}_{i}\right),
$$

though this $d_{i j}$ may not satisfy triangle inequality.

It is not always that two extrema which close to each other in the meaning of the above distances belong to one stable region. This is because it is possible that between these extrema improved series varies its value drastically and vicinity of two extrema encounters by chance. In order to avert this situation, we consider one other choice of distance. This choice comes from extending the concept of moduli space. By the definition of distance we intend to express the degree to which two of the extrema of a function $\widetilde{F}^{N}$ resemble each other. Thus we include the deviation of $\widetilde{F}^{N}$ and its derivatives at these two points into the notion of distance:

$$
d_{i j}^{2}=\left|\vec{x}_{i}-\vec{x}_{j}\right|^{2}+\sum_{k} w_{k}\left|\frac{d^{k} \widetilde{F}^{N}}{d x^{k}}\right|_{\vec{x}_{i}}-\left.\left.\frac{d^{k} \widetilde{F}^{N}}{d x^{k}}\right|_{\vec{x}_{j}}\right|^{2} .
$$

Moreover we can also include the transition as orders of perturbation increase:

$$
d_{i j}{ }^{2}=\left|\vec{x}_{i}-\vec{x}_{j}\right|^{2}+\sum_{M, M^{\prime}=0}^{N} \omega_{M M^{\prime}} \sum_{k} w_{k}\left|\frac{d^{k} \widetilde{F}^{M}}{d x^{k}}\right|_{\vec{x}_{i}}-\left.\left.\frac{d^{k} \widetilde{F}^{M^{\prime}}}{d x^{k}}\right|_{\vec{x}_{j}}\right|^{2} .
$$

The relative weights $w_{k}$ and $\omega_{M M^{\prime}}$ above should be chosen according to the models considered.

In general, we can not introduce a particular choice of metric naturally. Then we try some definitions of metric to a model and investigate the improved perturbative series carefully.

\section{Weighted histogram analysis}

If the improved series $\widetilde{F}^{N}$ bears a plateau, the distribution of the improved series in a region enclosing the plateau should form a peak corresponding to the value of $\widetilde{F}^{N}$, which gives an estimate of the function on the plateau.

Therefore, we choose a region (for ease of operation, we usually consider rectangular one) which encloses accumulation of extrema, and evaluate distribution $\rho(F)$ of $\widetilde{F}^{N}$ [32],

$$
\rho(F)=\int_{A} d x 1, \quad A=\left\{x \mid F<\widetilde{F}^{N}(x)<F+\delta F\right\} .
$$

In multi-dimensional parameter case, the extent of the plateau is not always large nor the shape isometric in the parameter space. Then we need a method to extract the estimated value of $\widetilde{F}^{N}$ on plateau effectively even when the region to be examined is taken roughly. 
The plateau is, if ideally realized, characterized by the feature that the first and higher derivatives of $\widetilde{F}^{N}$ should be zero or small. To enhance the contribution from such flat region, we introduce weight function to the distribution as,

$$
\rho(F)=\int_{A} d x w
$$

There may be some choices of the weight function $w$; for example,

$$
\begin{aligned}
& w_{1}=\frac{1}{\left|\sum_{i} \frac{d \widetilde{F}^{N}}{d x_{i}}\right|^{2}}, \\
& w_{2}=\frac{1}{\left|\operatorname{det} \frac{d^{2} \widetilde{F}^{N}}{d x_{i} d x_{j}}\right|^{2}},
\end{aligned}
$$

and so forth. Here we denote the $i$ th component of coordinates by $x_{i}$.

The histogram of weighted distribution shows sharp peak corresponding to the zeros of $k$ th derivatives of the improved series. Even though the region in question is taken roughly, the contribution from the fluctuating part of $\widetilde{F}^{N}$ is well suppressed, and we will have an estimate of $\widetilde{F}^{N}$ on the plateau.

There is also another choice of weight function based on the convergent behavior of the series on the plateau. If the improved series converges to some value on the plateau, it implies that the difference between the improved series of order $N$ and order $N+1$ diminishes. To reflect such speculation, we would better choose a weight function by

$$
w_{3}=\frac{1}{\left|\widetilde{F}^{N+1}-\widetilde{F}^{N}\right|^{2}} .
$$

In general, we combine the above weight functions on a case-by-case basis. In the following investigation into the IIB matrix model, we use the two weight functions $w_{1}(25)$ and $w_{2}(26)$.

\section{Signal and noise}

When the number of parameters are one (or at most two), we can easily recognize the flat region from the graph. However, there is no such simple scheme in multi-parameter case in general. We are trying to identify plateau from the accumulation of the extrema as a clue. The information we have so far for the improved series $\widetilde{F}^{N}$ is just the location of extrema and profile of (weighted) distribution of $\widetilde{F}^{N}$ in some specified regions. The accumulations of extrema provides candidates 


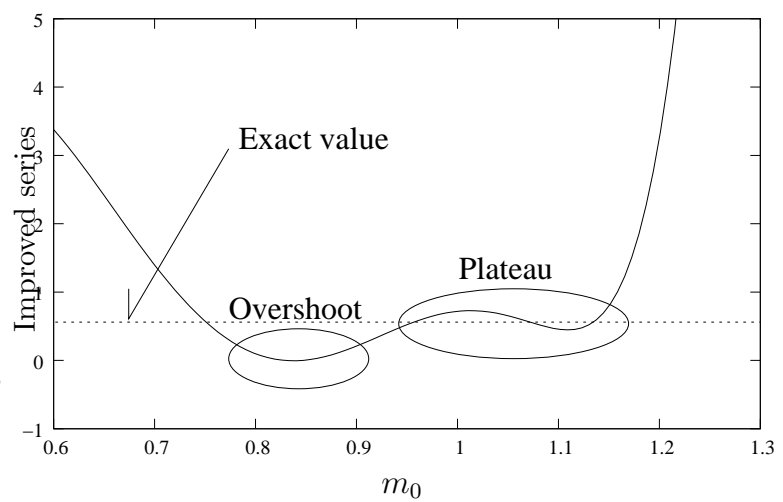

FIG. 5: A characteristic profile of overshoot and plateau.

of the plateau. Among these candidates we have to distinguish the true plateaux, though yet we do not have definite argument on this subject. We exemplify one typical false signal, "overshoot".

It often occurs when a discrete function is approximated by a series of polynomial (consider, for example, Fourier transform of rectangular waves) that the series deviates relevantly at discontinuities, which remains with even higher orders taken into account. Such behavior is called "overshoot". In the current case, the improved series turns to be an approximation of a constant function by a set of polynomials because the exact value is totally independent of artificial parameters. Therefore the improved series are apt to show typical overshoot behavior as well (Fig. 5).

The overshoot is rather isolated from other extrema that belong to a plateau; this should be reflected in the characteristic profile of weighted distributions. The overshoot gives minimum (or maximum) in its neighbor, so the histogram of the distribution weighted by $w_{1}$ shows sharp peak, and it is cut away on lower (or upper) side (Fig. 6(a)). Moreover it often happens that the peaks in the distributions with second and higher derivatives deviate from peaks in that with the first derivative. It is because the overshoot appears as isolated extrema away from those forming flat region. On the contrary, in the case of plateau, the histogram of the distribution weighted by $w_{1}$ and $w_{2}$ shows symmetric shape and a peak of the distribution weighted by $w_{2}$ lies between (or ideally on) peaks of the distribution weighted by $w_{1}$ (Fig. 6(b)). For the reason stated above, a cluster of extrema which corresponds to overshoot can be distinguished from plateau by investigating the shape of distributions.

Besides, it seems that the overshoots of different orders tend to form a line in the parameter space. If there is such sequential structure, we had better suspect that they are overshoots. 


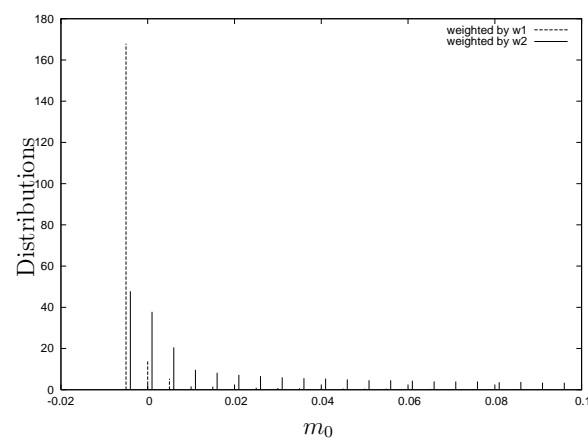

(a)

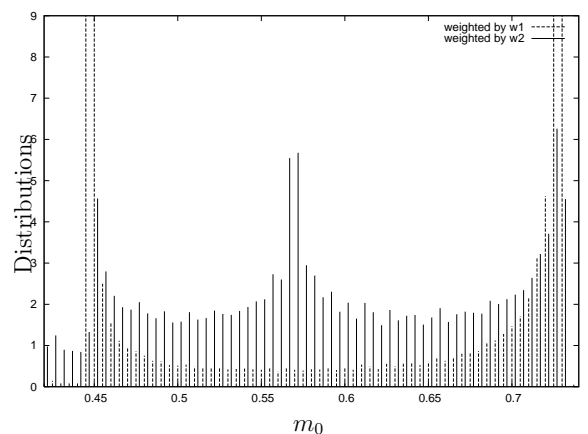

(b)

FIG. 6: (a):Distribution weighted by $w_{1}, w_{2}$ in overshoot region and (b)distribution weighted by $w_{1}, w_{2}$ in plateau region.

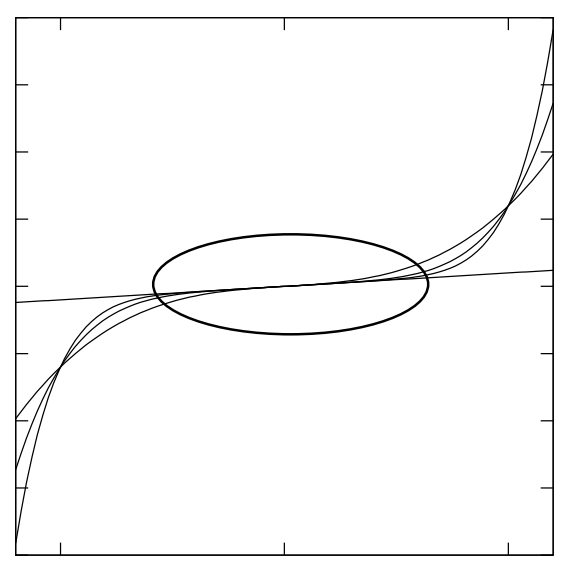

FIG. 7: A conceptual profile of smooth plateau.

\section{Pitfalls and special cases}

\section{Smooth plateau}

In the prescription thus far explained, we assumed that the improved series $\widetilde{F}^{N}$ shows fluctuating behavior on the plateau, accompanied by the accumulation of extrema. If there is a region where $\widetilde{F}^{N}$ becomes stable but varies gently without forming extrema (Fig. 7), it should also be considered as plateau, though the above procedure based on the existence of extrema will not be applicable to such cases. We call this type of plateau as "smooth plateau". However, the histogram will show the peaked structure corresponding to this smooth plateau. The weighted distribution should provide a reasonable estimate of the value. 
The problem is that we do not have any guide to locate the position of plateau in this case. One clue is the consistency with the plateau of other physical quantities. Though the improved series of different observables may show distinctive behavior, the plateau corresponding to the physical states should somehow be realized in each series. We have to guess the region of smooth plateau in parameter space based on those information. If we find a plateau in analysis of an observable, we must suspect the appearance of smooth plateau in neighborhood of that region in the case of the other observables even for absence of any extremum.

It should be noted that we have to take care not to be confused with mere asymptotic behavior of the series. A concrete example will be shown in the analysis of the IIB matrix model in later sections.

\section{Plateau on special hypersurface}

Consider a hypersurface in the parameter space where one of the parameters is zero. If we change the coordinate from $m_{0}$ to $x_{0}=\frac{1}{m_{0}}$, the first derivative with respect to the new coordinate becomes,

$$
\frac{d \widetilde{F}^{N}}{d x_{0}}=-m_{0}{ }^{2} \frac{d \widetilde{F}^{N}}{d m_{0}} .
$$

Even though $\frac{d \widetilde{F}^{N}}{d m_{0}}$ does not vanish, $\frac{d \widetilde{F}^{N}}{d x_{0}}$ becomes zero at $m_{0}=0$. Thus we have to investigate the case separately when some of parameters take zero or $\infty$. This is because we do not know what choice of the artificial parameters is preferred.

Those cases are significant in that $m_{0}=0$ or $\infty$ occasionally corresponds to the point where symmetry of the original physical model enhances; therefore the improved series may have singular structure.

\section{Multiple plateaux}

In the multi-parameter case, it is likely that there exists more than one plateau in the parameter space. If each of them can be interpreted as a physical state, some correspond to stable vacua, while others to the unstable ones. This situation is realized in Ising model [22]. When we carry out the improved perturbation for the IIB matrix model, we expect that multiple plateaux are realized corresponding respectively to different vacua with different symmetry. For this reason we must pick up all candidates of plateau and examine these carefully. 


\section{Short summary}

In this section, we discussed the concept of "plateau" and the prescription to identify them in the improved series. This improved perturbation method must be applied independently for individual observables.

To briefly summarize the procedure for each observable, we first find extrema of the series as a function of artificial parameters $m_{0}$, and then identify the accumulations of the extrema which should be considered as candidates of plateau. Next we evaluate weighted distributions in the region which encloses each of the candidates, and distinguish the plateau among them. The histograms also provide estimates of the exact value from the improved series.

This procedure assumes typical profile of the improved function. It will not be applicable when the function stays stable without forming extrema. In these cases, we have to locate the flat region referring to the information of other physical quantities, such as symmetry considerations. We also have to take care of the special hypersurface where some of parameters take zero or infinity; such cases may be relevant in the original physical models.

\section{IIB MATRIX MODEL}

In this section we apply the improved perturbation method to the IIB matrix model and investigate its non-perturbative solutions. In particular we concentrate on the free energy and the second moment of eigenvalue distribution. By comparing the values of free energy of the solutions, we can see which solution is most preferred. In the IIB matrix model, the distribution of eigenvalues is interpreted as the space-time itself. Therefore we can recognize the shape of the universe by computing the eigenvalue distribution. To be more precise, the square root of the second moment

of eigenvalues distribution gives the scale of extent to each direction of universe. The deviation of the ratio of space-time extent between directions from unity indicates the degree of anisotropy of the universe.

\section{A. Model setup}

The action of the IIB matrix model is

$$
S_{\text {OD-YM }}=\operatorname{Tr}\left(-\frac{g_{\mathrm{YM}}^{2}}{4}\left[X_{a}, X_{b}\right]^{2}-\frac{g_{\mathrm{YM}}}{2} \bar{\Psi}\left[X_{a}, \Gamma^{a} \Psi\right]\right),
$$


where $X_{a}(a=1 \ldots 10)$ and $\Psi^{\alpha}(\alpha=1 \ldots 16)$ are all $N \times N$ hermitian matrices which belong to a vector and a Majorana-Weyl spinor representation of $\mathrm{SO}(10)$, respectively. This action can be obtained by the dimensional reduction from ten-dimensional $\mathrm{U}(N)$ supersymmetric Yang-Mills action to zero dimension. It has the symmetry of all global $\mathrm{SO}(10), \mathrm{U}(N)$ and type IIB supersymmetry.

We perform the following change of scales:

$$
\begin{aligned}
X_{a} & \longrightarrow N^{\frac{1}{4}} X_{a}, \\
\Psi & \longrightarrow N^{\frac{1}{8}} \Psi, \\
g_{Y M}^{2} N & \longrightarrow \lambda .
\end{aligned}
$$

Then the action takes the form:

$$
S=N \operatorname{Tr}\left(-\frac{1}{4} \lambda\left[X_{a}, X_{b}\right]^{2}-\frac{1}{2} \sqrt{\lambda} \bar{\Psi}\left[X_{a}, \Gamma^{a} \Psi\right]\right) .
$$

In order to carry out the perturbative expansion, we add the following propagator terms to the action

$$
S_{0}=N \operatorname{Tr}\left(\frac{1}{2} \sum_{a, b=1}^{10} m_{a b} X_{a} X_{b}+\frac{1}{2} \sum_{a, b, c=1}^{10} m_{a b c} \bar{\Psi} \Gamma^{a b c} \Psi\right) .
$$

They are the most general quadratic terms. The fermionic bi-spinors are expanded by antisymmetric tensors by using gamma matrices. In the case of ten-dimensional Majorana-Weyl spinor, by considering a chirality, only gamma matrices of rank three are allowed.

The scaling behavior of the Feynman rules for the amplitude with respect to $N$ and $\lambda$ is given as follows.

$$
\begin{array}{ll}
\text { propagator for bosonic fields } X_{a}: & \sim \frac{1}{N}, \\
\text { propagator for fermionic fields } \Psi: & \sim \frac{1}{N}, \\
\text { 3-point vertex: } & \sim N \sqrt{\lambda}, \\
\text { 4-point vertex: } & \sim N \lambda .
\end{array}
$$

If we denote the genus of dual surface by $\mathfrak{g}$ and the number of faces of dual surface by $N_{F}$, we find that the contribution of a single diagram is

$$
\text { amplitude } \sim N^{2(1-\mathfrak{g})} \lambda^{\frac{N_{F}}{2}} .
$$

If we take the limit $N \rightarrow \infty$ with $\lambda$ fixed, the planar diagrams $(\mathfrak{g}=0)$ give the leading contributions. In this case the amplitude is given by

$$
\text { amplitude } \sim N^{2} \sum_{n} \lambda^{n} f^{n}
$$


Free energy $F$ is evaluated by the sum of connected bubble diagrams in terms of propagators $m_{a}$ and $m_{a b c}$ as

$$
\frac{F}{N^{2}}=-\ln \left(\frac{1}{m}\right)+\cdots .
$$

The expectation value of the second momentum of eigenvalue distributions is obtained by the derivatives of free energy with respect to the parameters.

$$
\begin{aligned}
\frac{1}{N^{2}} \frac{\partial F}{\partial m_{a}} & =\left\langle\frac{1}{N} \operatorname{Tr} X_{a}^{2}\right\rangle \\
& =\frac{\partial}{\partial m_{a}}\left(-\ln \left(\frac{1}{m}\right)+\cdots\right) .
\end{aligned}
$$

The original IIB matrix model does not have the propagator term eq. (32). Therefore we evaluate the observables at $m_{a}=m_{a b c}=0$. They are singular in this region of parameters in the above perturbation. We use the improved perturbation to extrapolate to the massless case.

In general, all parameters $m_{a b}, m_{a b c}$ have to be taken into account. We may perform SO(10) rotation to reduce $m_{a b}$ to the diagonal form at the stage of action. We will impose further restrictions from the practical reason in the later sections.

Before going into a concrete computation, we make some assumptions. In computing the free energy we have to evaluate all connected bubble diagrams. As we proceed to the higher order of perturbation, the number of diagrams becomes enormous. We consider such a situation that the $\mathrm{U}(N)$ symmetry stays intact. Throughout this article we concentrate on the spontaneous breakdown of Lorentz symmetry ${ }^{3}$. Then we can omit the tadpole amplitude

$$
\left\langle\left(X^{a}\right)_{i j}\right\rangle=0
$$

for the matrix corresponding to the adjoint representation of $\mathrm{SU}(N)$ subalgebra. By the argument of the gauge symmetry alone we can not forbid the tadpole amplitude of $U(1)$ part. If it takes non-zero expectation value $x^{a}$, we perform the field redefinition

$$
X^{a} \rightarrow X^{a}+\frac{x^{a}}{N} \mathbf{1}_{N \times N}
$$

By this redefinition we can set the tadpole amplitude to zero,

$$
\left\langle\operatorname{Tr}\left(X^{a}\right)\right\rangle=0 .
$$

\footnotetext{
${ }^{3}$ The breakdown of $\mathrm{U}(N)$ gauge symmetry will be discussed in the subsequent paper [35].
} 
As we show later the effective action bears dynamical mass term. Then the zero point of free energy is shifted due to the redefinition of fields by eq. (38). Thus we can not use the zero of free energy as a characteristics of supersymmetry.

Under the assumption above, the sum of connected bubble diagrams reduces to the sum of oneparticle irreducible (1PI) bubble diagrams. Thus far we have to evaluate 1PI bubble diagrams in order to obtain free energy.

In the estimation of 1PI bubble diagrams we can drastically simplify the computation by using 2PI free energy [27]. At first we compute 2 PI graph amplitude $G_{2 \mathrm{PI}}$ by using full propagators

$$
\begin{aligned}
\left\langle\left(X_{a}\right)_{i j}\left(X_{b}\right)_{k l}\right\rangle & =\frac{1}{N} c_{a b} \delta_{i l} \delta_{j k}, \\
\left\langle\left(\Psi_{\alpha}\right)_{i j}\left(\Psi_{\beta}\right)_{k l}\right\rangle & =\frac{1}{N} \frac{1}{3 !} u_{a b c}\left(C \Gamma^{a b c}\right)_{\alpha \beta} \delta_{i l} \delta_{j k},
\end{aligned}
$$

in place of perturbative propagators $m_{a}, m_{a b c}$. Here we use double line notation, and $C$ is the charge conjugation matrix.

From $G_{2 \mathrm{PI}}$ we obtain 1 PI bubble amplitude $F_{1 \mathrm{PI}}$ by Legendre transformation $[36]^{4}$.

$$
\begin{gathered}
\mathfrak{F}\left(m_{a}, m_{a b c} ; c_{a}, u_{a b c}\right) \equiv G_{2 \mathrm{PI}}\left(m_{a}, m_{a b c}\right)+\frac{1}{2} \sum_{a=1}^{10} m_{a} c_{a}-\frac{1}{2} \sum_{a, b, c=1}^{10} m_{a b c} u_{a b c}, \\
0=\left.\frac{\partial \mathfrak{F}}{\partial m_{a}}\right|_{c_{a}=\bar{c}(m)}, \\
F_{1 \mathrm{PI}}=\left.\mathfrak{F}\right|_{c_{a}=\bar{c}(m)} .
\end{gathered}
$$

Eq. (42) corresponds to the Schwinger-Dyson equation which relates full propagators $c_{a}$ and $u_{a b c}$ to perturbative propagators $m_{a}$ and $m_{a b c}$, and eq. (43) produce the 1PI amplitude using bare propagators $m_{a}, m_{a b c}$.

In the present article, we use the result of the perturbative expansion up to eighth order in $\lambda$ of Ref. [29].

\section{B. Ansatz}

In this subsection, we make assumptions for the pattern of symmetry breakdown. In the case of the IIB matrix model, the total number of artificial parameters is quite large, namely, 10 real numbers for $m_{a b}$ (assumed to be diagonalized by $\mathrm{SO}(10)$ rotation), and 120 for $m_{a b c}$. It will demand

\footnotetext{
${ }^{4}$ For details see also Ref.[27.
} 
an enormous effort to search for plateau in this vast space of parameters. Therefore, we impose restrictions on the configuration by considering symmetry to diminish the number. In concrete manner, we make same assumptions for the full propagators $c_{a b}$ and $u_{a b c}$ because we compute 2PI bubble diagrams by using these parameters.

In the former works[26, 27, 28, 29], the configurations called $\mathrm{SO}(d)$ ansatz have been intensively examined which preserve $\mathrm{SO}(d)$ rotational symmetry. The guideline of choice is described as follows. First, $\mathrm{SO}(d)$ subgroup of $\mathrm{SO}(10)$ is chosen to which directions the expectation values of fermionic two-point function $u_{a b c}$ are zero. $d$ is taken from 1 to 9. Toward the rest of the directions $u_{a b c}$ may have non-zero value. Since $u_{a b c}$ is a rank three anti-symmetric tensor, a single non-zero component of $u_{a b c}$ brings out three-dimensional subspace by permutation of indices. Thus, $(10-d)$ dimensional part would naturally be decomposed into multiples of three-dimensional blocks. Furthermore, those blocks are subjected to the permutation symmetry under the interchange with each other. In this way, $\mathrm{SO}(d)(d=1 \cdots 7)$ symmetric ansatz has been investigated in former works.

Among those choices shown above, we examine $d=4$ and $d=7$ cases in particular. It is reported in Ref. [27] that $d=5$ and 6 cases reduce to $\mathrm{SO}(7)$ ansatz, while $d=2$ and 3 cases to $\mathrm{SO}(4)$ ansatz. $d=1$ had no solution.

The preserved symmetry and the explicit forms of the full propagators for $d=4$ and $d=7$ cases are given as follows.

SO(7) ansatz: $\mathrm{SO}(7) \times \mathrm{SO}(3)$

$$
c_{a b}=\operatorname{diag}\left(7 c_{1} \text { 's, } 3 c_{2} \text { 's }\right), \quad \not u=u \Gamma^{8,9,10},
$$

SO(4) ansatz: $\mathrm{SO}(4) \times \mathrm{SO}(3) \times \mathrm{SO}(3) \times Z_{2}$

$$
c_{a b}=\operatorname{diag}\left(4 c_{1} \text { 's, } 6 c_{2} \text { 's }\right), \quad \not=\frac{u}{\sqrt{2}}\left(\Gamma^{5,6,7}+\Gamma^{8,9,10}\right),
$$

the $Z_{2}$ factor stands for the permutation symmetry between two $\mathrm{SO}(3)$ factors.

For the ansatz presented above, we evaluate the free energy given by the sum of 1PI bubble diagrams. It is obtained as a series of the coupling constant $\lambda$ whose coefficients are functions of the parameters $m_{1}, m_{2}$, and $m$. Next, we calculate the second moment of eigenvalue distribution by differentiating the free energy with respect to $m_{1}$ and $m_{2}$. Finally, we apply the improved perturbation method to these perturbative series:

$$
\begin{aligned}
F^{N}\left(\lambda, m_{1}, m_{2}, u\right) & \longrightarrow \widetilde{F}^{N}\left(\lambda,\left(m_{0}\right)_{1},\left(m_{0}\right)_{2}, u_{0}\right) \\
& =\left.F^{N}\left(g \lambda,\left\{m_{0}\right\}+g\left(\{m\}-\left\{m_{0}\right\}\right)\right)\right|_{g^{N}, g \rightarrow 1,\{m\}=0}
\end{aligned}
$$


$\{m\}$ and $\left\{m_{0}\right\}$ represent collectively the set of parameters $\left\{m_{1}, m_{2}, m\right\}$ and $\left\{\left(m_{0}\right)_{1},\left(m_{0}\right)_{2}, m_{0}\right\}$, respectively. We put $m_{1}=m_{2}=m=0$ so that it describes the original IIB matrix model. At this stage, we set $\lambda=1$. It is because the coupling constant $\lambda$ can be absorbed by the redefinition of the fields.

We introduce the new variables $x_{i}$ as

$$
x_{1} \equiv \frac{1}{\left(m_{0}\right)_{1}}, \quad x_{2} \equiv \frac{1}{\left(m_{0}\right)_{2}}, \quad x_{3} \equiv \frac{1}{m_{0}},
$$

and use these parameters in the following section.

\section{Results in $\mathrm{SO}(4)$ ansatz}

In this subsection, we present the results in the case of $\mathrm{SO}(4)$ ansatz. We apply the improved perturbation method to the perturbative series for the free energy, the second moment of eigenvalue distribution for $X^{a}(a=1 \cdots 4)$, which is denoted by $c_{1}$, and that for $X^{a}(a=5 \cdots 10)$, which is denoted by $c_{2}$.

As explained in Sec. II we first search for the extrema of these functions with respect to the artificial parameters $x_{i}$. Then, we find the accumulations of extrema and consider them as the candidates of plateau. We compute the weighted distribution of the functions in those regions. We use here the first derivatives (25) and the second derivatives (26) in particular. Finally, we identify the region as plateau in which two distributions overlap with each other. The graphs of distributions in individual regions are shown in Appendix $\mathrm{A}$.

\section{Free energy}

Fig. 8 shows the distribution of extrema of the improved series of free energy. As is seen in Fig. 8, there are several accumulations of extrema. We pick up some accumulations as the candidates of plateaux and compute the distributions with weight functions $w_{1}$ and $w_{2}$. Besides most of accumulations show the hopeless distributions which spread randomly, two regions $\mathrm{A}$ and B which are depicted in Fig. 8 have a hopeful distribution. In the following, we refer to only hopeful candidates.

Two regions A and B are hopeful candidates of plateau, although the behavior of the function of the seventh order in region $\mathrm{A}$ is rather unstable. The similar situation also occurred in the previous work [29] in which the positions of extrema show a different pattern for the seventh order 


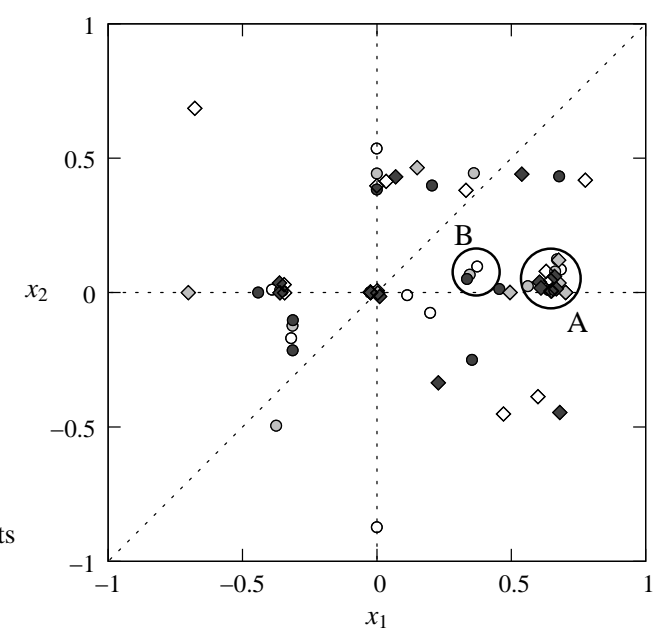

$\begin{array}{ll}\text { order 3 } & \circ \\ \text { order 4 } & \diamond \\ \text { order 5 } & \circ \\ \text { order 6 } & \diamond \\ \text { order 7 } & \vdots \\ \text { order 8 } & \diamond\end{array}$

FIG. 8: Distribution of extrema of improved free energy for $\mathrm{SO}(4)$ ansatz plotted in $x_{1}-x_{2}$ plane.

of perturbation. In the region A we read the value of free energy to be $-1 \sim 2$. And in the region $\mathrm{B}$, we also read the value of free energy to be $-1 \sim 2$.

From this accordance between region $\mathrm{A}$ and $\mathrm{B}$ about the value of free energy, we may expect that two regions belong to one plateau. However from the examination of the other observables in the later subsections, we conclude that two regions are distinguished each others and there are two plateaux.

2. $c_{1}$

Fig. 9 shows the distribution of extrema of the improved series of $c_{1}$. As mentioned in former section, although $c_{1}$ is the derivative function of free energy with respect to $m_{1}$, we have to apply the improved perturbation separately. It is because the behavior of the function becomes singular in the region of moduli space that corresponds to the massless case. It is seen by comparing Fig. 8 with Fig. 9. Two distributions of extrema show the different pattern.

Among accumulations, the regions $\mathrm{A}, \mathrm{B}$ and $\mathrm{C}$ are promised candidates of plateau. The distribution in the region A shows a strange behavior. As the order of perturbation increases, the value at the peak of distribution shifts to the right. When we approximate an infinitely large value by a finite series, such a behavior may often be observed. Therefore this property indicates that the exact value is quite large. Up to the eighth order of perturbation, we conclude that $c_{1}$ takes value of $40 \sim 60$. From the distribution in the region $\mathrm{B}$, we read the value of $c_{1}$ to be $1.5 \sim 2.1$. Although regions $\mathrm{A}$ and $\mathrm{B}$ point the similar value of free energy, these regions shows clearly different distri- 


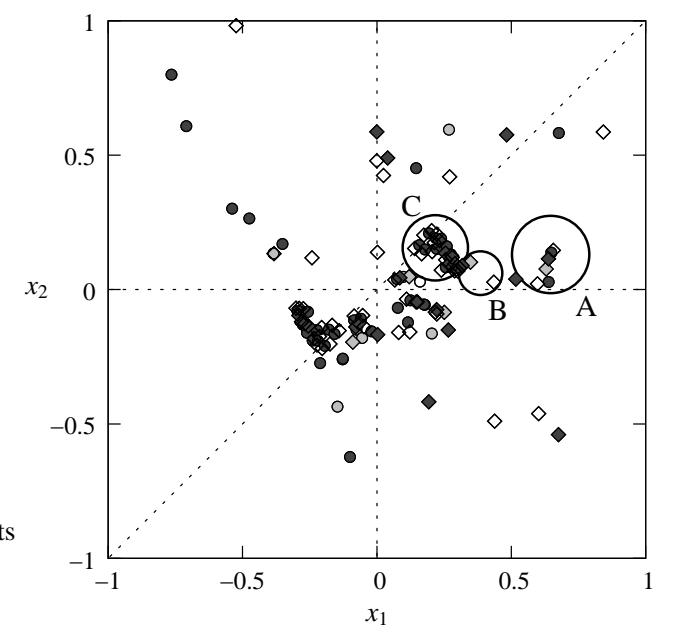

$\begin{array}{ll}\text { order 3 } & \circ \\ \text { order 4 } & \diamond \\ \text { order 5 } & \circ \\ \text { order 6 } & \diamond \\ \text { order 7 } & \bullet \\ \text { order 8 } & \diamond\end{array}$

FIG. 9: Distribution of extrema of the improved $c_{1}$ for $\mathrm{SO}(4)$ ansatz.

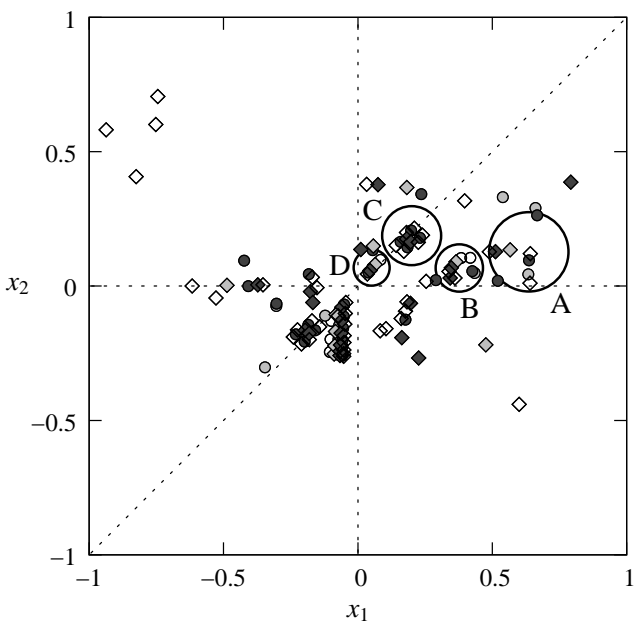

$\begin{array}{ll}\text { order 3 } & \circ \\ \text { order 4 } & \diamond \\ \text { order 5 } & \circ \\ \text { order 6 } & \diamond \\ \text { order 7 } & \diamond \\ \text { order 8 } & \diamond\end{array}$

FIG. 10: Distribution of extrema of the improved $c_{2}$ for $\mathrm{SO}(4)$ ansatz.

butions with respect to $c_{1}$. Therefore we conclude that these regions belong to different plateaux. On the other hand, from the region $\mathrm{C}$, we read the value of $c_{1}$ to be $0.25 \sim 0.26$. Thus we conclude that there are three plateaux. In particular, it is interesting that the region $\mathrm{C}$ shows the same value as the region $\mathrm{C}$ of $c_{2}$ and $\mathrm{SO}(7)$ ansatz which will be shown in later subsections. The significance of this accordance will be discussed later in Subsection IVE

3. $c_{2}$

Fig. 10 shows the distribution of extrema of the improved series of $c_{2}$. We consider the regions A, B, C and D as candidates of plateau. From the distribution in the region A, we read the value of 


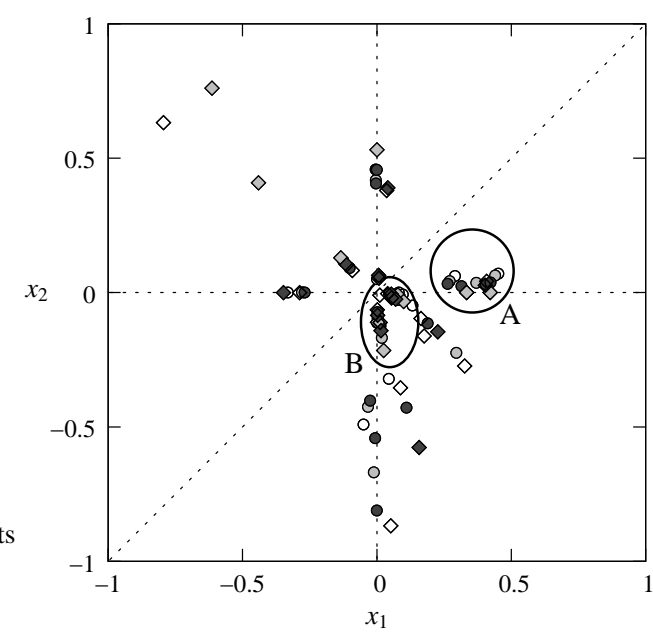

order 3

order 4

order 6

order 8

FIG. 11: Distribution of extrema of the improved free energy for $\mathrm{SO}(7)$ ansatz plotted in $x_{1}-x_{2}$ plane.

$c_{2}$ to be $0.0 \sim 0.2$. From the distribution in the region $\mathrm{B}$, we read the value of $c_{2}$ to be $0.13 \sim 0.15$. From the distribution in the region $\mathrm{C}$, we read the value of $c_{2}$ to be $0.25 \sim 0.26$.

In the similar fashion, region $\mathrm{D}$ indicates that $c_{2}$ takes the value $0.21 \sim 0.25$. From the similarity of the distributions in the region $\mathrm{C}$ and $\mathrm{D}$, we conclude that these two regions belong to one plateau. Thus we choose the region $\mathrm{C}$ as a representative of this plateau.

\section{Results in $\mathrm{SO}(7)$ ansatz}

In this subsection, we show the results for the case of $\mathrm{SO}(7)$ ansatz. The method of analysis is the same as that in the $\mathrm{SO}(4)$ case. The graphs of distributions in individual regions are shown in Appendix $\mathrm{A}$

\section{Free energy}

Fig. 11 shows the distribution of extrema of the improved series of free energy. Though it seems that the region B contains three distinct accumulations, we treat them as one region. It is because the distributions of three regions show the similar shape, then it is indicated that all three accumulations belong to one structure. Thus, there are two candidates of plateau.

From the distribution in the region $\mathrm{A}$, we read the value to be $5 \sim 7$. From the distribution in the region $\mathrm{B}$, we read the value of the free energy to be $-10 \sim 0$. The extrema in the region $\mathrm{B}$ are considered to be overshoots, because the weighted distribution with the second derivative 


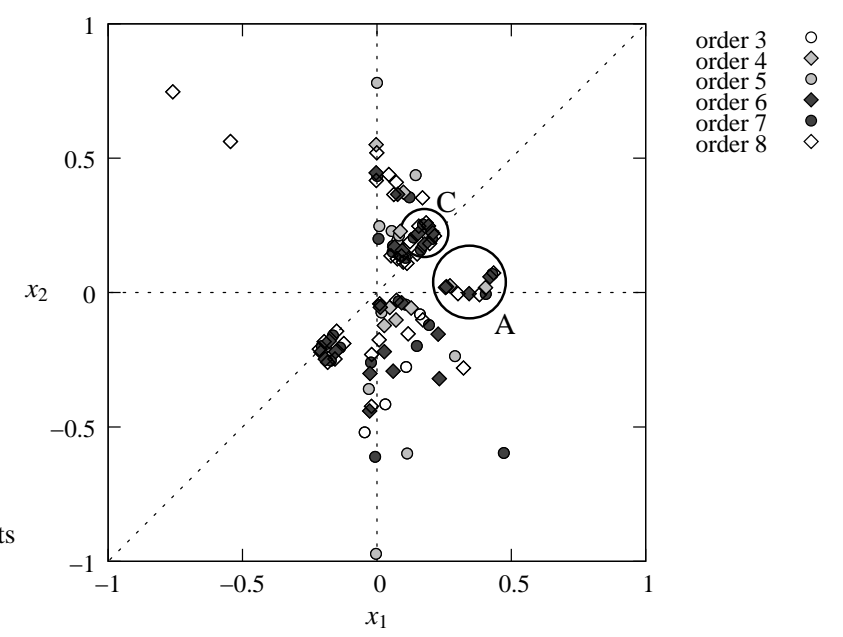

FIG. 12: Distribution of extrema of improved $c_{1}$ for $\mathrm{SO}(7)$ ansatz.

deviates from that with the first derivative, and it is a characteristic feature of overshoot. Moreover, the behavior of the improved series of other observables around this region looks despairing. We conclude that it is indeed the overshoot.

2. $c_{1}$

Fig. 12 shows the distribution of extrema of the improved series of $c_{1}$. Regions A and C are hopeful accumulations. From the distribution in the region $\mathrm{A}$, we read the value of $c_{1}$ to be $0.7 \sim 0.8$. From that in the region $\mathrm{C}$, we read the value of $c_{1}$ to be $0.25 \sim 0.26$.

3. $c_{2}$

Fig. 13 shows the distribution of extrema of the improved series of $c_{2}$. Regions A and C are hopeful regions. From the distribution in the region $\mathrm{A}$, we read the value of $c_{2}$ to be $0.08 \sim 0.12$. From that in the region $\mathrm{C}$, we take the value of $c_{2}$ to be $0.25 \sim 0.26$.

\section{E. $\mathrm{SO}(10)$-symmetric vacuum}

From the investigation of the observables $c_{1}$ and $c_{2}$, we find regions in both $\mathrm{SO}(4)$ and $\mathrm{SO}(7)$ ansatz that have an interesting property. It is shown by region $\mathrm{C}$ in Figs. 9 and 10 for $\mathrm{SO}(4)$ ansatz, and in Figs. 12 and 13 for $\mathrm{SO}(7)$ ansatz as well. The approximate values of $c_{1}$ and $c_{2}$ estimated 


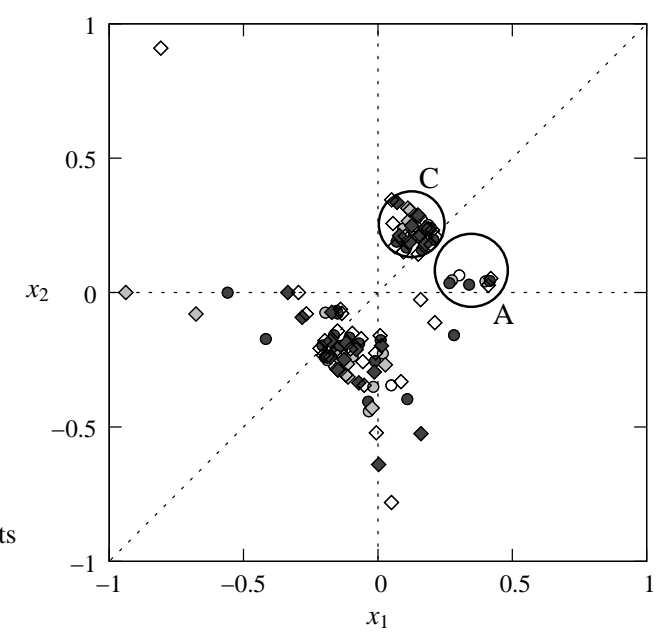

order $3 \quad 0$

order 4

order 6

order 7

order 8

FIG. 13: Distribution of extrema of improved $c_{2}$ for $\mathrm{SO}(7)$ ansatz.

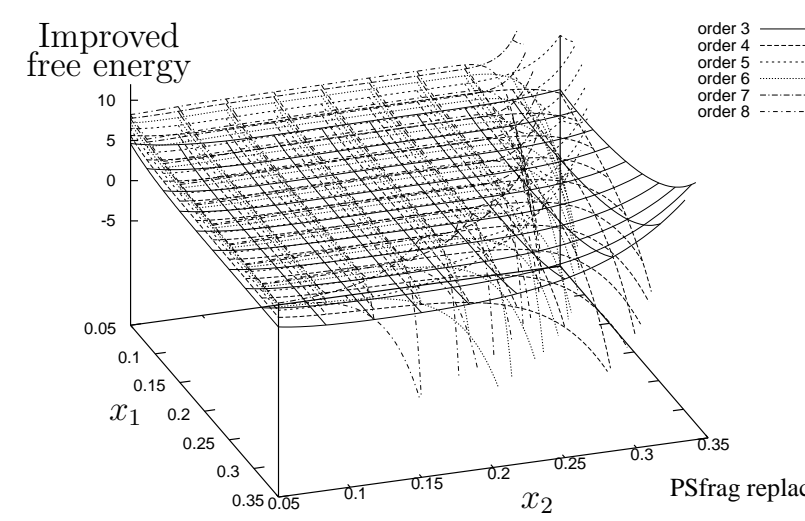

(a)

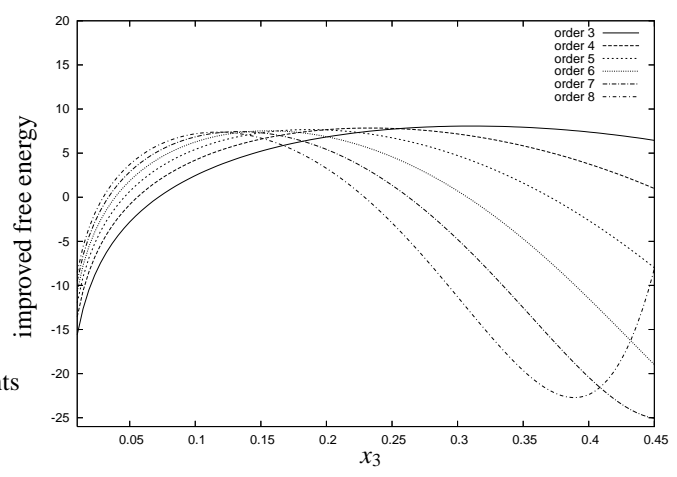

(b)

FIG. 14: Dependence of the improved free energy with respect to $x_{1}$ and $x_{2}$ at $x_{3}=0.1$ (a), and $x_{3^{-}}$ dependence of the improved free energy at $x_{1}=x_{2}=0.15$ (b) for $\mathrm{SO}(4)$ ansatz.

from the distributions are $0.25 \sim 0.26$, and they are almost the same. In addition, the region $\mathrm{C}$ is located at $x_{3} \sim 0$, and thus it implies that the bi-fermionic variable $u_{1}$ vanishes. Therefore, we identify this region as the plateau that corresponds to the $\mathrm{SO}(10)$-symmetric vacuum.

It may seem strange that there is no such accumulation of extrema found for the improved free energy around region $\mathrm{C}$ above. In fact, this region appears as "smooth plateau" discussed in Sec. III] By plotting the dependence of the improved free energy (Fig. 14), we can recognize that the improved free energy stays almost constant in region $\mathrm{C}$, and thus the minimal sensitivity is indeed realized there. The approximate value of the free energy is obtained from the distribution 
shown in Appendix $\mathrm{A}$ as $6 \sim 7$.

The above observations are obtained for both $\mathrm{SO}(4)$ ansatz and $\mathrm{SO}(7)$ ansatz, and the approximate values of the free energy, $c_{1}$, and $c_{2}$ are the same between those two ansatz. It is consistent with the speculation that these solutions obtained for two distinct ansatz are actually identical, in which the $\mathrm{SO}(10)$-symmetry is realized.

\section{F. Results in IIB matrix model}

In the examinations of three observables, free energy, $c_{1}$ and $c_{2}$, for the $\mathrm{SO}(4)$ ansatz we find three sets of candidate of plateau.

First plateau (we call this region as region A) indicates that the value of free energy is $-1 \sim 2$, that of $c_{1}$ reaches 50 and that of $c_{2}$ is less than 0.2 up to the eighth order of perturbation. This plateau corresponds to an anisotropic vacuum that preserves $\mathrm{SO}(4)$ subgroup. The ratio of extent of the eigenvalue distributions between the 4-dimensional part and the 6-dimensional part attains at 20 at the stage of the eighth order of perturbation. The behavior of distributions as increase of a order of perturbation indicates that the exact value of ratio is quite large. This solution has a property that the degree of anisotropy is large (probably infinite). Next plateau (we call this region as region $\mathrm{B}$ ) indicates that the value of free energy is $-1 \sim 2$ and that of $c_{1}$ is $1.5 \sim 2.1$ and that of $c_{2}$ is $0.13 \sim 0.15$. This plateau also corresponds to an anisotropic vacuum that preserves $\mathrm{SO}(4)$ subgroup. The ratio of extent of the eigenvalue distributions between the 4-dimensional part and the 6-dimensional part is obtained by $3.1 \sim 4.0$. This solution has a property that the degree of anisotropy is finite.

The third candidate appears in a different manner. This is the plateau corresponding to a $\mathrm{SO}(10)$-symmetric vacuum which was discussed in the previous subsection. For the observables $c_{1}$ and $c_{2}$, it indicates that both of $c_{1}$ and $c_{2}$ take the value $0.25 \sim 0.26$. For the free energy this plateau appears as "smooth plateau". By computing the distribution of free energy we conclude that the approximate value of free energy is $6 \sim 7$. The graphs of distributions in this regions are shown in Appendix A

Similar argument can be applied to the case of $\mathrm{SO}(7)$ ansatz. There are two plateaux; one of which indicates that the value of free energy is $5 \sim 7$ and that of $c_{1}$ is $0.7 \sim 0.8$ and that of $c_{2}$ is $0.08 \sim 0.12$, and it corresponds to an anisotropic vacuum that preserves $\mathrm{SO}(7)$ subgroup. The ratio of the extent between the 7-dimensional part and the remaining 3-dimensional part is $2.4 \sim 3.1$. 
The other plateau corresponds to a $\mathrm{SO}(10)$-symmetric vacuum. This plateau indicates that the value of free energy is $6 \sim 7$ and that of $c_{1}$ and $c_{2}$ is $0.25 \sim 0.26$. Those values are the same as those obtained for the third plateau of $\mathrm{SO}(4)$ ansatz. It is consistent with the speculation that they are actually identical, and $\mathrm{SO}(10)$ symmetry is realized.

\section{CONCLUSION}

We have investigated the properties of the improved perturbation method in much detail. This method is a sort of systematic optimization of a series expansion obtained by the perturbation theory, achieved by reorganizing the series with the artificially introduced parameters. In particular, we focus on the realization of the principle of minimal sensitivity, which provides a condition for the parameters that the improved series should be least dependent on them. A region in the parameter space that satisfies the above condition is called as "plateau". The exact value of the series would be reproduced on the plateau. Thus the efficient scheme for the identification of the plateau and evaluation of the approximate value becomes a major issue in the application of the improved perturbation method.

We proposed a new scheme for evaluating the improved series which yields good approximate values. It relies on the distribution profile of the series that is weighted by the first and the second derivatives with respect to the artificial parameters. By incorporating those weightings, the distribution becomes more sensitive to such a behavior that the series stays almost constant in a certain region of the parameters, i.e. the plateau condition be satisfied. It is also noted that this scheme works effectively even when there are a number of parameters.

We insist that the improved perturbation method should be applied independently for each individual observable of interest. It is because the prescription of improvement is quite nonlinear and the formation of plateau may occur in a different manner especially at low orders of perturbation. If they are supposed to coincide at high enough orders, the information of plateau of other observables may help to clarify the behavior of the improved series. Thus those information should be referred synthetically.

We studied the IIB matrix model by the improved perturbation method in order to examine the non-perturbative solutions of the model. We concentrate on the possibility of the spontaneous breakdown of Lorentz symmetry in the present article. We applied our scheme to the analysis of the improved series up to eighth order of perturbation for $\mathrm{SO}(4)$ and $\mathrm{SO}(7)$ ansatz that have 
been obtained in former works. As a result, we found a new solution that corresponds to the tendimensional universe besides the four- and seven-dimensional solutions already found previously. A key to this detection resides in that we performed the analysis independently for individual observables and combined the information on the plateau of them.

The existence of the plateau of the $\mathrm{SO}(10)$-symmetric vacuum is quite substantial because it provides an evidence for the validity of application of the improved perturbation method to the IIB matrix model. It ensures us that the investigation encloses the whole moduli space of the model including the $\mathrm{SO}(10)$ symmetry and that the improved series indeed describes the original $\mathrm{SO}(10)$ symmetric IIB matrix model. It is a non-trivial issue when the model exhibits phase transitions, as was discussed in the application to the Ising model [22].

Based on our new scheme for the improved perturbation method, we found that the value of free energy becomes large in the order of four-, seven- and ten-dimensional universe. Thus we conclude that the four-dimensional universe is more preferred than seven- and ten-dimensional universe.

It is interesting that there are two distinct solutions that correspond to four-dimensional universe. One is the solution in which the degree of anisotropy is quite large, and the other is the solution in which the degree of anisotropy is finite. The estimated values of free energy are almost the same for those two solutions. It may suggest an interesting possibility that the inflation is caused by the phase transition from one $\mathrm{SO}(4)$ vacuum with finite degree of anisotropy to the other $\mathrm{SO}(4)$ vacuum that has infinitely large extent in four directions, i.e. our universe.

\section{Acknowledgments}

The authors are grateful to H. Kawai and T. Matsuo for valuable discussions. Y. S. is supported by the Special Postdoctoral Researchers Program at RIKEN.

\section{APPENDIX A: DETAILS OF ANALYSIS IN IIB MATRIX MODEL}

In this appendix we show the graphs of distributions in individual ansatz and these regions which are candidates of plateaux.

As we can see in Fig,8, 13, for each observables there are many accumulations of extrema. We must compute the distribution with weight function $w_{1}, w_{2}$ for each region. Most of regions show 
hopeless distributions. Then we select only the hopeful regions here and depict the graphs of the distributions in these regions. The locations of these regions in the artificial parameter space are depicted in Fig, $8,13$. 


\section{1. $\mathrm{SO}(4)$ ansatz}

a. Free energy

\section{Region A}
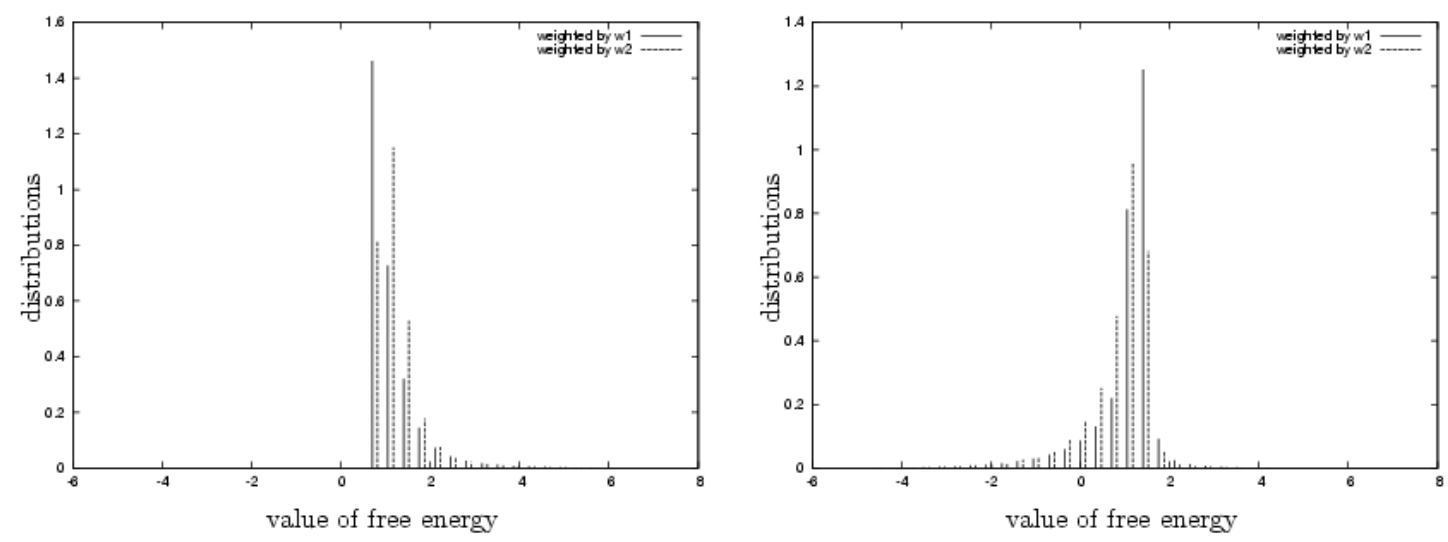

(a) order 3

(b) order 4
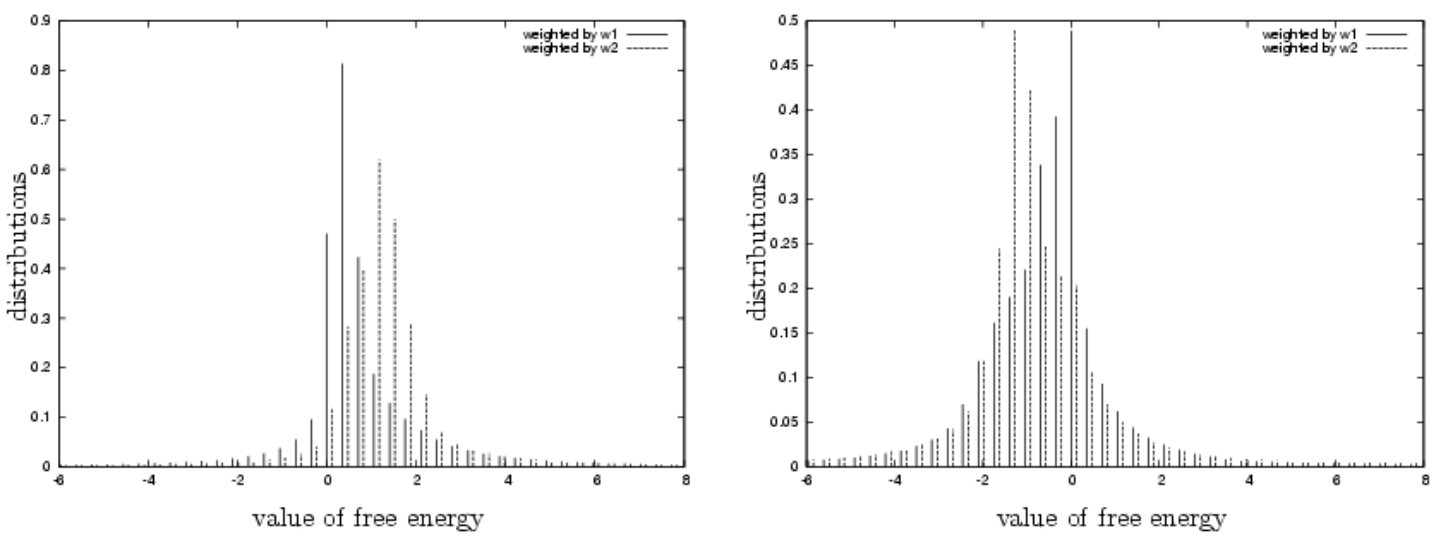

(c) order 5

(d) order 6
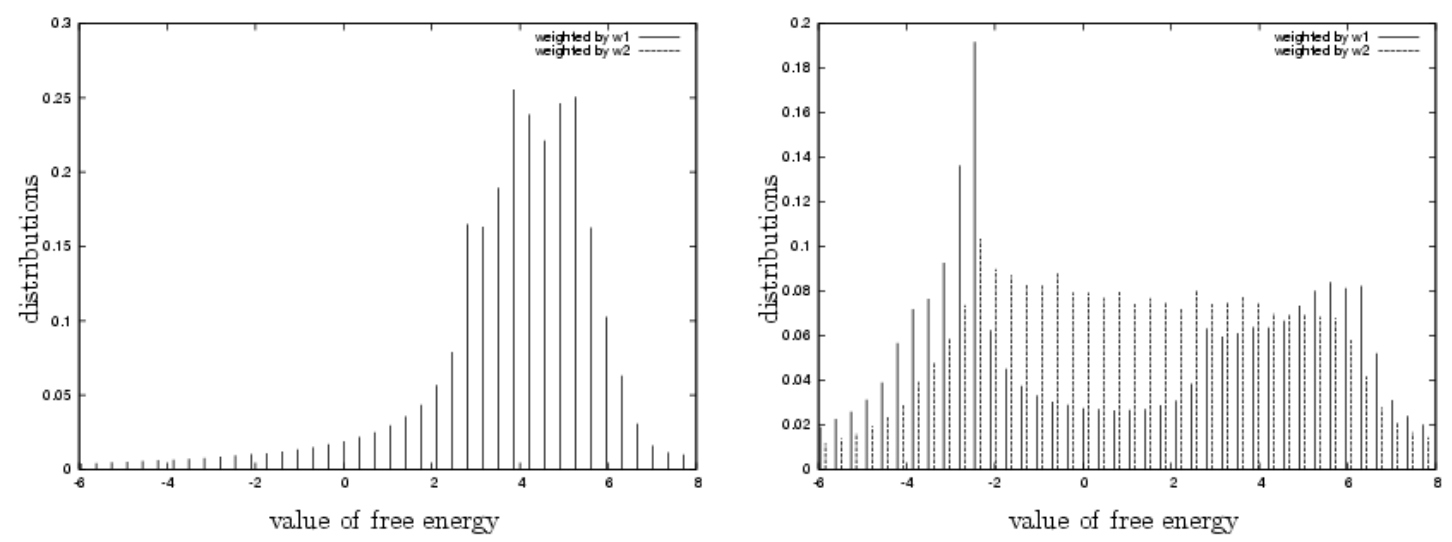

(e) order 7

(f) order 8 


\section{Region B}
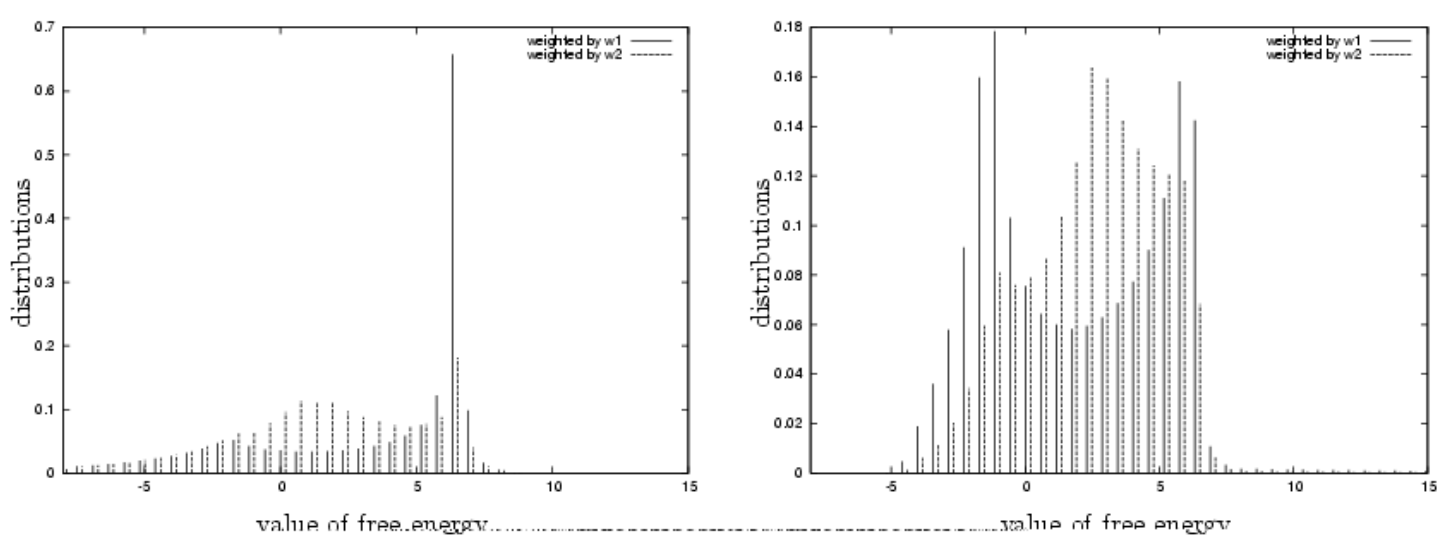

(a) order 3

(b) order 4
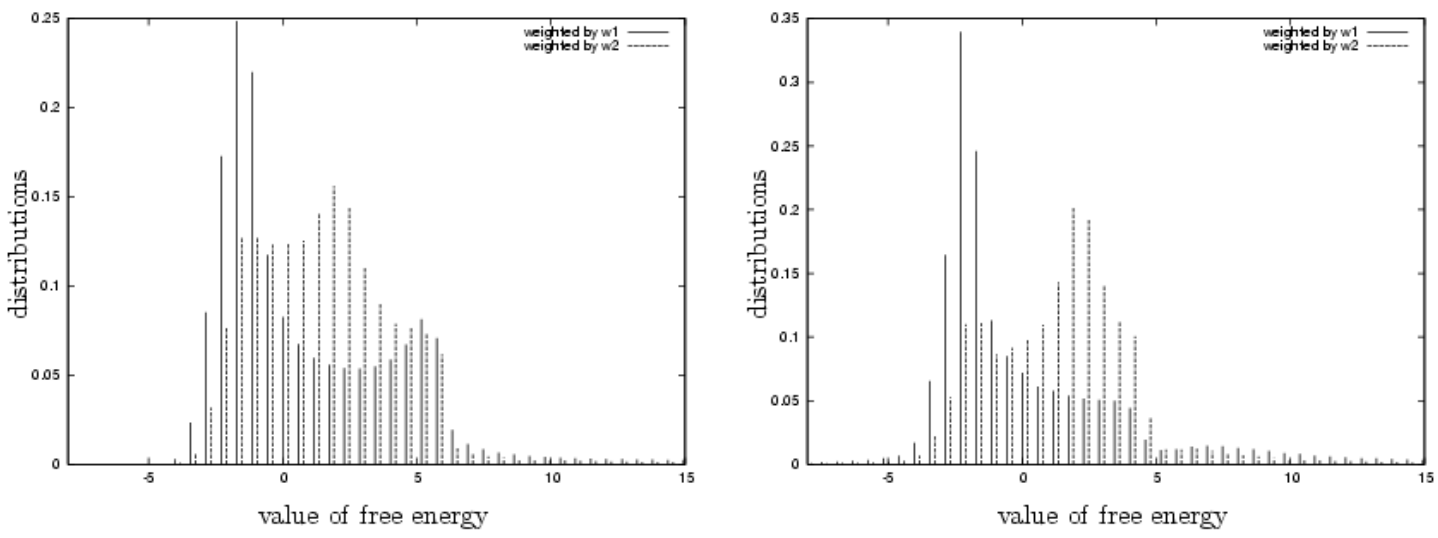

(c) order 5

(d) order 6

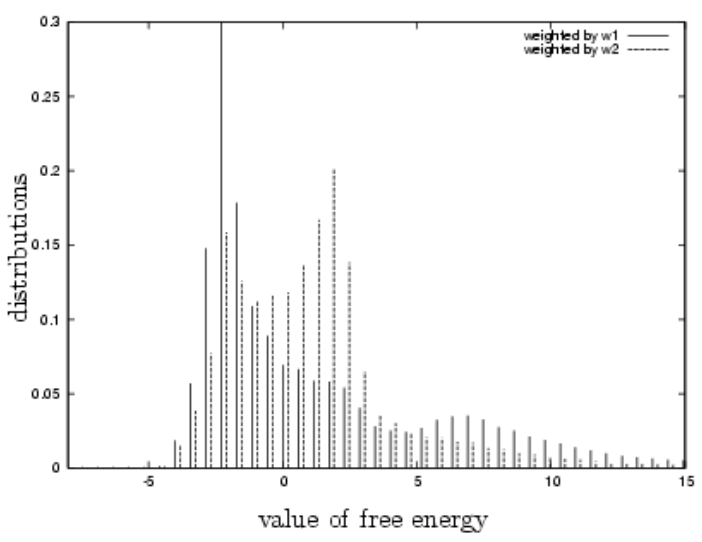

(e) order 7

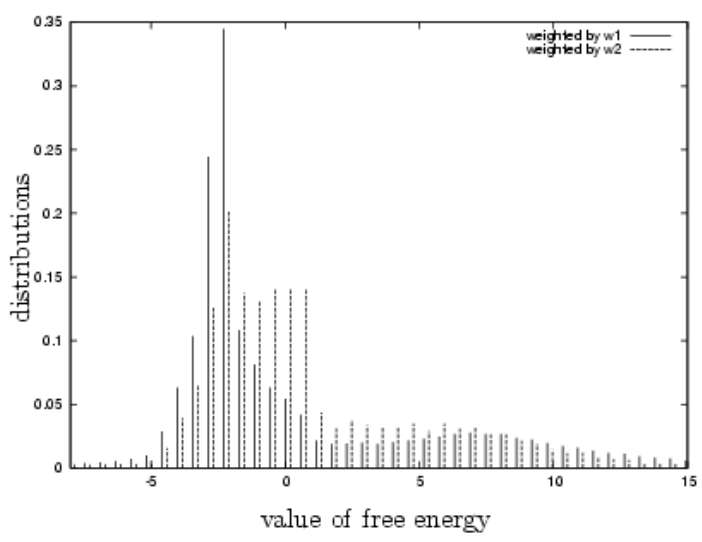

(f) order 8 
b. $c_{1}$

Region A
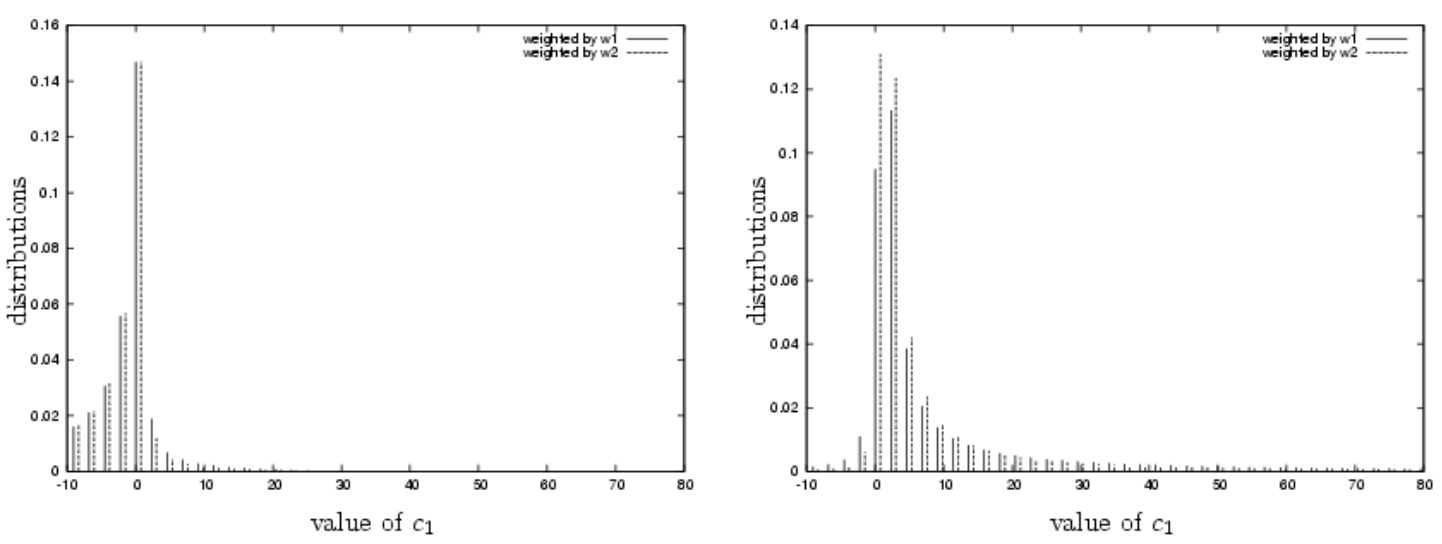

(a) order 3

(b) order 4
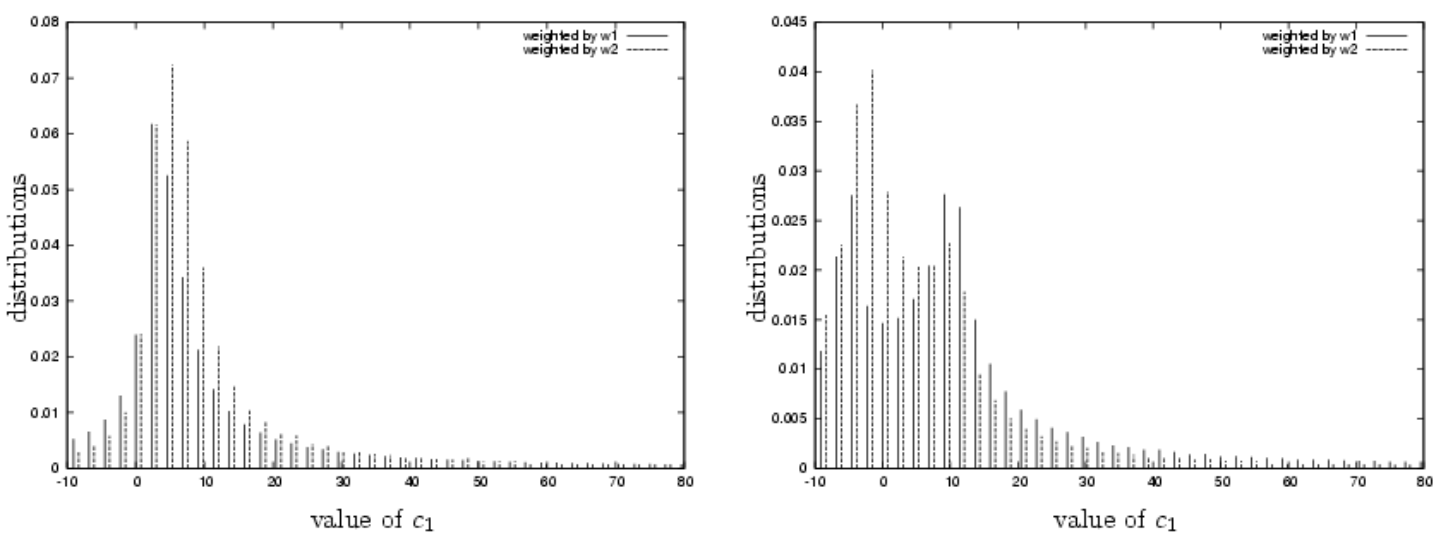

(c) order 5

(d) order 6
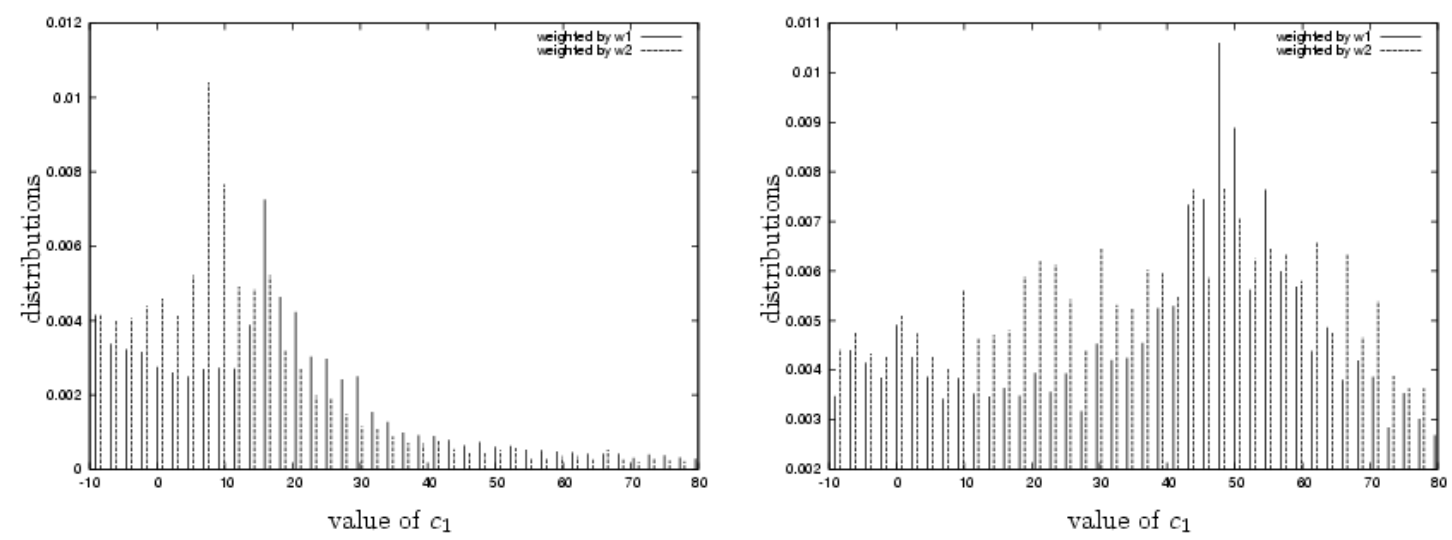

(e) order 7

(f) order 8 
Region B

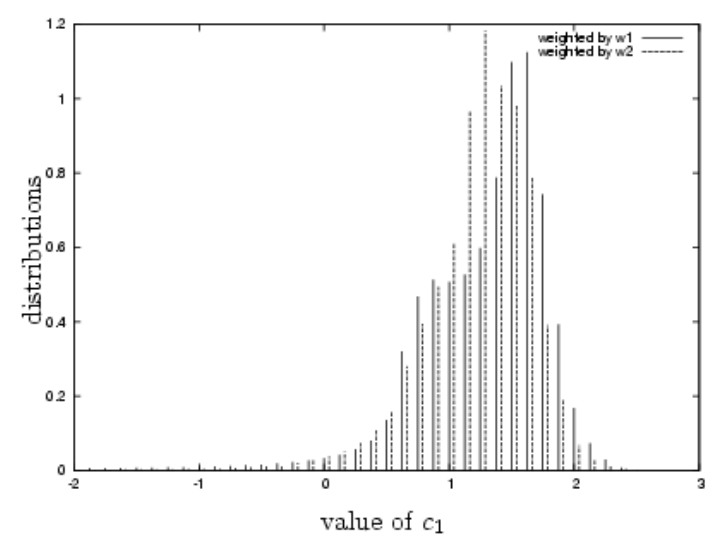

(a) order 3

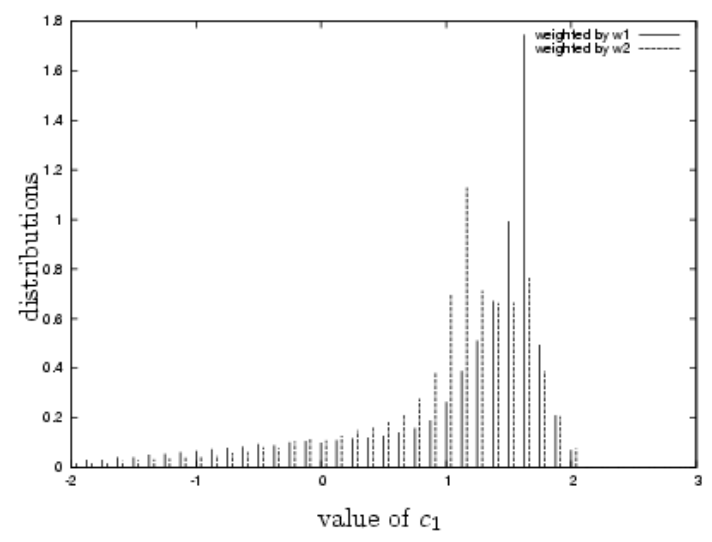

(c) order 5

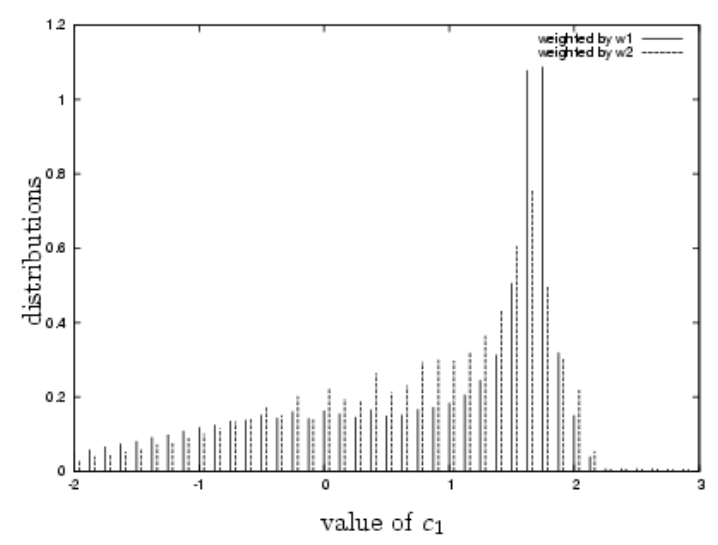

(e) order 7

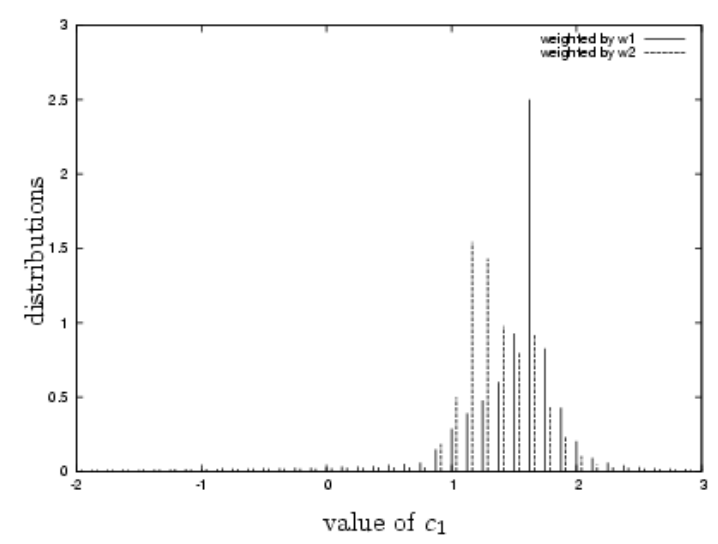

(b) order 4

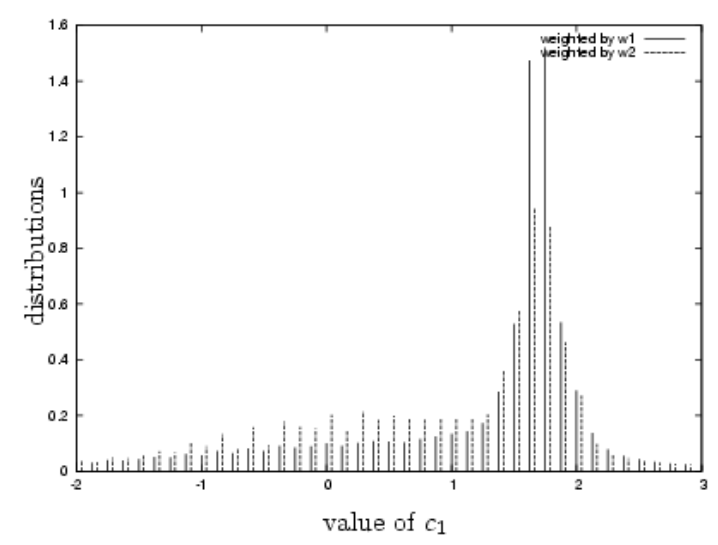

(d) order 6

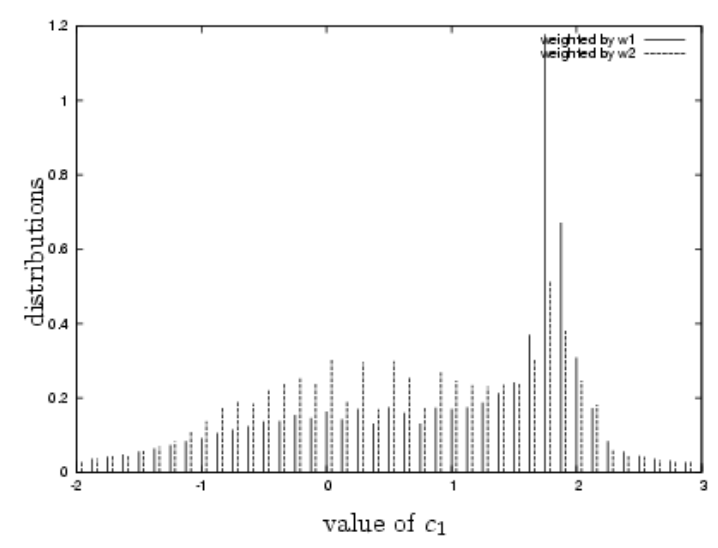

(f) order 8 
Region C
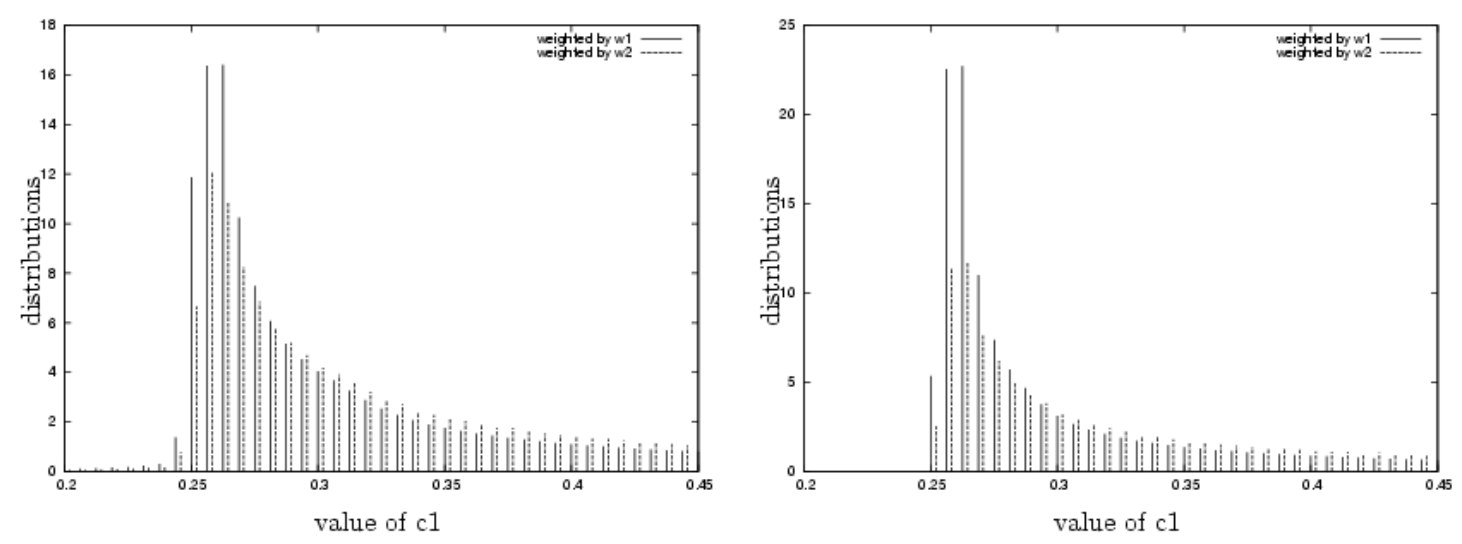

(a) order 3

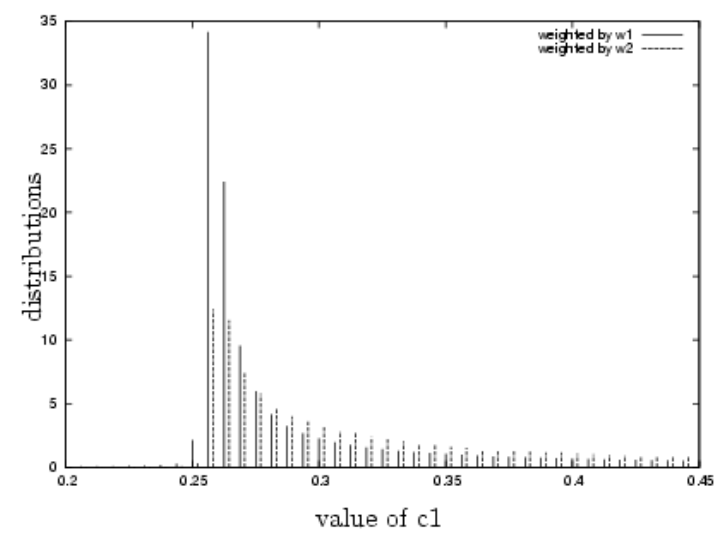

(b) order 4

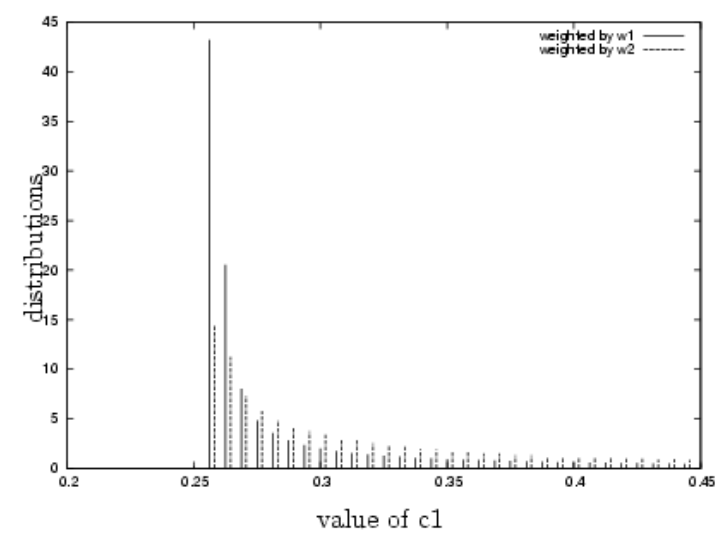

(c) order 5

(d) order 6

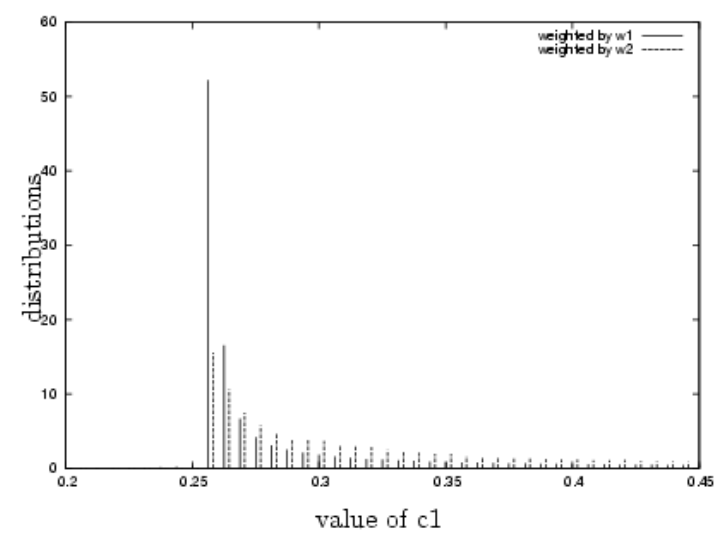

(e) order 7

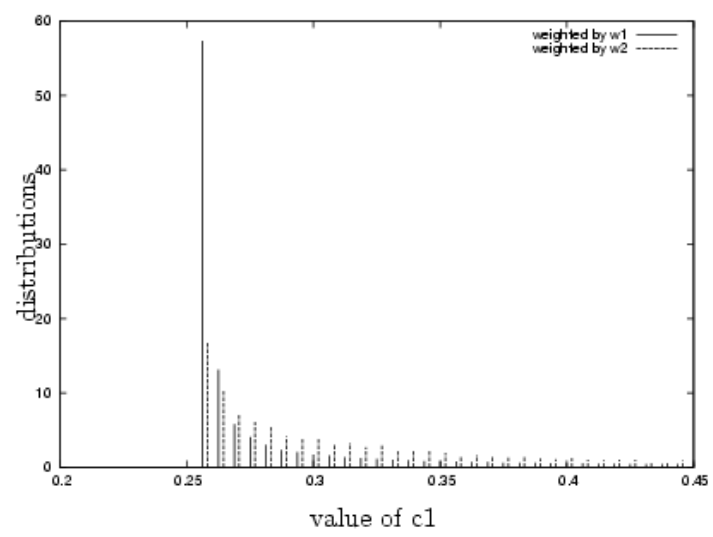

(f) order 8 
c. $c_{2}$

Region A

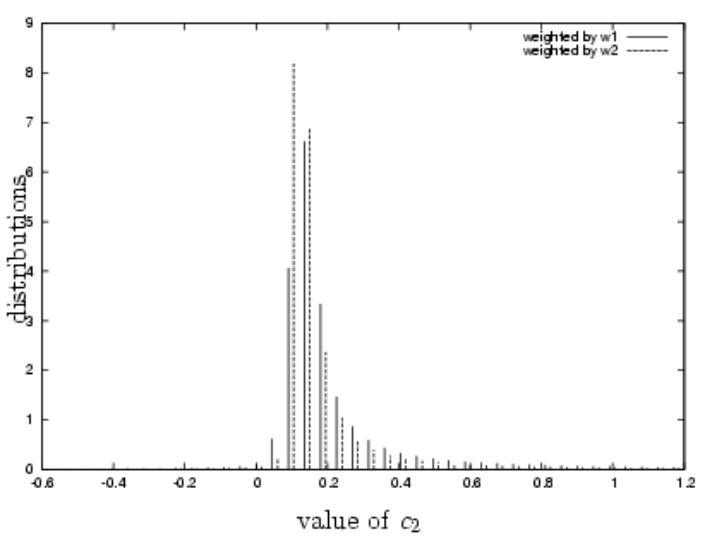

(a) order 3

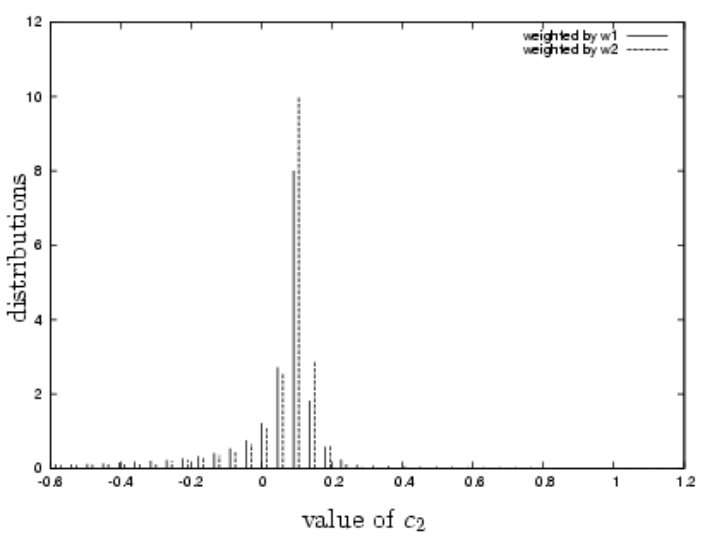

(c) order 5

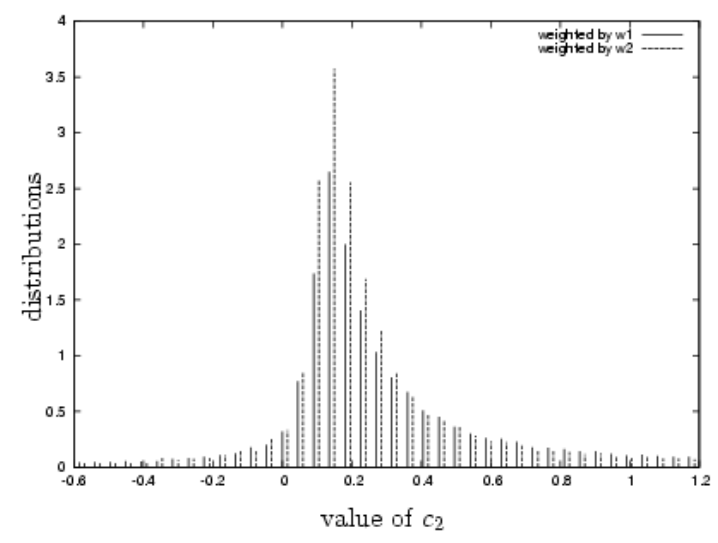

(e) order 7

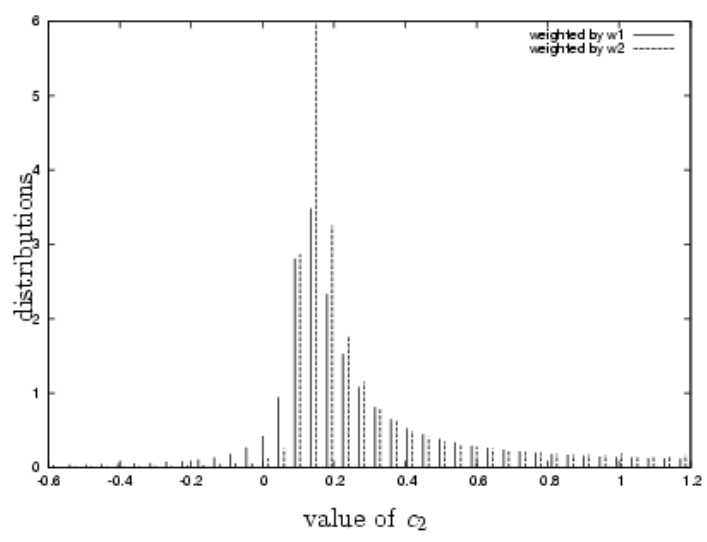

(b) order 4

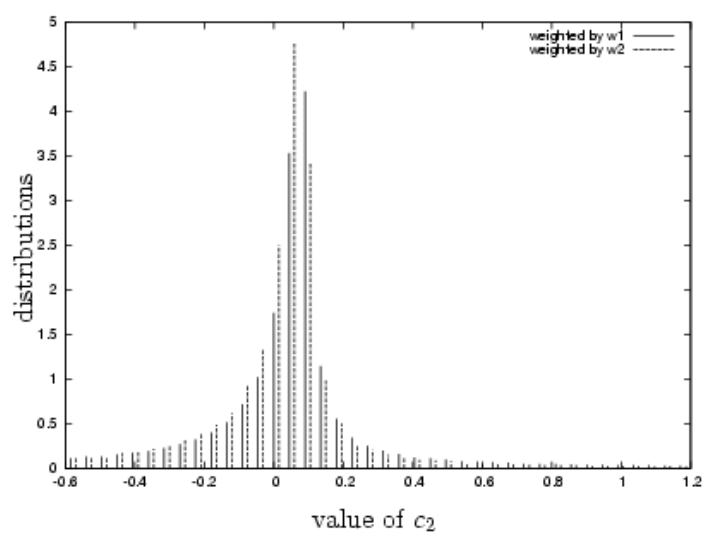

(d) order 6

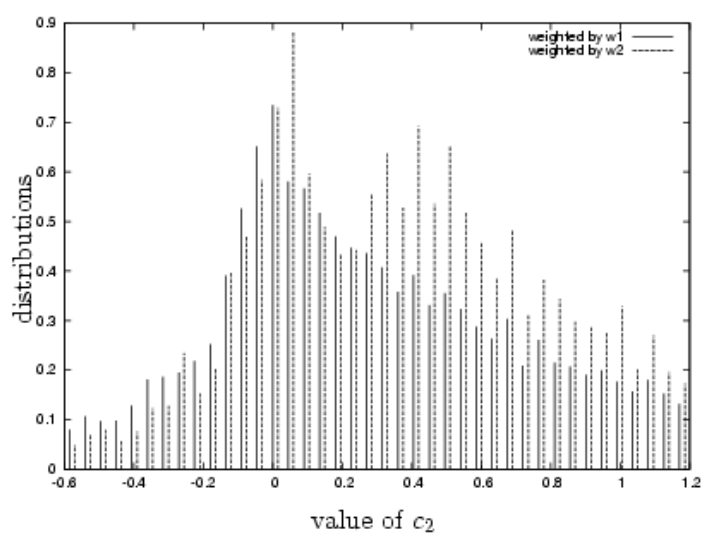

(f) order 8 


\section{Region B}
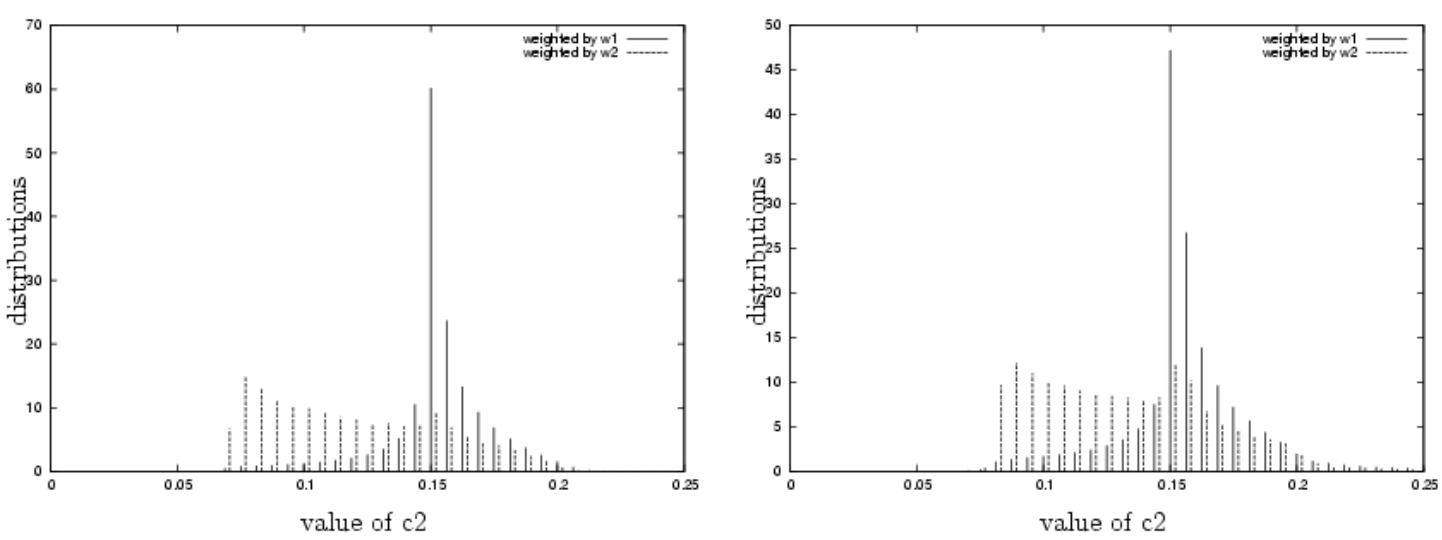

(a) order 3
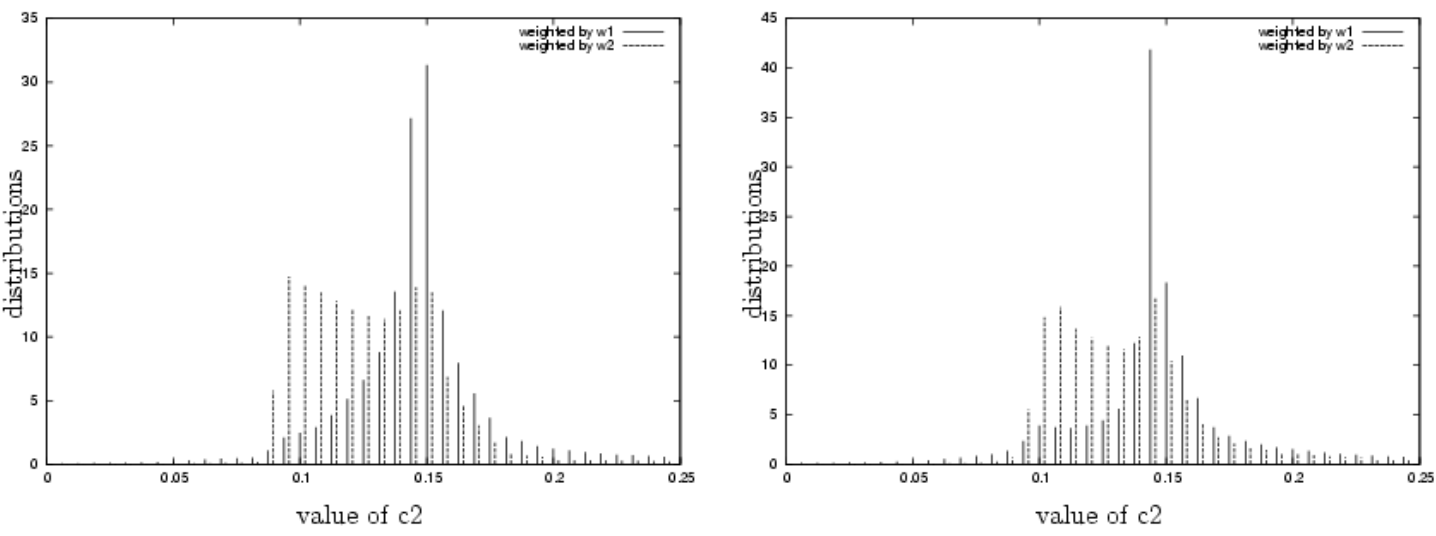

(c) order 5

(d) order 6

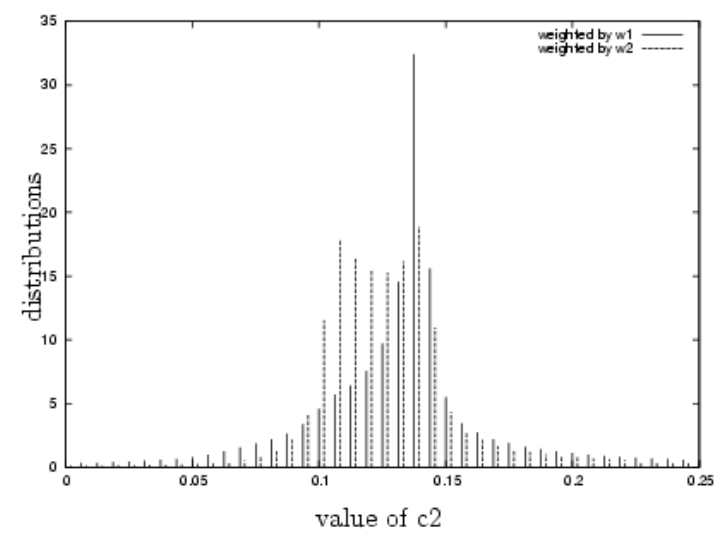

(e) order 7

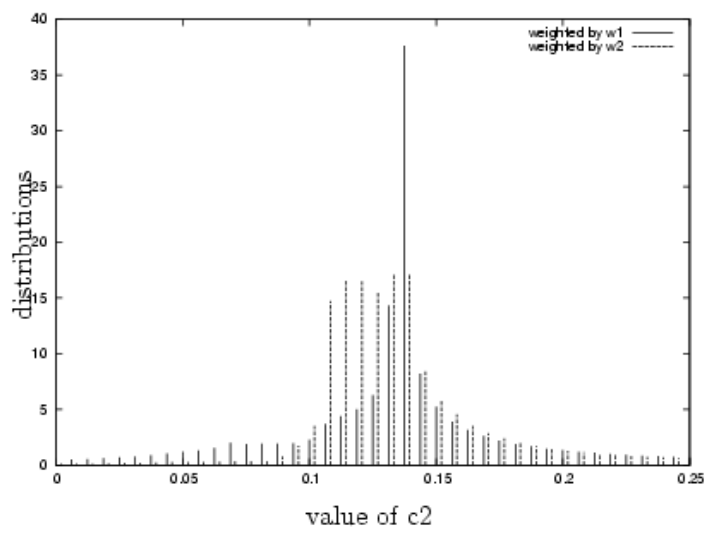

(f) order 8 


\section{Region C}
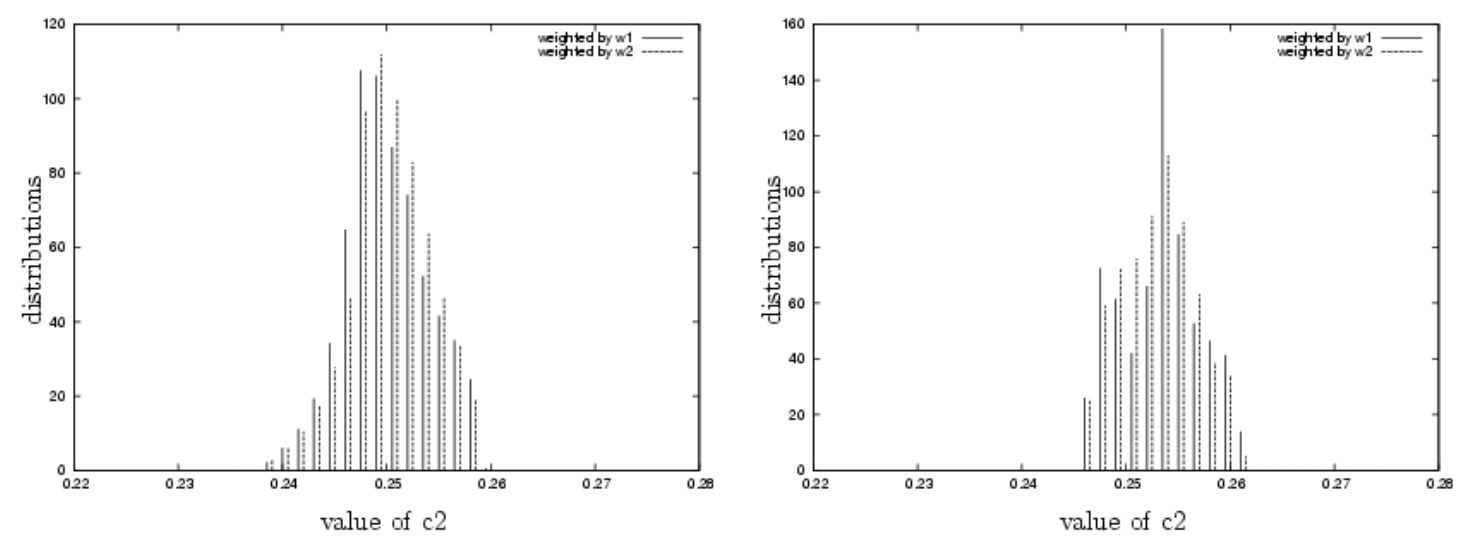

(a) order 3
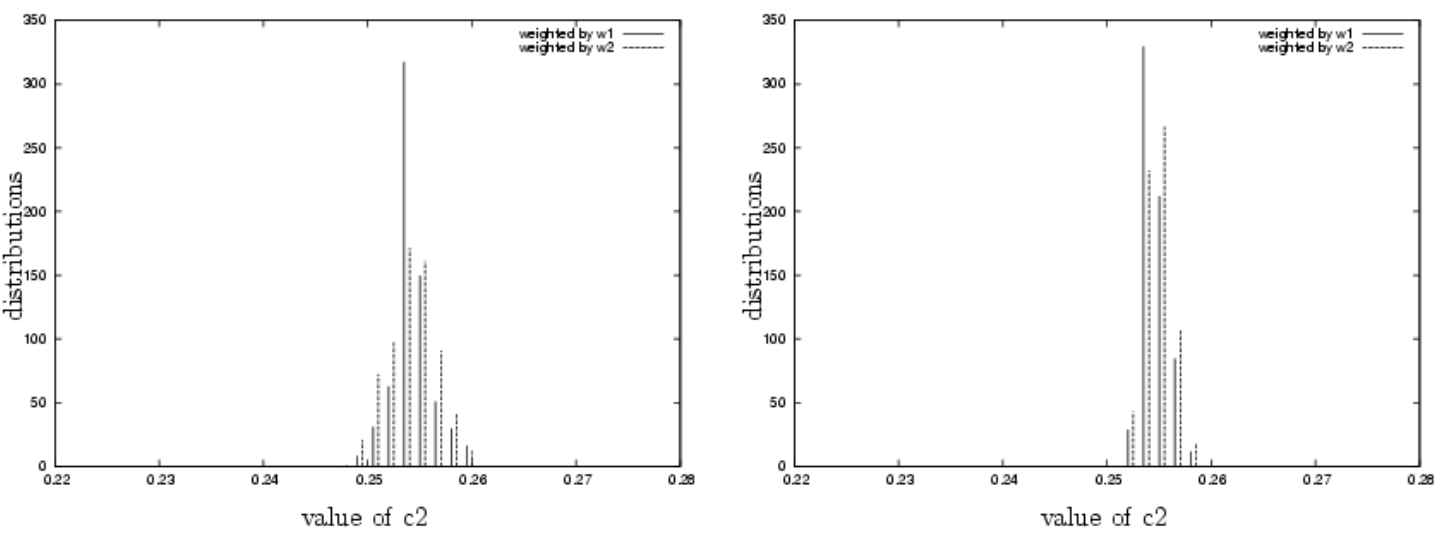

(c) order 5

(d) order 6

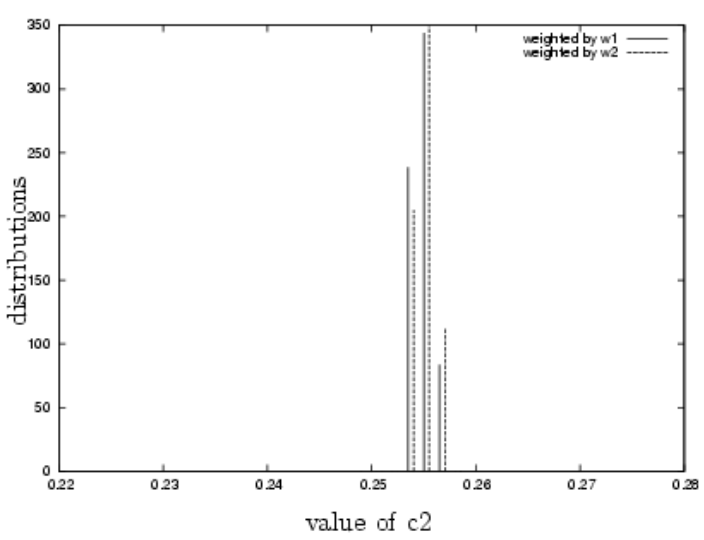

(e) order 7

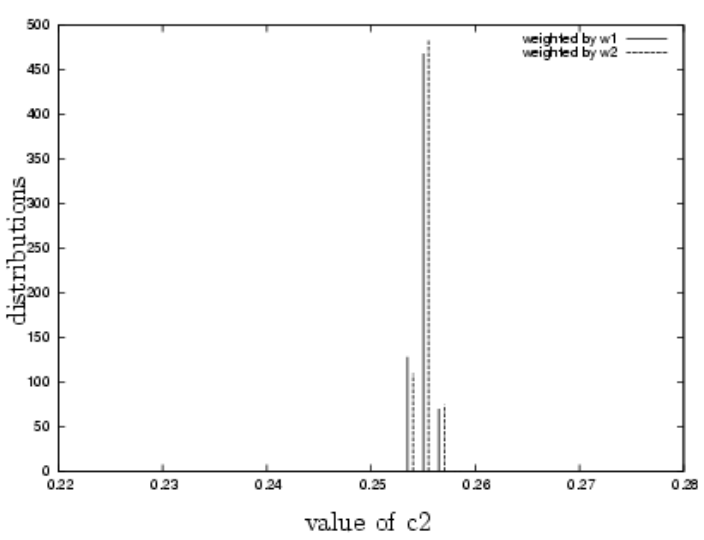

(f) order 8 


\section{Region D}
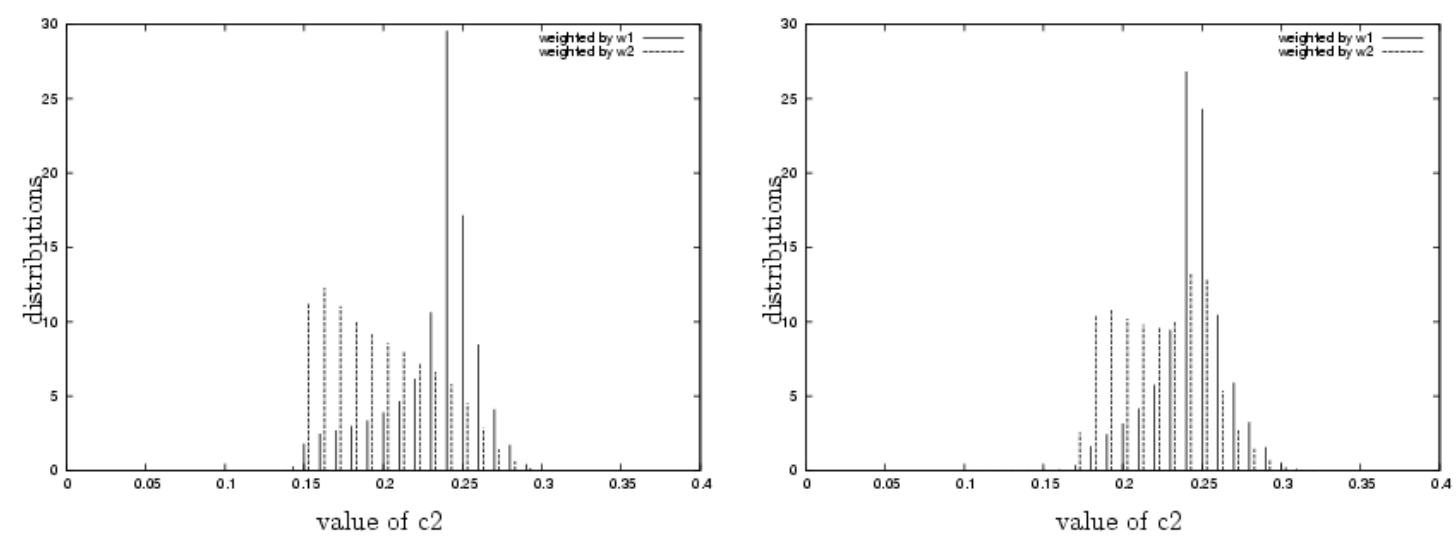

(a) order 3

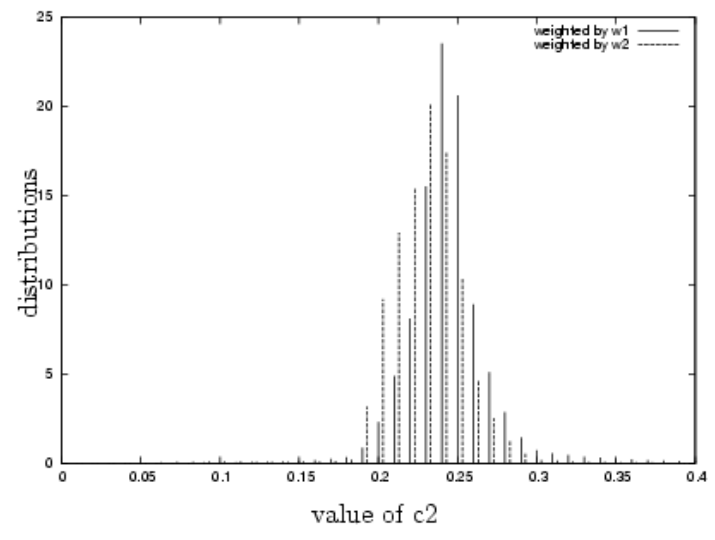

(b) order 4

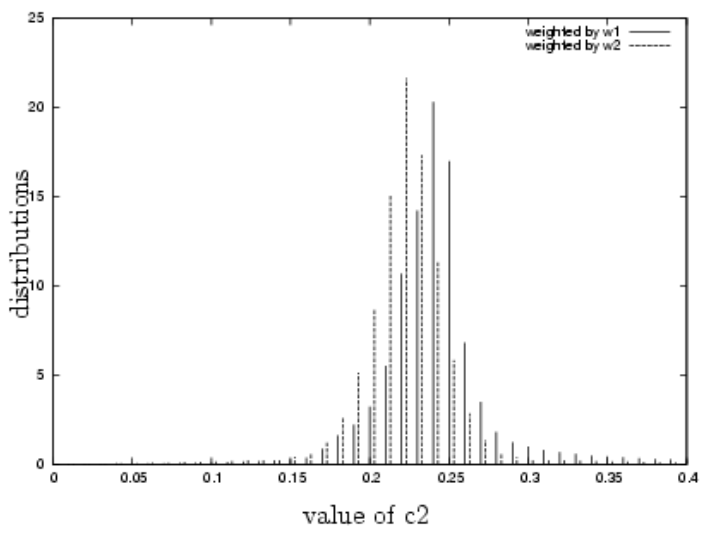

(c) order 5

(d) order 6

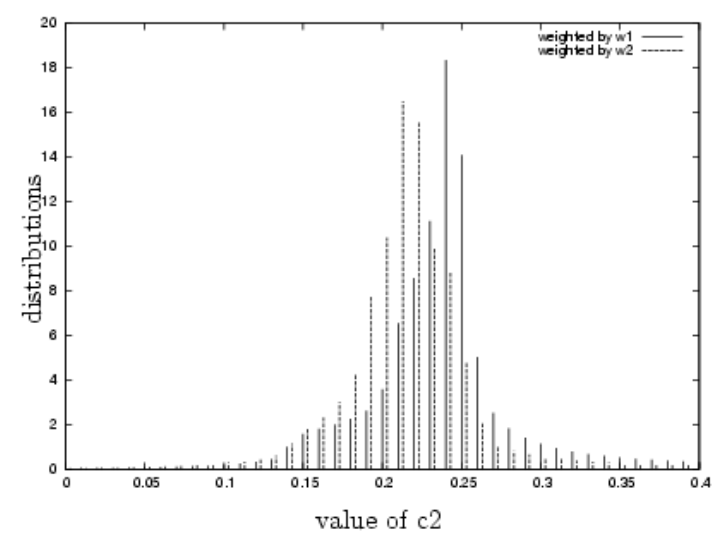

(e) order 7

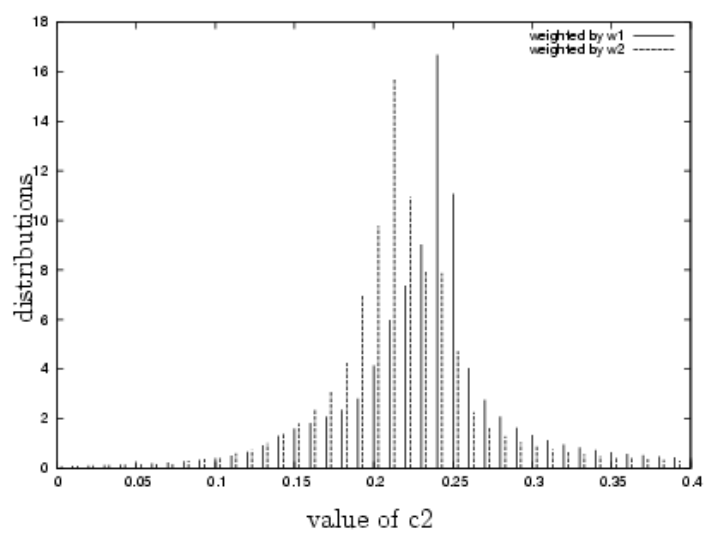

(f) order 8 


\section{2. $\mathrm{SO}(7)$ ansatz}

a. Free energy

\section{Region A}

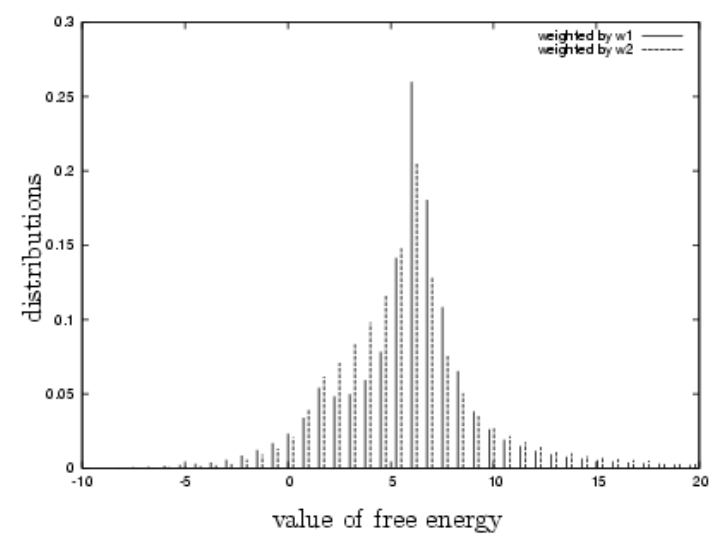

(a) order 3

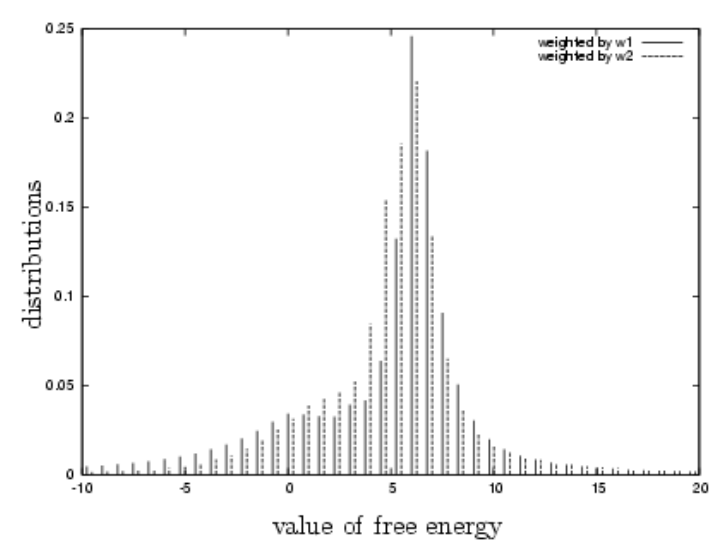

(c) order 5

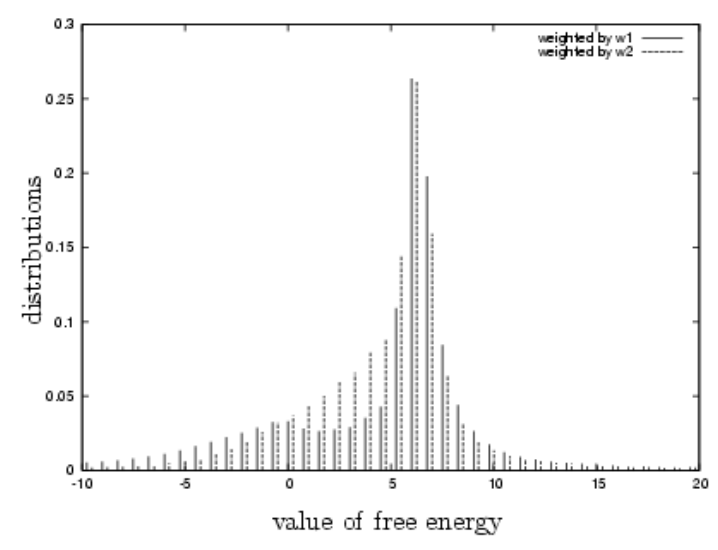

(e) order 7

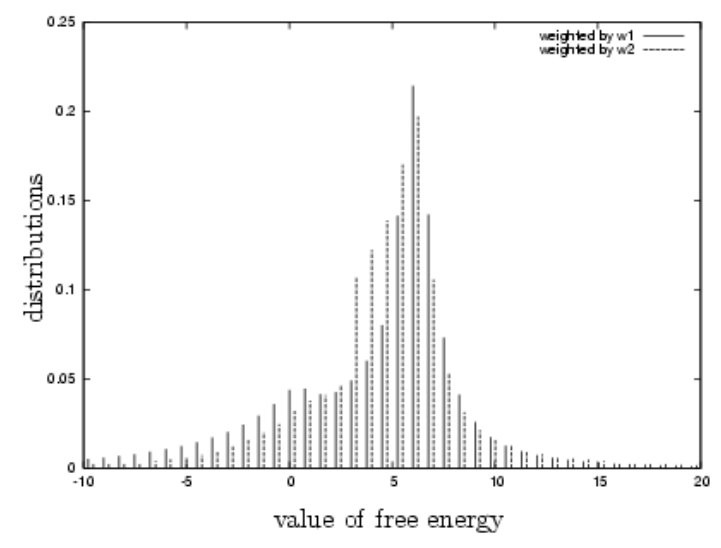

(b) order 4

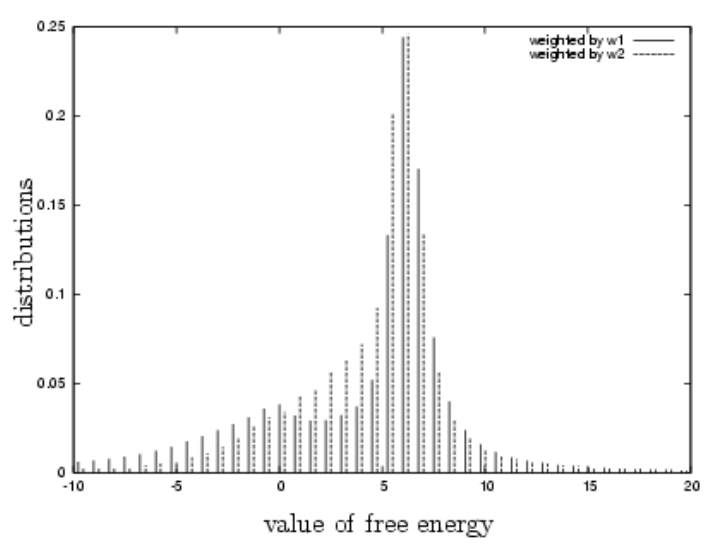

(d) order 6

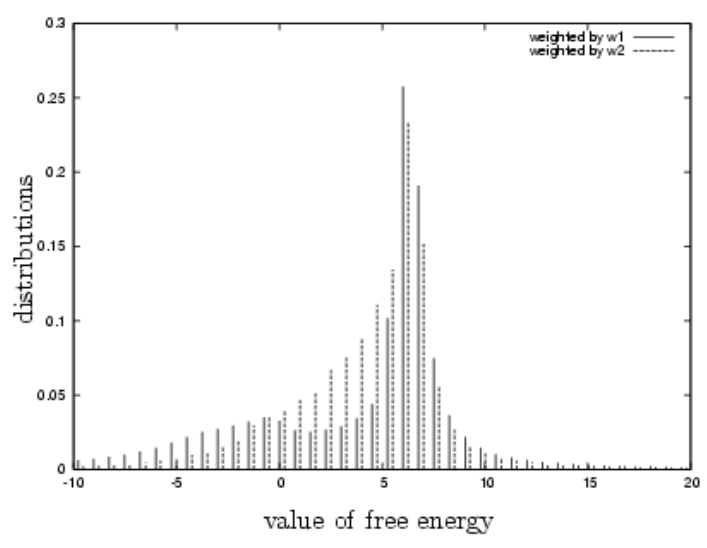

(f) order 8 


\section{Region B}

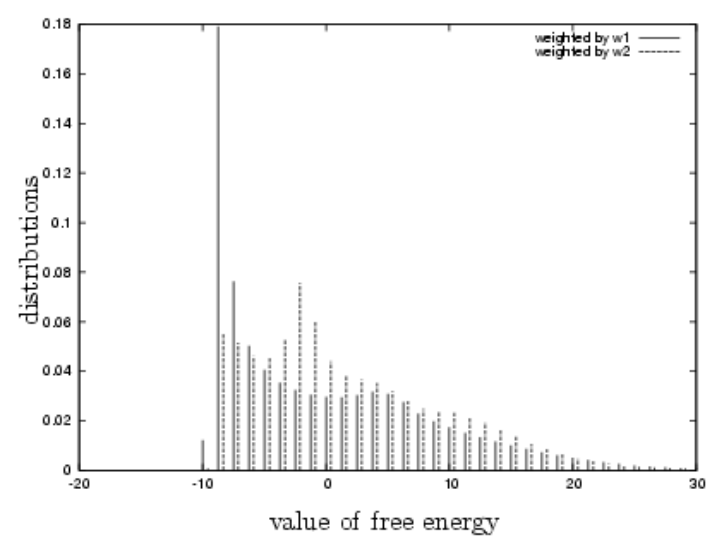

(a) order 3

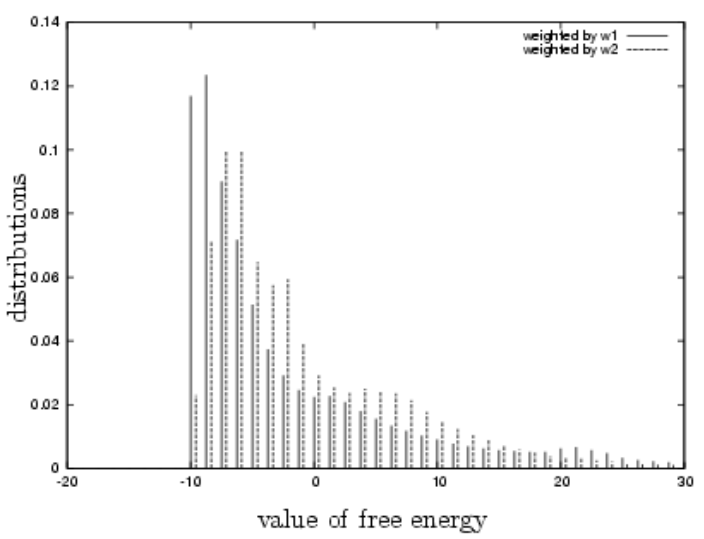

(c) order 5

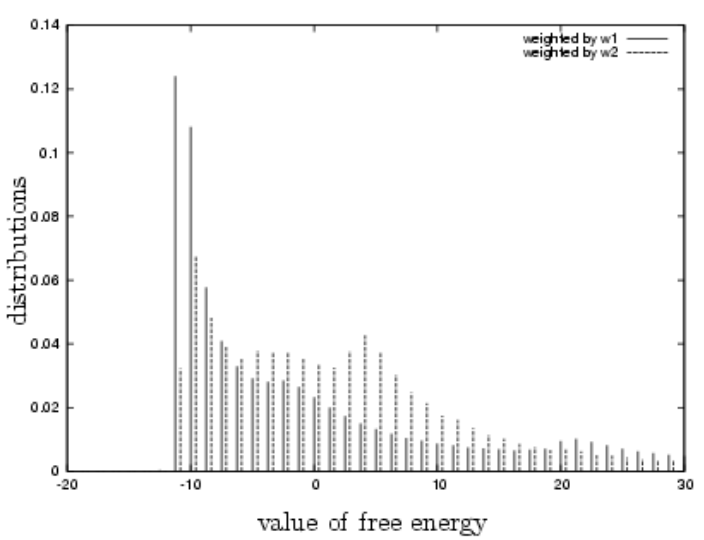

(e) order 7

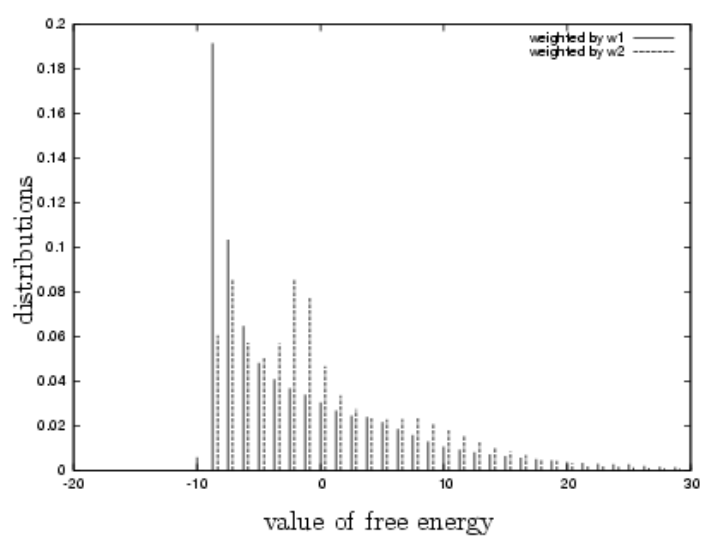

(b) order 4

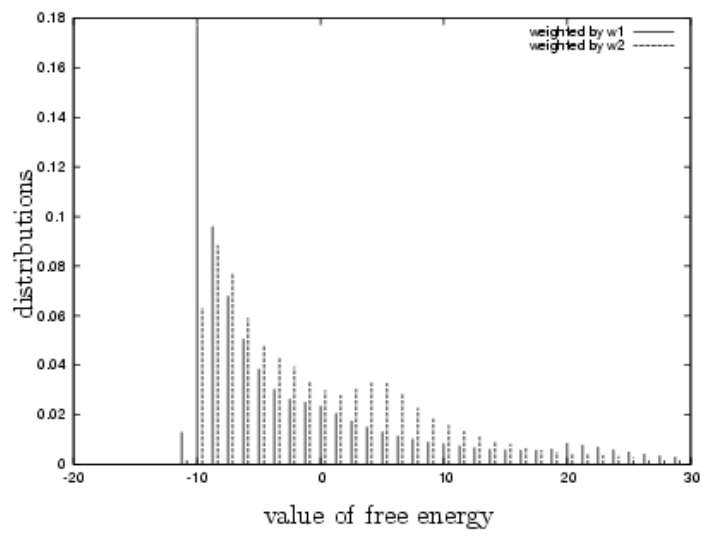

(d) order 6

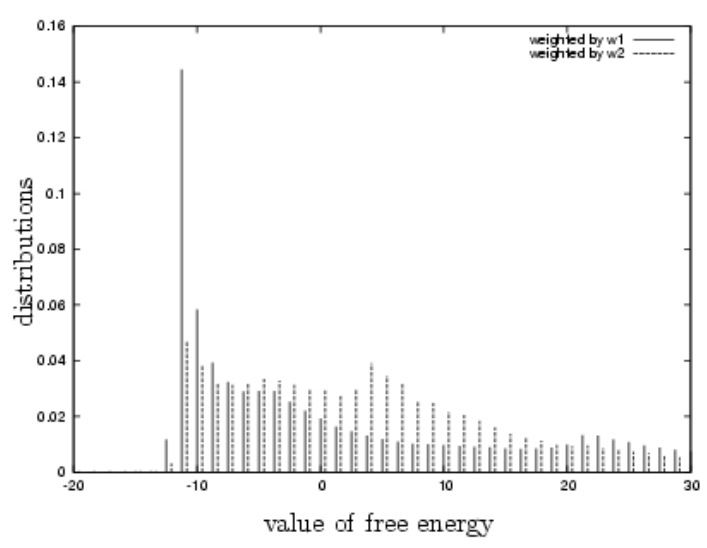

(f) order 8 
b. $\quad c_{1}$

\section{Region A}

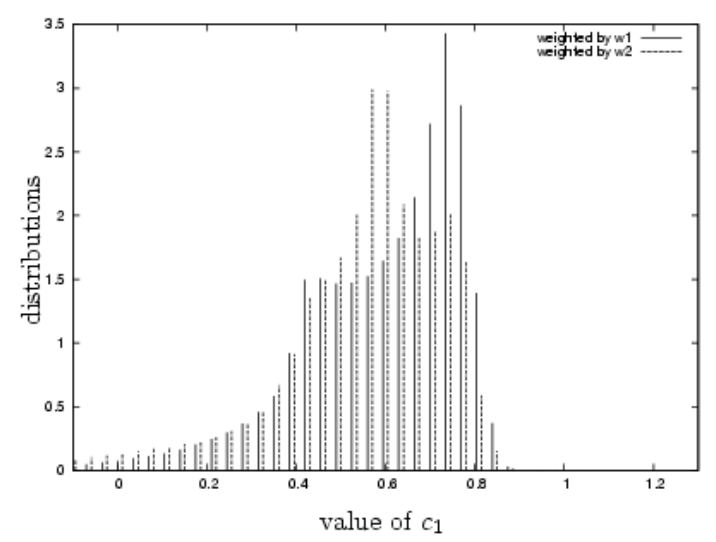

(a) order 3

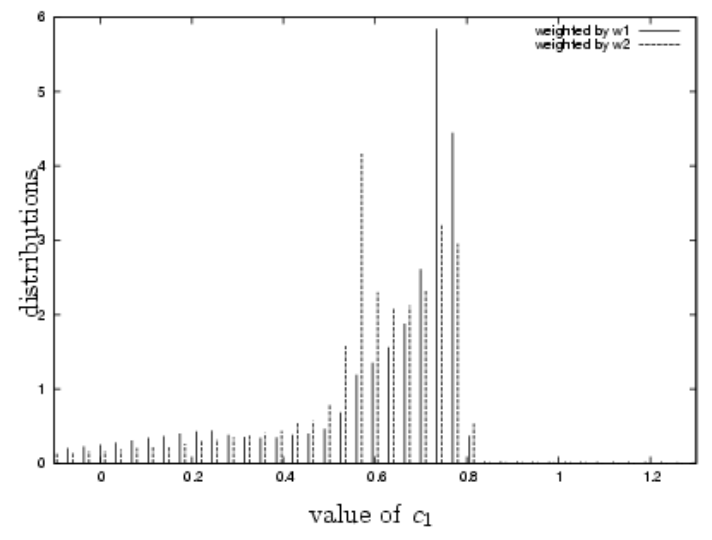

(c) order 5

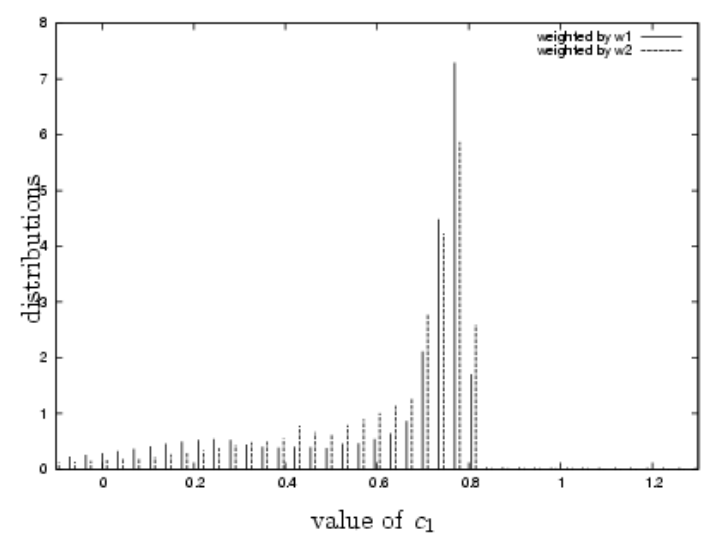

(e) order 7

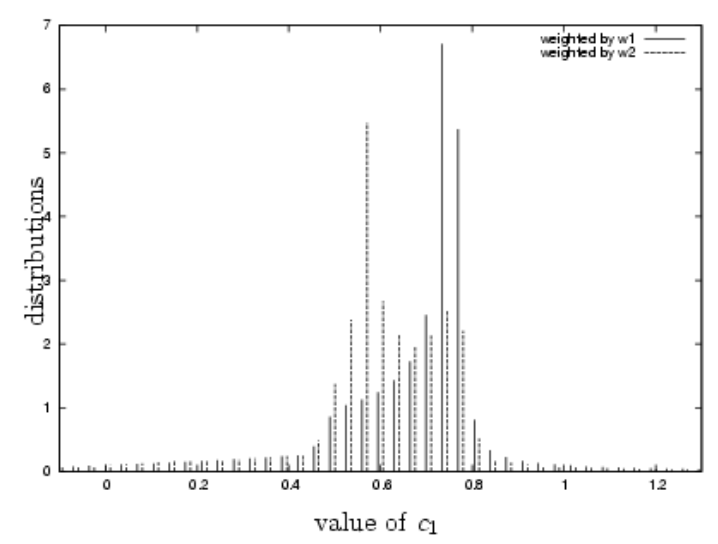

(b) order 4

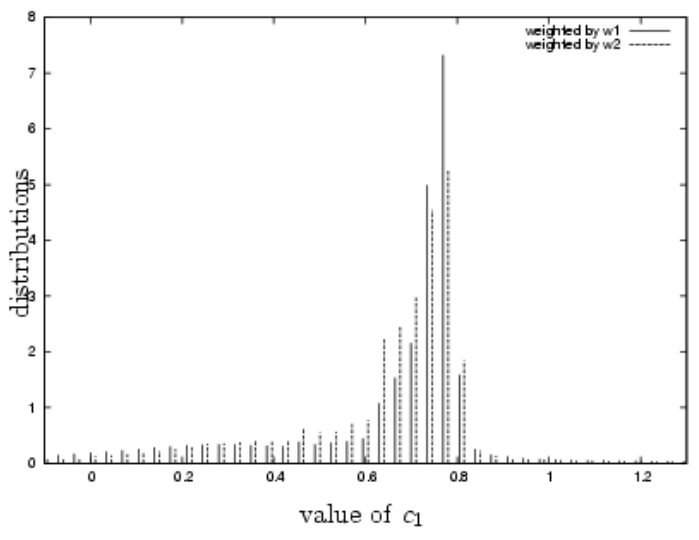

(d) order 6

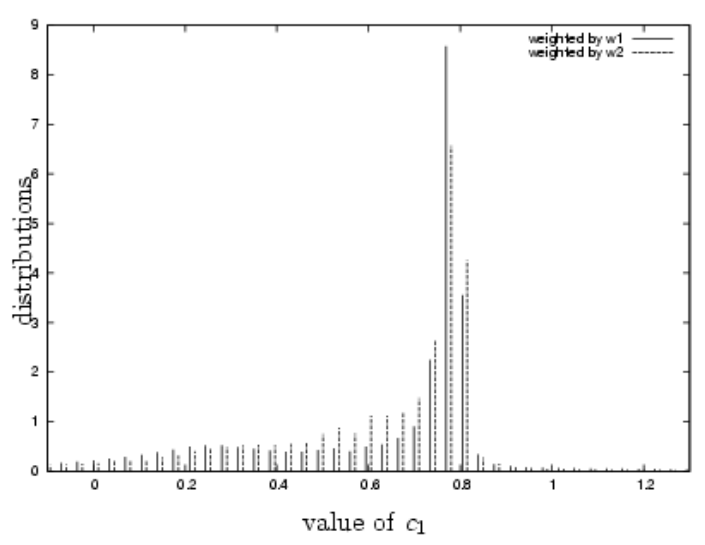

(f) order 8 


\section{Region C}
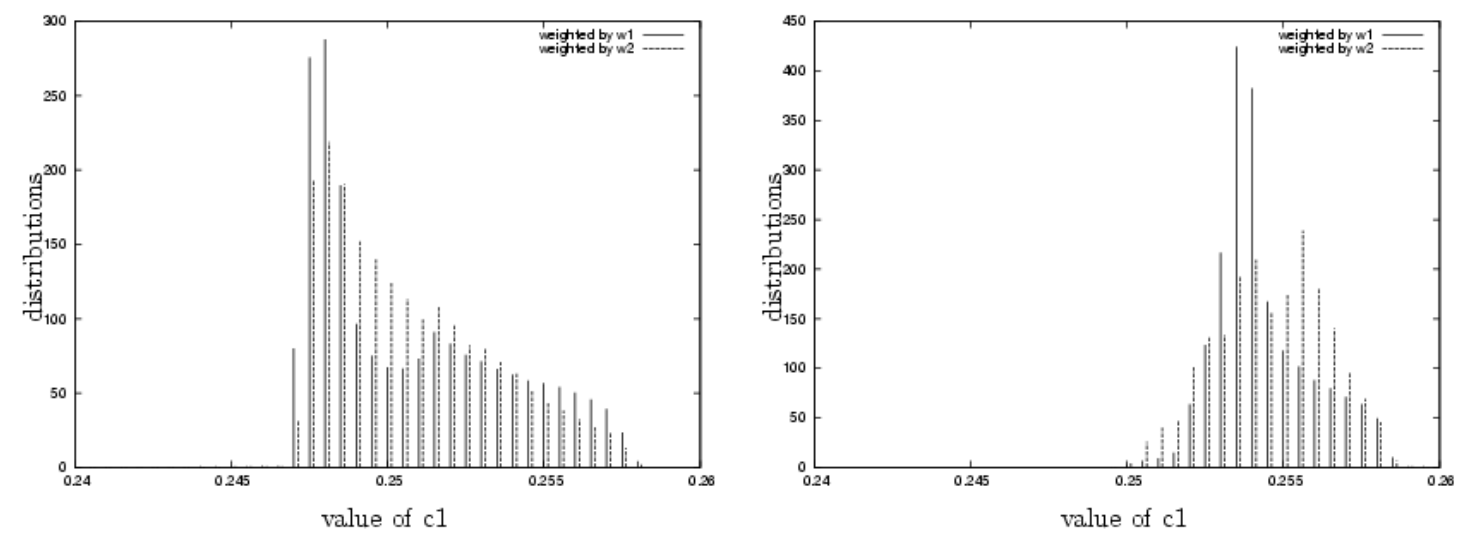

(a) order 3
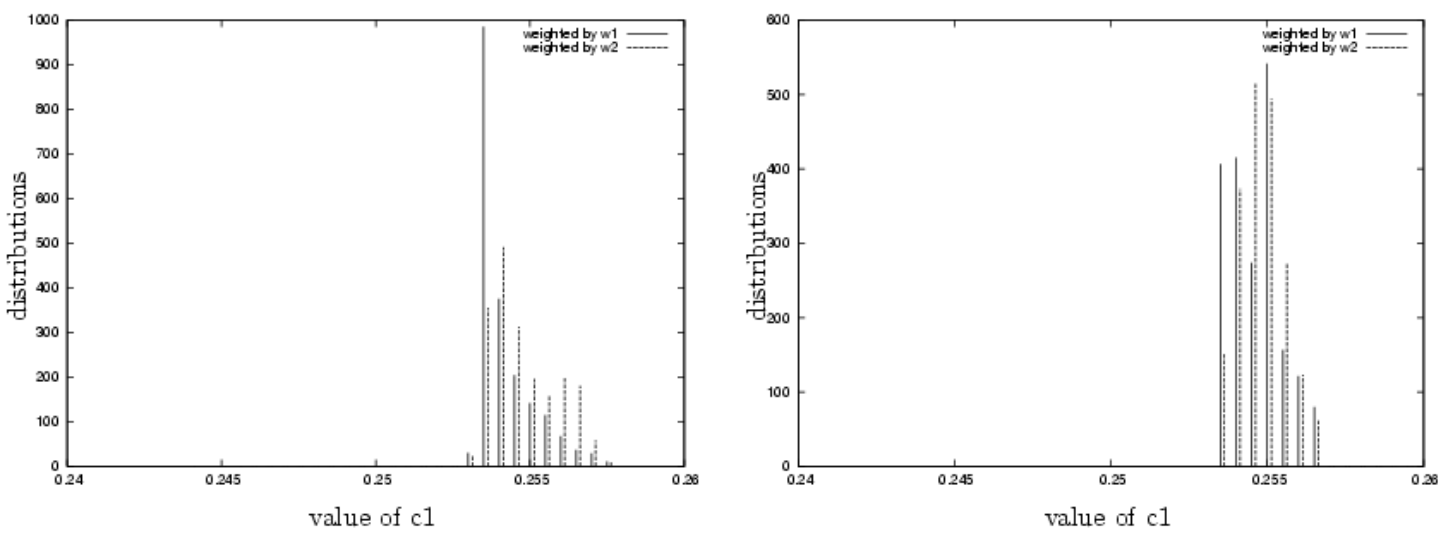

(c) order 5

(d) order 6
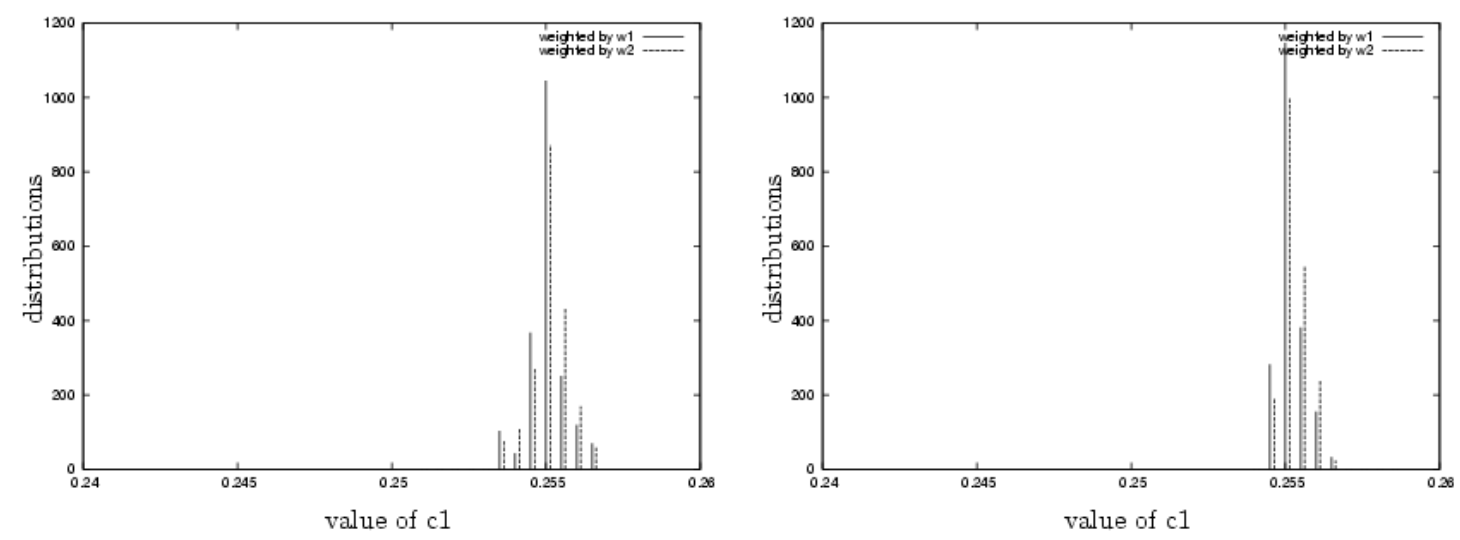

(e) order 7

(f) order 8 
c. $c_{2}$

\section{Region A}
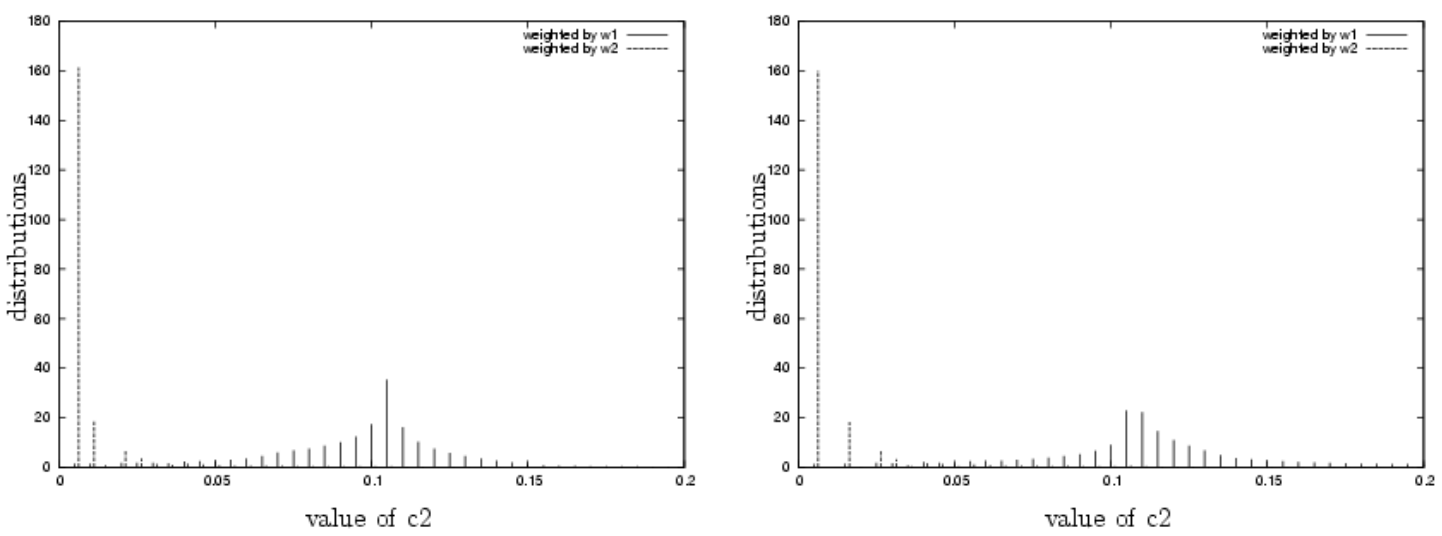

(a) order 3
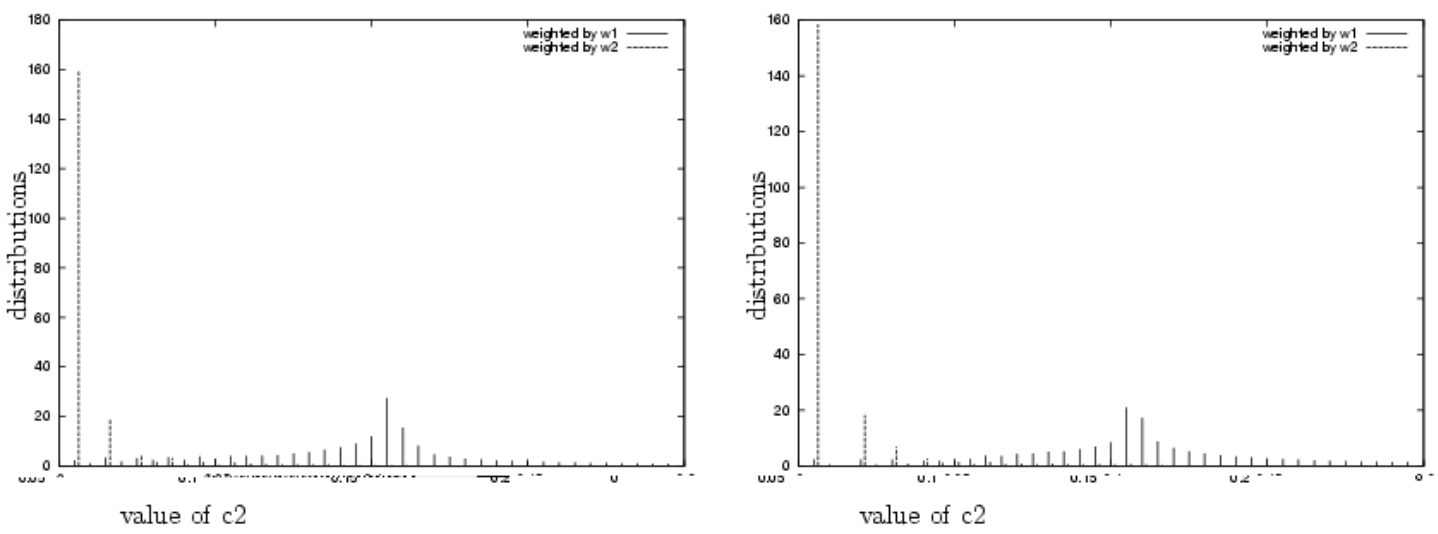

(c) order 5

(d) order 6

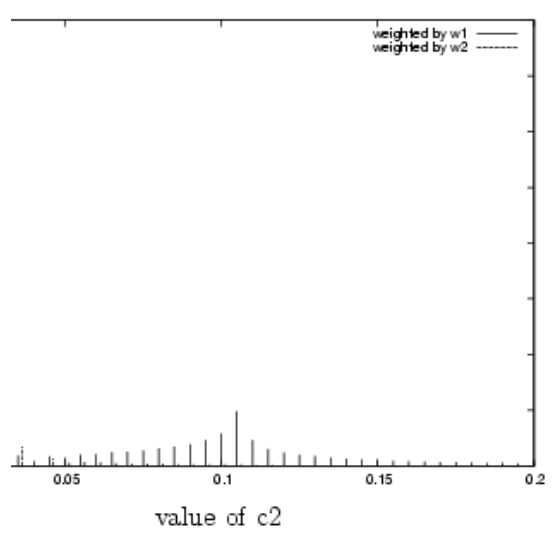

(e) order 7
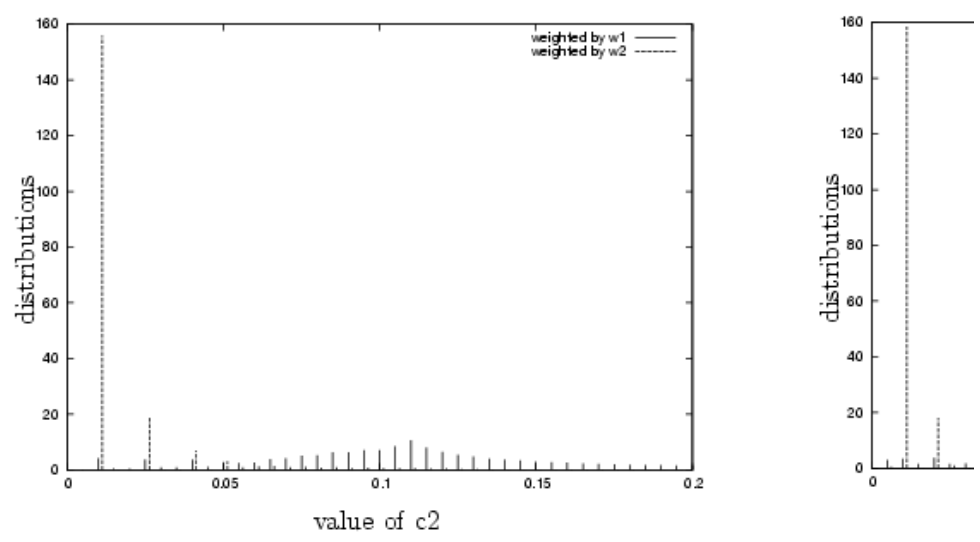

(f) order 8 
Region C
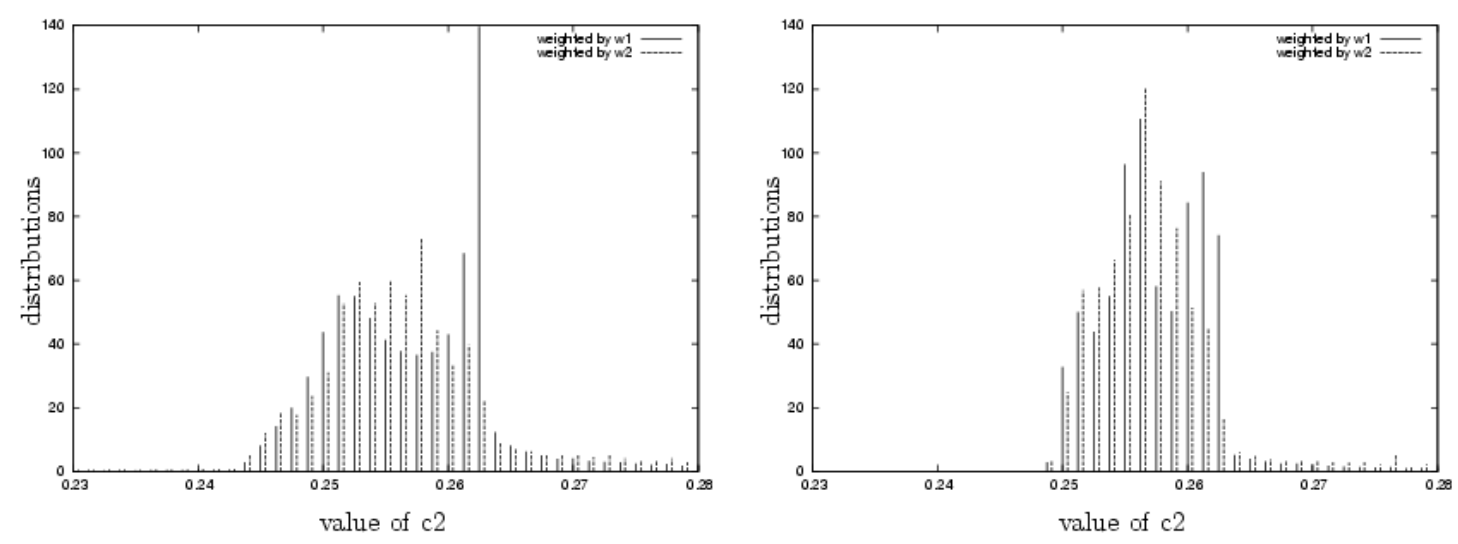

(a) order 3

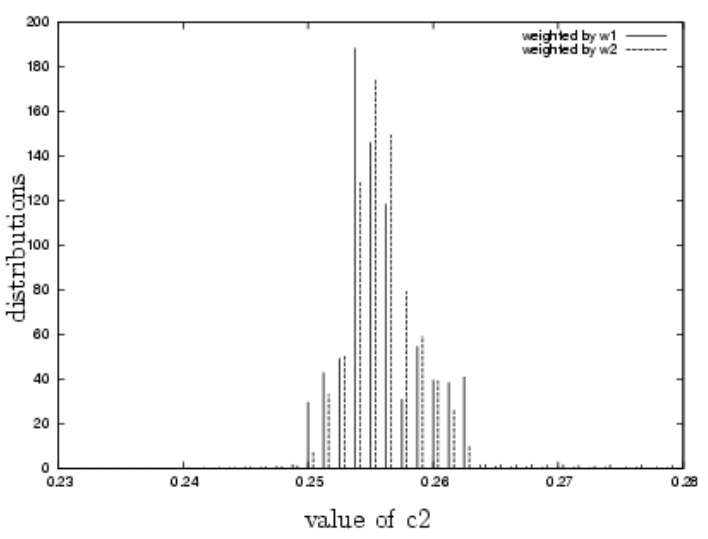

(b) order 4

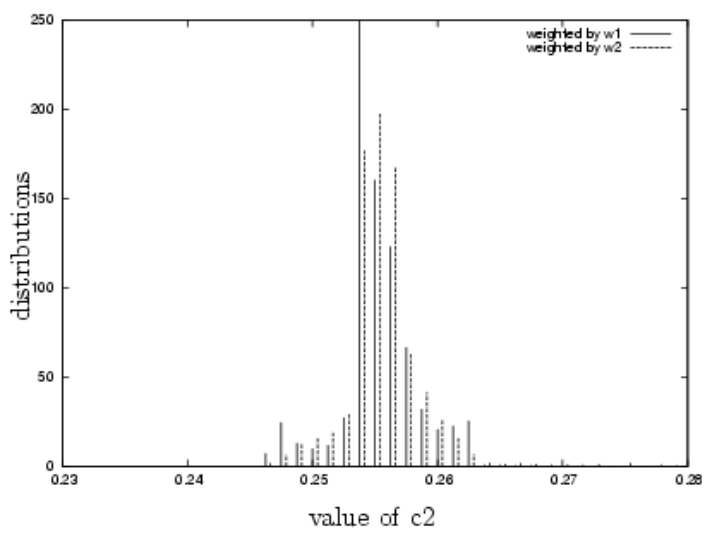

(c) order 5

(d) order 6

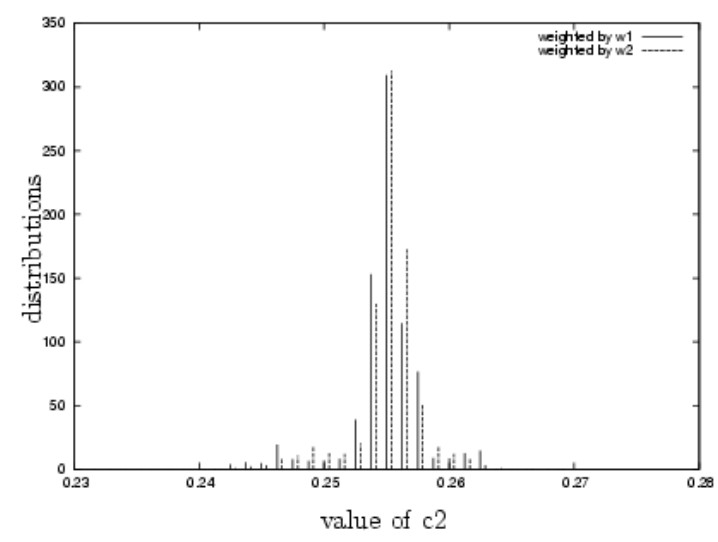

(e) order 7

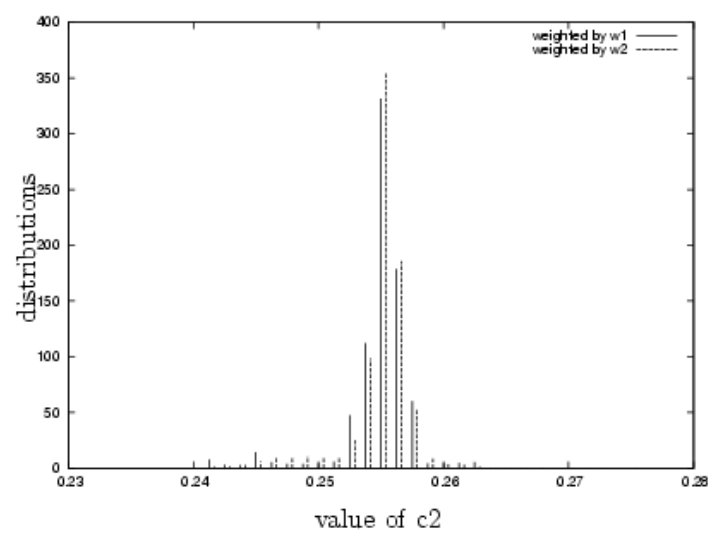

(f) order 8 


\section{3. $\mathrm{SO}(10)$-symmetric vacua}

a. Free energy

\section{Region C}
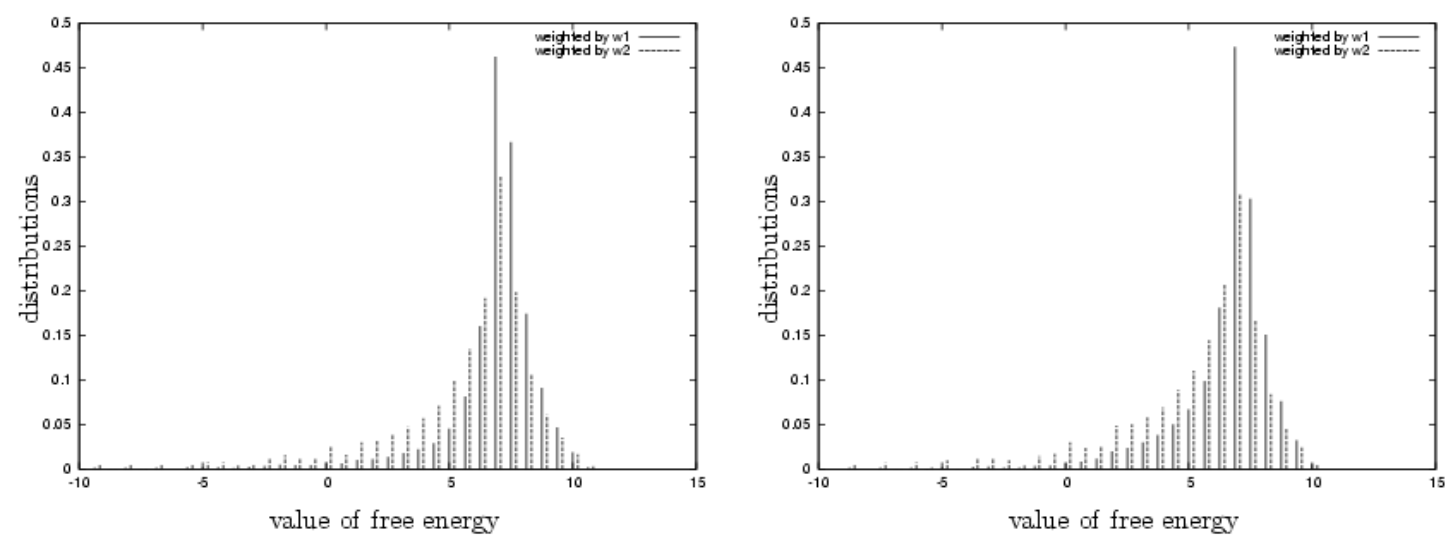

(a) order 3

(b) order 4
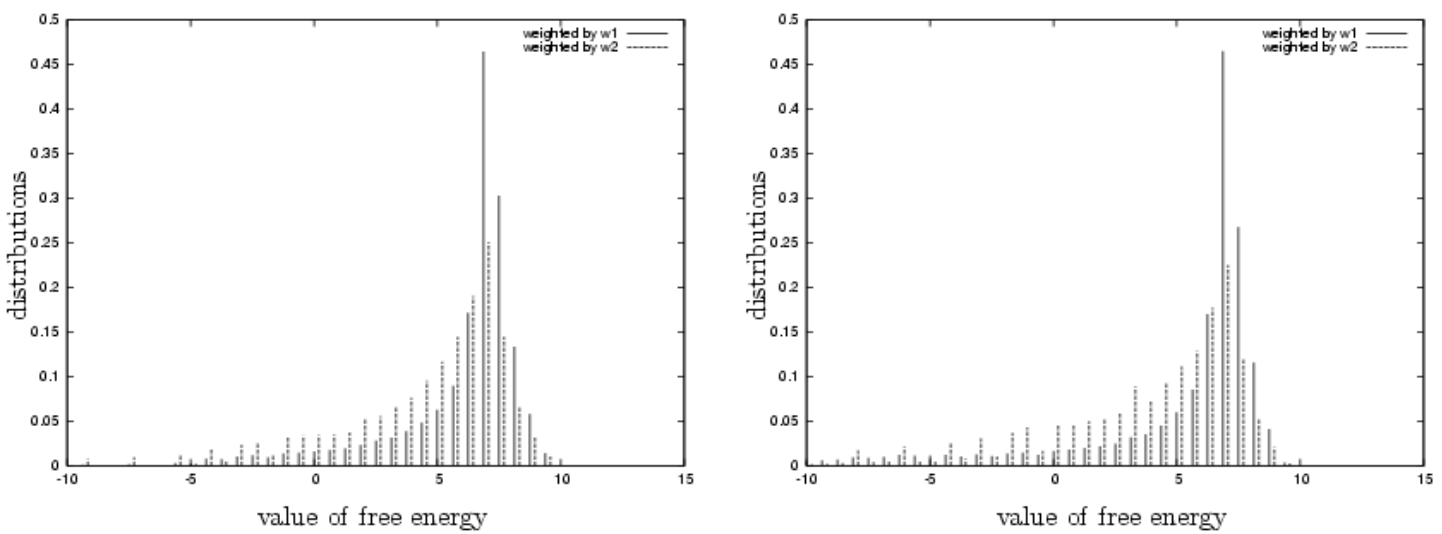

(c) order 5

(d) order 6
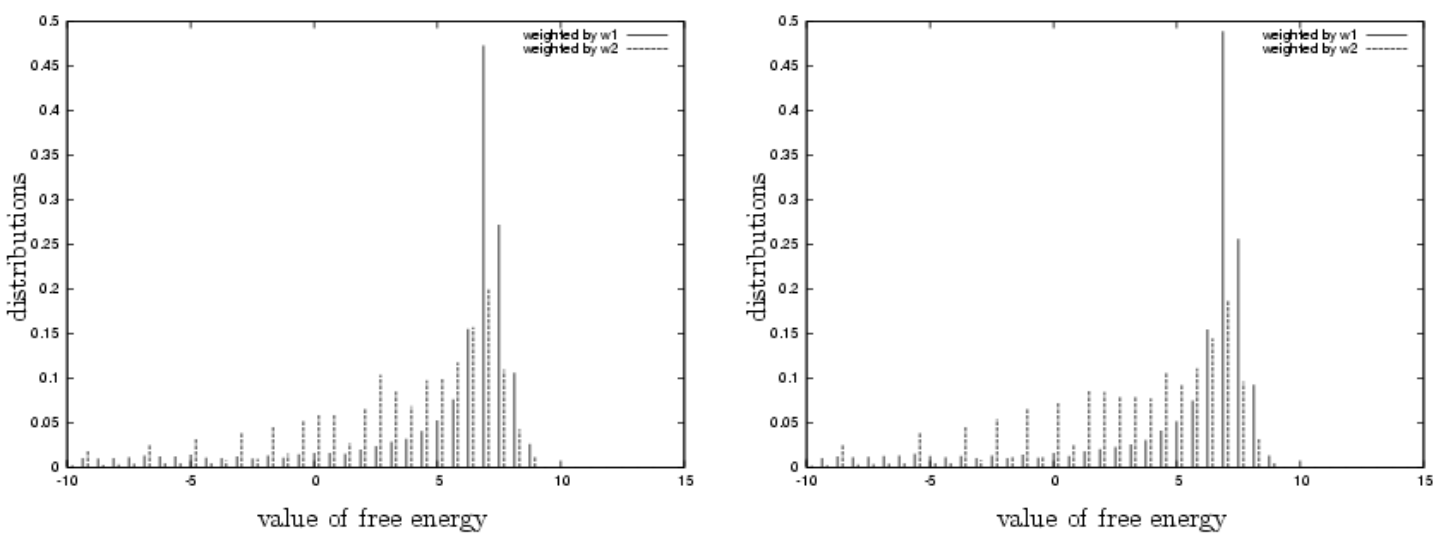

(e) order 7

(f) order 8 
[1] T. Yoneya, Prog. Theor. Phys. 51 (1974) 1907.

[2] H. Kawai, D. C. Lewellen and S. H. H. Tye, Nucl. Phys. B 288 (1987) 1.

[3] N. Ishibashi, H. Kawai, Y. Kitazawa and A. Tsuchiya, Nucl. Phys. B 498 (1997) 467.

[4] H. Aoki, S. Iso, H. Kawai, Y. Kitazawa, A. Tsuchiya and T. Tada, Prog. Theor. Phys. Suppl. 134 (1999) 47.

[5] T. Hotta, J. Nishimura and A. Tsuchiya, Nucl. Phys. B 545 (1999) 543.

[6] J. Ambjørn, K. N. Anagnostopoulos, W. Bietenholz, T. Hotta and J. Nishimura, JHEP 0007 (2000) 011.

[7] J. Ambjørn, K. N. Anagnostopoulos, W. Bietenholz, F. Hofheinz and J. Nishimura, Phys. Rev. D 65 (2002) 086001.

[8] J. Nishimura and G. Vernizzi, Phys. Rev. Lett. 85 (2000) 4664.

[9] J. Nishimura and G. Vernizzi, JHEP 0004 (2000) 015.

[10] J. Nishimura, Phys. Rev. D 65 (2002) 105012.

[11] K. N. Anagnostopoulos and J. Nishimura, Phys. Rev. D 66 (2002) 106008.

[12] J. Ambjørn, K. N. Anagnostopoulos, J. Nishimura and J. J. M. Verbaarschot, J. High Energy Phys. 0210 (2002) 062.

[13] V. I. Yukalov, Mosc. Univ. Phys. Bull. 31 (1976), 10.

[14] W. E. Caswell, Annals Phys. 123 (1979) 153.

[15] I. G. Halliday and P. Suranyi, Phys. Rev. D 21 (1980) 1529.

[16] P. M. Stevenson, Phys. Rev. D 23 (1981) 2916.

[17] J. Killingbeck, J. Phys. A 14 (1981) 1005.

[18] A. Dhar, Phys. Lett. B 128 (1983) 407.

[19] R. Guida, K. Konishi and H. Suzuki, Annals Phys. 241 (1995) 152.

[20] R. Guida, K. Konishi and H. Suzuki, Annals Phys. 249 (1996) 109.

[21] S. Kawamoto and T. Matsuo, arXiv:hep-th/0307171.

[22] T. Aoyama, T. Matsuo and Y. Shibusa, Prog. Theor. Phys. 115 (2006) 473.

[23] D. Kabat and G. Lifschytz, Nucl. Phys. B 571 (2000) 419.

[24] S. Oda and F. Sugino, JHEP 0103 (2001) 026.

[25] F. Sugino, JHEP 0107 (2001) 014. 
[26] J. Nishimura and F. Sugino, JHEP 0205 (2002) 001.

[27] H. Kawai, S. Kawamoto, T. Kuroki, T. Matsuo and S. Shinohara, Nucl. Phys. B 647 (2002) 153.

[28] H. Kawai, S. Kawamoto, T. Kuroki and S. Shinohara, Prog. Theor. Phys. 109 (2003) 115.

[29] T. Aoyama and H. Kawai, Prog. Theor. Phys. 116 (2006) 405.

[30] T. Aoyama, H. Kawai and Y. Shibusa, Prog. Theor. Phys. 115 (2006) 1179.

[31] J. Nishimura, T. Okubo and F. Sugino, Prog. Theor. Phys. 114 (2005) 487.

[32] J. Nishimura, T. Okubo and F. Sugino, JHEP 0210 (2002) 043.

[33] J. Nishimura, T. Okubo and F. Sugino, JHEP 0310 (2003) 057.

[34] In a private discussion with H. Kawai and T. Matsuo.

[35] T. Aoyama, T. Kuroki and Y. Shibusa, Phys. Rev. D 74 (2006) 106004.

[36] R. Fukuda, M. Komachiya, S. Yokojima, Y. Suzuki, K. Okumura and T. Inagaki, Prog. Theor. Phys. Suppl. 121 (1995) 1. 